Supporting Information

\title{
Construction of Quaternary Carbon Center via NHC Catalysis Initiated by an Intermolecular Heck-Type Alkyl Radical Addition
}

\author{
Lanjun Su, ${ }^{\dagger}$ Huan Sun, ${ }^{*, \dagger}$ Jikai Liu, ${ }^{*, \dagger}$ Chengming Wang ${ }^{*}, \ddagger$ \\ $\dagger$ School of Pharmaceutical Sciences \\ South-Central University for Nationalities \\ Wuhan, 430074, China \\ Email:sunh15@163.com, liujikai@mail.scuec.edu.cn \\ $\$$ Department of Chemistry, College of Chemistry and Materials Science \\ Jinan University \\ Guangzhou, 511443, China \\ Email: cmwang2019@jnu.edu.cn
}

Table of Contents:

General Methods and Materials

Conditions Screening

General Procedure for NHC-Catalyzed Radical Cyclization

General Procedure for Scalable Reaction

Characterization of Products

Diversity of the Products

Mechanism Study 


\section{General Methods and Materials:}

Unless specified, all reactions were carried out under a nitrogen atmosphere with dry solvents under anhydrous conditions. $\mathrm{N}$-arylacrylamide starting materials were synthesized according to a previous literature. ${ }^{1} \mathrm{NHCs}$ A, D-F were prepared according to literature method. ${ }^{2 a}$ NHCs I, K, L, N were purchased from Shanghai Hao Hong Pharma. NHCs B, C, M, J were purchased from Shanghai Bide Pharmatech Ltd. NHCs $\mathrm{G}-\mathrm{H}$ were synthesized according to literature method. ${ }^{2 b} \alpha$-bromo ester, $\alpha$-bromo nitrile (purity: 98\%) and 1, 4-dioxane (super dry, 99.8\%) were purchased from $J \& K$; $\mathrm{Cs}_{2} \mathrm{CO}_{3}$ (purity: 99.99\%) was purchased from Alfa Aesar; all other reagents were purchased and used without further purification unless specified otherwise. Solvents for chromatography were technical grade and distilled prior to use. Flash chromatography was performed using 200-300 mesh silica gel with the indicated solvent system according to standard techniques. Analytical thin-layer chromatography (TLC) was performed on pre-coated, glass-backed silica gel plates. Visualization of the developed chromatogram was performed by UV absorbance (254 $\mathrm{nm})$. The heat source was used Heating Block for Cylindrical Vial, which purchased from Tianjin Boost Tech. Co., Ltd. ${ }^{1} \mathrm{H}$ NMR and ${ }^{13} \mathrm{C}$ NMR data were recorded on Bruker $300 \mathrm{M}$ nuclear resonance spectrometers unless otherwise specified, respectively.

Chemical shifts $(\delta)$ in ppm are reported as quoted relative to the residual signals of chloroform ( ${ }^{1} \mathrm{H} 7.26 \mathrm{ppm}$ or $\left.{ }^{13} \mathrm{C} 77.00 \mathrm{ppm}\right)$. Multiplicities are described as: s (singlet), bs (broad singlet), d (doublet), t (triplet), q (quartet), $m$ (multiplet); and coupling constants $(J)$ are reported in Hertz $(\mathrm{Hz}) .{ }^{13} \mathrm{C}$ NMR spectra were recorded with total proton decoupling. High resolution mass spectrometry (HRMS) analysis was performed using electrospray ionization (ESI) with a quadrupole-time of flight (QTOF) mass analyzer. HRMS (ESI) analysis was performed by The Analytical Instrumentation Center at College of Chemistry and Materials Science, Jinan University, and (HRMS) data were reported with ion mass/charge $(\mathrm{m} / \mathrm{z})$ ratios as values in atomic mass units. 


\section{Conditions Screening a}

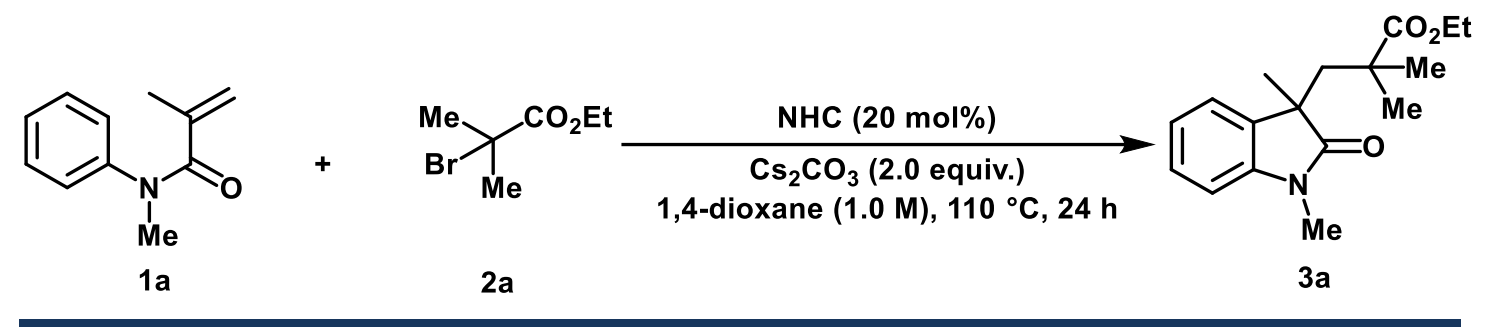

\begin{tabular}{llll}
\hline Entry Solvent & Catalyst & Base & Yield
\end{tabular}

\begin{tabular}{|c|c|c|c|c|}
\hline 1 & THF & $\mathrm{NHCA}$ & $\mathrm{Cs}_{2} \mathrm{CO}_{3}$ & $59 \%$ \\
\hline 2 & 1, 4-dioxane & NHC A & $\mathrm{Cs}_{2} \mathrm{CO}_{3}$ & $86 \%$ \\
\hline 3 & $\mathrm{MeCN}$ & $\mathrm{NHCA}$ & $\mathrm{Cs}_{2} \mathrm{CO}_{3}$ & $67 \%$ \\
\hline 4 & Toluene & $\mathrm{NHCA}$ & $\mathrm{Cs}_{2} \mathrm{CO}_{3}$ & $15 \%$ \\
\hline 5 & DCE & $\mathrm{NHCA}$ & $\mathrm{Cs}_{2} \mathrm{CO}_{3}$ & $26 \%$ \\
\hline 6 & DMF & $\mathrm{NHCA}$ & $\mathrm{Cs}_{2} \mathrm{CO}_{3}$ & $60 \%$ \\
\hline 7 & DBE & $\mathrm{NHCA}$ & $\mathrm{Cs}_{2} \mathrm{CO}_{3}$ & $43 \%$ \\
\hline 8 & DMSO & $\mathrm{NHCA}$ & $\mathrm{Cs}_{2} \mathrm{CO}_{3}$ & $14 \%$ \\
\hline $9^{b}$ & 1, 4-dioxane & $\mathrm{NHCA}$ & $\mathrm{Cs}_{2} \mathrm{CO}_{3}$ & $0 \%$ \\
\hline $10^{c}$ & 1, 4-dioxane & $\mathrm{NHCA}$ & $\mathrm{Cs}_{2} \mathrm{CO}_{3}$ & $8 \%$ \\
\hline $11^{d}$ & 1, 4-dioxane & $\mathrm{NHCA}$ & $\mathrm{Cs}_{2} \mathrm{CO}_{3}$ & $17 \%$ \\
\hline $12^{\mathrm{e}}$ & 1, 4-dioxane & $\mathrm{NHCA}$ & $\mathrm{Cs}_{2} \mathrm{CO}_{3}$ & $30 \%$ \\
\hline $13^{f}$ & 1, 4-dioxane & $\mathrm{NHCA}$ & $\mathrm{Cs}_{2} \mathrm{CO}_{3}$ & $61 \%$ \\
\hline 14 & 1, 4-dioxane $(0.1 \mathrm{M})$ & $\mathrm{NHCA}$ & $\mathrm{Cs}_{2} \mathrm{CO}_{3}$ & $17 \%$ \\
\hline 15 & 1, 4-dioxane $(0.2 \mathrm{M})$ & $\mathrm{NHCA}$ & $\mathrm{Cs}_{2} \mathrm{CO}_{3}$ & $26 \%$ \\
\hline 16 & 1, 4-dioxane (0.3 M) & $\mathrm{NHCA}$ & $\mathrm{Cs}_{2} \mathrm{CO}_{3}$ & $39 \%$ \\
\hline 17 & 1, 4-dioxane (0.5 M) & $\mathrm{NHCA}$ & $\mathrm{Cs}_{2} \mathrm{CO}_{3}$ & $40 \%$ \\
\hline 18 & 1, 4-dioxane & $\mathrm{NHCA}$ & $\mathrm{Cs}_{2} \mathrm{CO}_{3}$ (0.5 equiv.) & $54 \%$ \\
\hline 19 & 1, 4-dioxane & $\mathrm{NHCA}$ & $\mathrm{Cs}_{2} \mathrm{CO}_{3}$ (1.0 equiv.) & $64 \%$ \\
\hline 20 & 1, 4-dioxane & $\mathrm{NHCA}$ & $\mathrm{Cs}_{2} \mathrm{CO}_{3}$ (1.2 equiv.) & $59 \%$ \\
\hline 21 & 1, 4-dioxane & $\mathrm{NHCA}$ & $\mathrm{Cs}_{2} \mathrm{CO}_{3}$ (1.5 equiv.) & $77 \%$ \\
\hline 22 & 1, 4-dioxane & $\mathrm{NHCA}$ & $\mathrm{Cs}_{2} \mathrm{CO}_{3}$ (2.5 equiv.) & $46 \%$ \\
\hline 23 & 1, 4-dioxane & $\mathrm{NHC} \mathrm{A}$ & $\mathrm{Na}_{2} \mathrm{CO}_{3}$ & $37 \%$ \\
\hline 24 & 1, 4-dioxane & $\mathrm{NHC} \mathrm{A}$ & DIPEA & $53 \%$ \\
\hline 25 & 1, 4-dioxane & $\mathrm{NHCA}$ & $\mathrm{NaHCO}_{3}$ & $45 \%$ \\
\hline 26 & 1, 4-dioxane & $\mathrm{NHCA}$ & $t$-BuOK & $38 \%$ \\
\hline 27 & 1, 4-dioxane & $\mathrm{NHC} \mathrm{A}$ & $\mathrm{NaOAc}$ & $16 \%$ \\
\hline 28 & 1, 4-dioxane & $\mathrm{NHCA}$ & $\mathrm{NaOH}$ & $19 \%$ \\
\hline 29 & 1, 4-dioxane & $\mathrm{NHCA}$ & DBU & $9 \%$ \\
\hline 30 & 1, 4-dioxane & $\mathrm{NHCA}$ & $\mathrm{Et}_{3} \mathrm{~N}$ & $34 \%$ \\
\hline 31 & 1, 4-dioxane & $\mathrm{NHC} \mathrm{B}$ & $\mathrm{Cs}_{2} \mathrm{CO}_{3}$ & $0 \%$ \\
\hline 32 & 1, 4-dioxane & $\mathrm{NHCC}$ & $\mathrm{Cs}_{2} \mathrm{CO}_{3}$ & $<5 \%$ \\
\hline 33 & 1, 4-dioxane & NHC D & $\mathrm{Cs}_{2} \mathrm{CO}_{3}$ & $<5 \%$ \\
\hline
\end{tabular}




\begin{tabular}{lllll}
34 & 1, 4-dioxane & $\mathrm{NHC} \mathrm{E}$ & $\mathrm{Cs}_{2} \mathrm{CO}_{3}$ & $49 \%$ \\
35 & 1, 4-dioxane & $\mathrm{NHC} \mathrm{F}$ & $\mathrm{Cs}_{2} \mathrm{CO}_{3}$ & $56 \%$ \\
36 & 1, 4-dioxane & $\mathrm{NHC} \mathrm{G}$ & $\mathrm{Cs}_{2} \mathrm{CO}_{3}$ & $54 \%$ \\
37 & 1, 4-dioxane & $\mathrm{NHC} \mathrm{H}$ & $\mathrm{Cs}_{2} \mathrm{CO}_{3}$ & $31 \%$ \\
38 & 1,4 -dioxane & $\mathrm{NHC} \mathrm{I}$ & $\mathrm{Cs}_{2} \mathrm{CO}_{3}$ & $<5 \%$ \\
39 & 1,4 -dioxane & $\mathrm{NHC} \mathrm{J}$ & $\mathrm{Cs}_{2} \mathrm{CO}_{3}$ & $<5 \%$ \\
40 & 1,4 -dioxane & $\mathrm{NHC} \mathrm{K}$ & $\mathrm{Cs}_{2} \mathrm{CO}_{3}$ & $20 \%$ \\
41 & 1, 4-dioxane & $\mathrm{NHC} \mathrm{L}$ & $\mathrm{Cs}_{2} \mathrm{CO}_{3}$ & $<5 \%$ \\
42 & 1,4 -dioxane & $\mathrm{NHC} \mathrm{M}$ & $\mathrm{Cs}_{2} \mathrm{CO}_{3}$ & $<5 \%$ \\
43 & 1,4 -dioxane & $\mathrm{NHC} \mathrm{N}$ & $\mathrm{Cs}_{2} \mathrm{CO}_{3}$ & $36 \%$ \\
\hline
\end{tabular}

${ }^{a}$ Reaction on a $0.25 \mathrm{mmol}$ scale, using 1a (1.0 quiv.), 2a (1.5 equiv.), NHC (20 mol\%), base (2.0 equiv.), solvent $(0.25 \mathrm{~mL}), 110{ }^{\circ} \mathrm{C}$, under $\mathrm{N}_{2}, 24 \mathrm{~h},{ }^{1} \mathrm{H}$ NMR yield; ${ }^{b} \mathrm{rt} ;{ }^{c} 40{ }^{\circ} \mathrm{C} ;{ }^{d} 60{ }^{\circ} \mathrm{C}$; ${ }^{e} 80^{\circ} \mathrm{C} ;{ }^{f} 100$ ${ }^{\circ} \mathrm{C}$.

\section{Note:}

$\mathrm{DCE}=1$, 2-dichloroethane; $\mathrm{DBE}=$ Dibutyl ether; DIPEA = N, N-diisopropylethylamine; $\mathrm{DBU}=1$, 8-Diazabicyclo[5, 4, 0]undec-7-ene.

For entries 38-43, chiral NHCs were used. However, no ee values of $3 a$ were detected.

The structure of NHC catalysts:

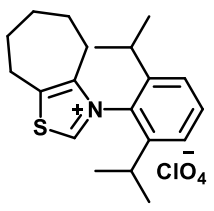

NHC A

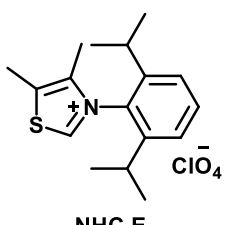

NHC E

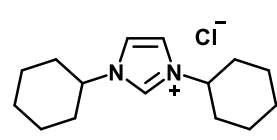

NHC B

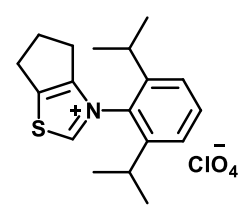

NHC F

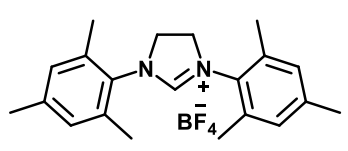

NHC C

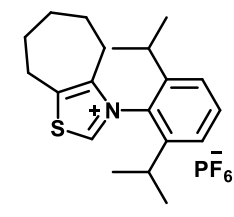

NHC G

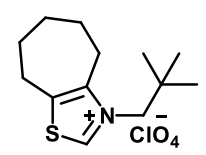

NHC D

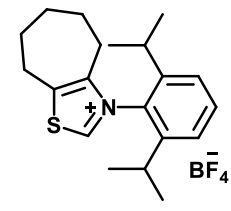

NHC H<smiles></smiles>

NHC I

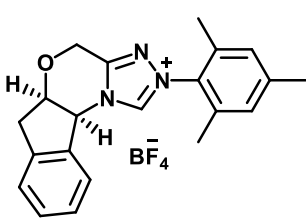

NHC J

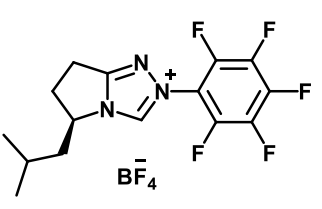

NHC K

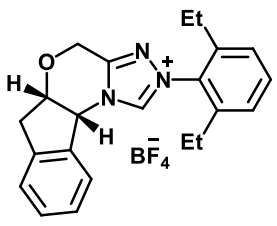

NHC L

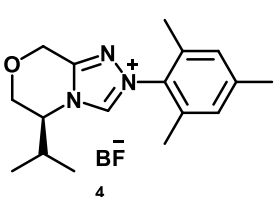

NHC M

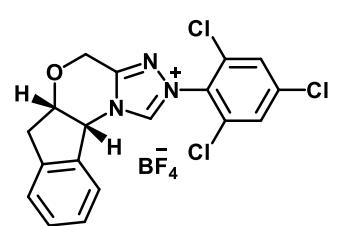

NHC N 


\section{General Procedure for NHC-Catalyzed Radical Cyclization}

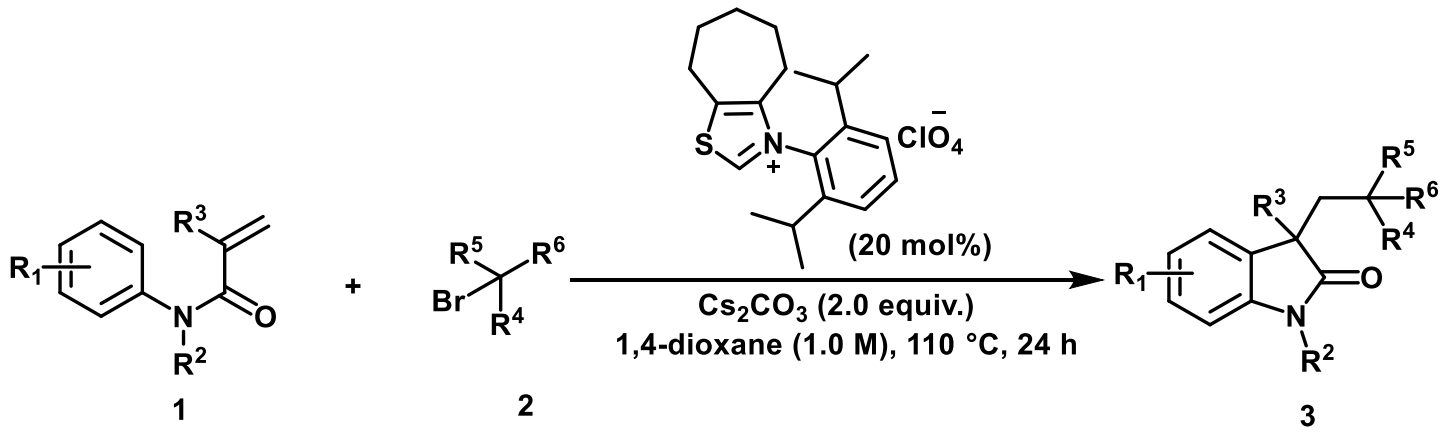

General procedure A: $N$-arylacrylamide 1 ( $0.3 \mathrm{mmol}, 1.0$ equiv.), NHC A (20 mol\% ) and $\mathrm{Cs}_{2} \mathrm{CO}_{3}(0.6 \mathrm{mmol}, 2.0$ equiv.) were weighed into a Schlenk tube. The reaction vessel was capped and subjected to three vacuum-purge/nitrogen-flush cycles. Then 2 ( $0.45 \mathrm{mmol}, 1.5$ equiv.) dissolved in 1, 4-dioxane $(0.3 \mathrm{~mL})$ was added through the side-arm by syringe. The reaction was stirred under nitrogen at $110^{\circ} \mathrm{C}$ for $24 \mathrm{~h}$. Upon complete consumption of $\mathrm{N}$-arylacrylamide 1 compound, the reaction was cooled to room temperature. Volatile solvent and reagents were removed by rotary evaporation and the residue was purified by silica gel flash chromatography using petroleum ether/EtOAc (50:1 to 10:1) to afford the desired product 3 .

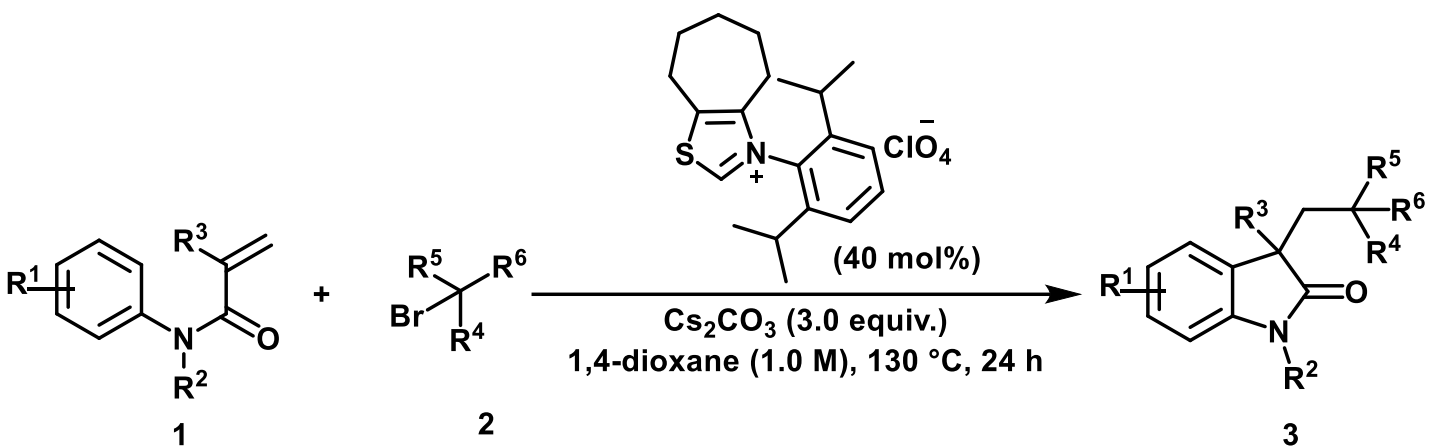

General procedure B: N-arylacrylamide 1 ( $0.3 \mathrm{mmol}, 1.0$ equiv.), NHC A (40 mol\% ) and $\mathrm{Cs}_{2} \mathrm{CO}_{3}(0.9 \mathrm{mmol}, 3.0$ equiv.) were weighed into a Schlenk tube. The reaction vessel was capped and subjected to three vacuum-purge/nitrogen-flush cycles. Then 2 (0.9 mmol, 3.0 equiv.) dissolved in 1, 4-dioxane $(0.3 \mathrm{~mL})$ was added through the side-arm by syringe. The reaction was stirred under nitrogen at $130^{\circ} \mathrm{C}$ for $24 \mathrm{~h}$. Upon complete consumption of $\mathrm{N}$-arylacrylamide 1 compound, the reaction was cooled to room temperature. Volatile solvent and reagents were removed by rotary evaporation and the residue was purified by silica gel flash chromatography using petroleum ether/EtOAc (50:1 to 10:1) to afford the desired product 3. 


\section{General Procedure for Scalable Reaction (6.0 mmol scale)}

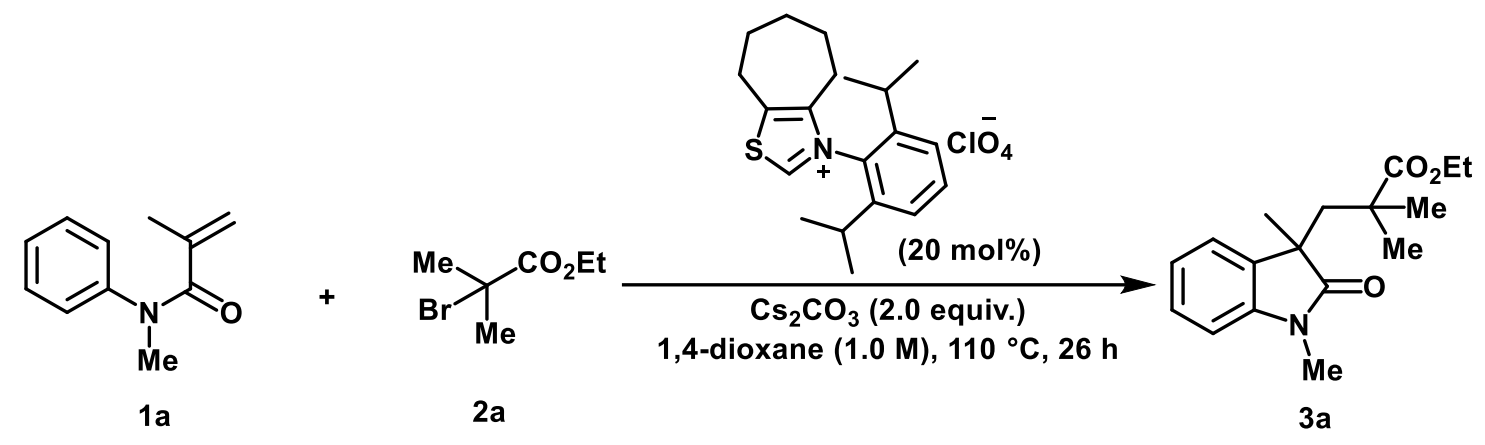

$\mathrm{N}$-arylacrylamide 1a $(6.0 \mathrm{mmol}, 1.05 \mathrm{~g})$, NHC A (1.2 mol, $496 \mathrm{mg})$ and $\mathrm{Cs}_{2} \mathrm{CO}_{3}(12.0$ $\mathrm{mmol}, 3.91 \mathrm{~g}$ ) were weighed into a $25 \mathrm{~mL}$ Schlenk tube. The reaction vessel was capped and subjected to three vacuum-purge/nitrogen-flush cycles. Then $2 \mathrm{a}(9.0 \mathrm{tmmol}, 1.3$ $\mathrm{mL}$ ) in 1, 4-dioxane $(6 \mathrm{~mL})$ was added through the side-arm by syringe. The reaction was stirred under nitrogen at $110{ }^{\circ} \mathrm{C}$ for $26 \mathrm{~h}$. Upon complete consumption of $\mathrm{N}$ arylacrylamide 1a compound, the reaction was cooled to room temperature. Volatile solvent and reagents were removed by rotary evaporation and the residue was purified by silica gel flash chromatography using petroleum ether/EtOAc (50:1 to 10:1) to afford the desired product $\mathbf{3 a}$ as a white solid, got: $1.30 \mathrm{~g}$, yield: $75 \%$. 


\section{Characterization of Products}<smiles>CCOC(=O)C(C)(C)CC1(C)C(=O)N(C)c2ccccc21</smiles>

ethyl 3-(1,3-dimethyl-2-oxoindolin-3-yl)-2,2-dimethylpropanoate (3a). ${ }^{3}$ Following the general procedure $A$, the product was purified by flash chromatography on silica gel (petroleum ether/EtOAc, 50:1 to 10:1), $67.3 \mathrm{mg}$, yield: $78 \%$, white solid. ${ }^{1} \mathrm{H}$ NMR $\left(\mathrm{CDCl}_{3}, 300 \mathrm{MHz}\right): \delta 7.25-7.20(\mathrm{~m}, 1 \mathrm{H}), 7.08(\mathrm{~d}, J=7.3 \mathrm{~Hz}, 1 \mathrm{H}), 6.97(\mathrm{t}, J=7.4 \mathrm{~Hz}, 1 \mathrm{H}), 6.82(\mathrm{~d}$, $J=7.7 \mathrm{~Hz}, 1 \mathrm{H}), 3.79-3.60(\mathrm{~m}, 2 \mathrm{H}), 3.19(\mathrm{~s}, 3 \mathrm{H}), 2.52(\mathrm{~d}, J=14.5 \mathrm{~Hz}, 1 \mathrm{H}), 2.20(\mathrm{~d}, J=14.5 \mathrm{~Hz}$, $1 \mathrm{H}), 1.28(\mathrm{~s}, 3 \mathrm{H}), 1.07-1.03(\mathrm{~m}, 6 \mathrm{H}), 0.80(\mathrm{~s}, 3 \mathrm{H}) ;{ }^{13} \mathrm{C} \mathrm{NMR}\left(\mathrm{CDCl}_{3}, 75 \mathrm{MHz}\right): \delta 180.6,177.1$, $143.2,131.9,127.8,124.3,121.8,108.0,60.2,47.2,47.0,41.5,29.3,27.7,26.2,22.2$, 13.7.<smiles>CCOC(=O)C(C)(C)CC1(C)C(=O)N(C)c2c([N+](=O)[O-])cccc21</smiles>

ethyl 2,2-dimethyl-3-(1,3,7-trimethyl-2-oxoindolin-3-yl)propanoate (3b). Following the general procedure $B$, the product was purified by flash chromatography on silica gel (petroleum ether/EtOAc, 50:1 to $10: 1), 55.5 \mathrm{mg}$, yield: $61 \%$, yellow oil. ${ }^{1} \mathrm{H}$ NMR $\left(\mathrm{CDCl}_{3}\right.$, $300 \mathrm{MHz}): \delta 6.95-6.82(\mathrm{~m}, 3 \mathrm{H}), 3.80-3.61(\mathrm{~m}, 2 \mathrm{H}), 3.47(\mathrm{~s}, 3 \mathrm{H}), 2.56(\mathrm{~s}, 3 \mathrm{H}), 2.47(\mathrm{~d}, J=14.4 \mathrm{~Hz}$, $1 \mathrm{H}), 2.20(\mathrm{~d}, J=14.4 \mathrm{~Hz}, 1 \mathrm{H}), 1.25(\mathrm{~s}, 3 \mathrm{H}), 1.09-1.02(\mathrm{~m}, 6 \mathrm{H}), 0.81(\mathrm{~s}, 3 \mathrm{H}) ;{ }^{13} \mathrm{C} \mathrm{NMR}\left(\mathrm{CDCl}_{3}, 75\right.$ MHz): $\delta 181.3,177.0,140.9,132.6,131.5,122.1,121.7,119.5,60.1,47.2,46.4,41.4,29.6$, 29.2, 28.0, 22.4, 19.1, 13.7. IR (ATR): 2973, 1721, 1698, 1443, 1374, 1233, 1158, 796, 749, 596 $\mathrm{cm}^{-1}$. HRMS (ESI) m/z: [M+H] ${ }^{+}$Calcd for $\mathrm{C}_{18} \mathrm{H}_{26} \mathrm{NO}_{3}$ 304.1907; Found 304.1911.<smiles>CCOC(=O)C(C)(C)CC1(C)C(=O)N(C)c2cc(C)ccc21</smiles>

ethyl 2,2-dimethyl-3-(1,3,6-trimethyl-2-oxoindolin-3-yl)propanoate (3c) and ethyl 2,2-dimethyl-3-(1,3,7-trimethyl-2-oxoindolin-3-yl)propanoate (3c'). ${ }^{4}$ Following the general procedure $A$, the product was purified by flash chromatography on silica gel 
(petroleum ether/EtOAc, 50:1 to 10:1). Isolated as a inseparable mixture, $\mathbf{3 c}: \mathbf{3} \mathbf{c}^{\mathbf{\prime}}=\mathbf{2 . 7}: \mathbf{1}$, $68.8 \mathrm{mg}$, total yield: $76 \%$, white solid. ${ }^{1} \mathrm{H} \mathrm{NMR}\left(\mathrm{CDCl}_{3}, 300 \mathrm{MHz}\right): \delta 7.14(\mathrm{t}, J=7.7 \mathrm{~Hz}, 0.7 \mathrm{H})$, $6.95(\mathrm{~d}, J=7.5 \mathrm{~Hz}, 0.6 \mathrm{H}), 6.77(\mathrm{t}, J=8.5 \mathrm{~Hz}, 1.3 \mathrm{H}), 6.67(\mathrm{~d}, J=9.1 \mathrm{~Hz}, 1.3 \mathrm{H}), 3.81-3.62(\mathrm{~m}, 2 \mathrm{H})$, 3.58-3.47 (m, 0.8H), $3.18(\mathrm{~s}, 4.1 \mathrm{H}), 2.59-2.47(\mathrm{~m}, 1.5 \mathrm{H}), 2.35(\mathrm{~d}, J=2.1 \mathrm{~Hz}, 4.1 \mathrm{H}), 2.18(\mathrm{~d}, J=$ $14.5 \mathrm{~Hz}, 1.5 \mathrm{H}), 1.37(\mathrm{~s}, 2.6 \mathrm{H}), 1.26(\mathrm{~s}, 3 \mathrm{H}), 1.07-1.00(\mathrm{~m}, 8.7 \mathrm{H}), 0.90-0.80(\mathrm{~m}, 6 \mathrm{H}) ;{ }^{13} \mathrm{C} \mathrm{NMR}$ ( $\left.\mathrm{CDCl}_{3}, 75 \mathrm{MHz}\right): \delta$ 180.9, 180.1, 177.1, 176.5, 143.5, 143.2, 137.8, 135.2, 129.4, 128.9, 127.7, 124.9, 124.1, 122.3, 108.9, 106.7, 60.2, 47.7, 47.0, 46.1, 41.6, 41.5, 29.6, 29.4, 28.5, 27.7, 26.2, $26.2,24.5,23.2,22.1,21.7,18.6,13.7,13.7$<smiles>CCOC(=O)C(C)(C)CC1(C)C(=O)N(C)c2ccc(C)cc21</smiles>

ethyl 2,2-dimethyl-3-(1,3,5-trimethyl-2-oxoindolin-3-yl)propanoate (3d). ${ }^{3}$ Following the general procedure $A$, the product was purified by flash chromatography on silica gel (petroleum ether/EtOAc, 50:1 to $10: 1$ ), $60.6 \mathrm{mg}$, yield: $67 \%$, white solid. ${ }^{1} \mathrm{H}$ NMR $\left(\mathrm{CDCl}_{3}, 300 \mathrm{MHz}\right): \delta 7.02(\mathrm{~d}, J=7.9 \mathrm{~Hz}, 1 \mathrm{H}), 6.89(\mathrm{~s}, 1 \mathrm{H}), 6.70(\mathrm{~d}, J=7.9 \mathrm{~Hz}, 1 \mathrm{H}), 3.78-3.61(\mathrm{~m}$, 2H), $3.17(\mathrm{~s}, 3 \mathrm{H}), 2.51(\mathrm{~d}, J=14.5 \mathrm{~Hz}, 1 \mathrm{H}), 2.30(\mathrm{~s}, 3 \mathrm{H}), 2.18(\mathrm{~d}, J=14.5 \mathrm{~Hz}, 1 \mathrm{H}), 1.26(\mathrm{~s}, 3 \mathrm{H})$, 1.09-1.04 (m, 6H), $0.79(\mathrm{~s}, 3 \mathrm{H}) ;{ }^{13} \mathrm{C}$ NMR ( $\left.\mathrm{CDCl}_{3}, 75 \mathrm{MHz}\right): \delta$ 180.6, 177.1, 140.8, 131.9, 131.1, $128.1,125.2,107.7,60.0,47.2,46.9,41.4,29.5,27.7,26.2,22.0,21.0,13.7$.<smiles>CCOC(=O)C(C)(C)CC1(C)C(=O)N(C)c2ccc(OC)cc21</smiles>

ethyl 3-(5-methoxy-1,3-dimethyl-2-oxoindolin-3-yl)-2,2-dimethylpropanoate (3e). ${ }^{3}$ Following the general procedure $A$, the product was purified by flash chromatography on silica gel (petroleum ether/EtOAc, 50:1 to $10: 1), 63.8 \mathrm{mg}$, yield: $67 \%$, yellow solid. ${ }^{1} \mathrm{H}$ NMR (CDCl, $300 \mathrm{MHz}): \delta$ 6.78-6.70 (m, 3H), 3.83-3.65 (m, 5H), $3.17(\mathrm{~s}, 3 \mathrm{H}), 2.50(\mathrm{~d}, J=14.5$

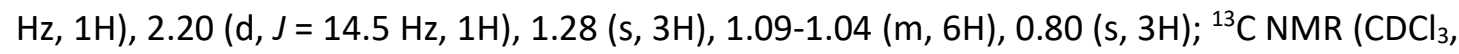
$75 \mathrm{MHz}$ ): $\delta 180.3,177.2,155.5,136.7,133.3,112.3,111.8,108.1,60.2,55.7,47.7,46.9,41.5$, 29.5, 27.7, 26.3, 22.1, 13.7. 
<smiles>CCOC(=O)C(C)(C)CC1(C)C(=O)N([N+](=O)[O-])c2ccc(F)cc21</smiles>

ethyl 3-(5-fluoro-1,3-dimethyl-2-oxoindolin-3-yl)-2,2-dimethylpropanoate (3f). ${ }^{5}$ Following the general procedure $A$, the product was purified by flash chromatography on silica gel (petroleum ether/EtOAc, $50: 1$ to $10: 1$ ), $57.8 \mathrm{mg}$, yield: $63 \%$, white solid. ${ }^{1} \mathrm{H}$ $\operatorname{NMR}\left(\mathrm{CDCl}_{3}, 300 \mathrm{MHz}\right): \delta 6.98-6.91(\mathrm{~m}, 1 \mathrm{H}), 6.84(\mathrm{dd}, J=8.1,2.5 \mathrm{~Hz}, 1 \mathrm{H}), 6.74$ (dd, $J=8.5,4.2$ $\mathrm{Hz}, 1 \mathrm{H}), 3.79(\mathrm{q}, J=7.1 \mathrm{~Hz}, 2 \mathrm{H}), 3.19(\mathrm{~s}, 3 \mathrm{H}), 2.51(\mathrm{~d}, J=14.5 \mathrm{~Hz}, 1 \mathrm{H}), 2.21(\mathrm{~d}, J=14.5 \mathrm{~Hz}, 1 \mathrm{H})$, $1.29(\mathrm{~s}, 3 \mathrm{H}), 1.10(\mathrm{t}, J=7.1 \mathrm{~Hz}, 3 \mathrm{H}), 1.05(\mathrm{~s}, 3 \mathrm{H}), 0.79(\mathrm{~s}, 3 \mathrm{H}) ;{ }^{19} \mathrm{~F} \mathrm{NMR}\left(\mathrm{CDCl}_{3}, 282 \mathrm{MHz}\right): \delta-$ 121.6 (s, 1F); $\left.{ }^{13} \mathrm{C} \mathrm{NMR} \mathrm{(CDCl} 3,75 \mathrm{MHz}\right): \delta 180.3,177.0,158.9$ (d, $J=238.5$ ), 139.1 (d, $J=1.7$ ), 133.6 (d, $J=7.7$ ), 114.0(d, $J=23.3$ ), 112.5 (d, $J=24.6$ ), 108.4 (d, $J=8.1), 60.3,47.7,46.8,41.5$, $29.5,27.6,26.4,22.0,13.8$.<smiles>CCOC(=O)C(C)(C)CC1(C)C(=O)N(C)c2ccc(C(F)(F)F)cc21</smiles>

ethyl 3-(1,3-dimethyl-2-oxo-5-(trifluoromethyl)indolin-3-yl)-2,2-dimethylpropanoate $(3 \mathrm{~g})$. Following the general procedure $A$, the product was purified by flash chromatography on silica gel (petroleum ether/EtOAc, 50:1 to 10:1), $79.1 \mathrm{mg}$, yield: 74\%, yellow solid. ${ }^{1} \mathrm{H}$ NMR $\left(\mathrm{CDCl}_{3}, 300 \mathrm{MHz}\right): \delta 7.52(\mathrm{~d}, J=8.0 \mathrm{~Hz}, 1 \mathrm{H}), 7.33(\mathrm{~s}, 1 \mathrm{H}), 6.90(\mathrm{~d}, J=$ $8.2 \mathrm{~Hz}, 1 \mathrm{H}), 3.80-3.65(\mathrm{~m}, 2 \mathrm{H}), 3.22(\mathrm{~s}, 3 \mathrm{H}), 2.57(\mathrm{~d}, J=14.6 \mathrm{~Hz}, 1 \mathrm{H}), 2.23(\mathrm{~d}, J=14.6 \mathrm{~Hz}, 1 \mathrm{H})$, $1.31(\mathrm{~s}, 3 \mathrm{H}), 1.08-1.04(\mathrm{~m}, 6 \mathrm{H}), 0.80(\mathrm{~s}, 3 \mathrm{H}) ;{ }^{19} \mathrm{~F} \mathrm{NMR}\left(\mathrm{CDCl}_{3}, 282 \mathrm{MHz}\right): \delta-61.2(\mathrm{~s}, 3 \mathrm{~F}) ;{ }^{13} \mathrm{C} \mathrm{NMR}$ $\left(\mathrm{CDCl}_{3}, 75 \mathrm{MHz}\right): \delta 180.5,176.8,146.2,132.5,125.8(\mathrm{q}, J=3.9), 124.2(\mathrm{q}, J=32.1), 122.6$, 121.3(q, J = 3.0), 107.9, 60.3, 47.3, 46.8, 41.5, 29.5, 27.7, 26.5, 22.2, 13.7. IR (ATR): 2925, 1714, 1622, 1458, 1375, 1324, 1161, 1080, 833, $752 \mathrm{~cm}^{-1}$. HRMS (ESI) m/z: $[\mathrm{M}+\mathrm{H}]^{+}$Calcd for $\mathrm{C}_{18} \mathrm{H}_{23} \mathrm{~F}_{3} \mathrm{NO}_{3} 358.1625$; Found 358.1631 .<smiles>CCOC(=O)C(C)(C)CC1(C)C(=O)N(C)c2ccc([N+](=O)[O-])cc21</smiles>

ethyl 3-(1,3-dimethyl-5-nitro-2-oxoindolin-3-yl)-2,2-dimethylpropanoate (3h). Following the general procedure $A$, the product was purified by flash chromatography on silica gel (petroleum ether/EtOAc, 50:1 to $10: 1$ ), $43.9 \mathrm{mg}$, yield: $44 \%$, yellow solid. ${ }^{1} \mathrm{H}$ 
$\operatorname{NMR}\left(\mathrm{CDCl}_{3}, 300 \mathrm{MHz}\right): \delta 8.24$ (dd, $\left.J=8.7,2.2 \mathrm{~Hz}, 1 \mathrm{H}\right), 7.97(\mathrm{~d}, J=2.0 \mathrm{~Hz}, 1 \mathrm{H}), 6.91$ (d, $J=8.6$ $\mathrm{Hz}, 1 \mathrm{H}), 3.82-3.66(\mathrm{~m}, 2 \mathrm{H}), 3.27(\mathrm{~s}, 3 \mathrm{H}), 2.58(\mathrm{~d}, J=14.6 \mathrm{~Hz}, 1 \mathrm{H}), 2.27(\mathrm{~d}, J=14.6 \mathrm{~Hz}, 1 \mathrm{H}), 1.35$ $(\mathrm{s}, 3 \mathrm{H}), 1.13(\mathrm{t}, J=7.1 \mathrm{~Hz}, 3 \mathrm{H}), 1.05(\mathrm{~s}, 3 \mathrm{H}), 0.80(\mathrm{~s}, 3 \mathrm{H}) ;{ }^{13} \mathrm{C} \mathrm{NMR}\left(\mathrm{CDCl}_{3}, 75 \mathrm{MHz}\right): \delta 180.6$, 176.5, 148.9, 142.9, 132.8, 125.4, 120.0, 107.7, 60.5, 47.2, 46.7, 41.4, 29.2, 27.5, 26.7, 22.5, 13.7. IR (ATR): 2980, 1715, 1615, 1518, 1331, 1294, 1135, 1046, 841, $752 \mathrm{~cm}^{-1}$. HRMS (ESI) m/z: $[\mathrm{M}+\mathrm{H}]^{+}$Calcd for $\mathrm{C}_{17} \mathrm{H}_{23} \mathrm{~N}_{2} \mathrm{O}_{5} 335.1601$; Found 335.1603.<smiles></smiles>

ethyl 3-(1-ethyl-3-methyl-2-oxoindolin-3-yl)-2,2-dimethylpropanoate (3i). ${ }^{3}$ Following the general procedure $A$, the product was purified by flash chromatography on silica gel (petroleum ether/EtOAc, 50:1 to $10: 1$ ), $60.0 \mathrm{mg}$, yield: $66 \%$, white solid. ${ }^{1} \mathrm{H}$ NMR $\left(\mathrm{CDCl}_{3}, 300 \mathrm{MHz}\right): \delta 7.24-7.19(\mathrm{~m}, 1 \mathrm{H}), 7.09(\mathrm{~d}, J=7.0,1 \mathrm{H}), 6.96(\mathrm{t}, J=7.3 \mathrm{~Hz}, 1 \mathrm{H}), 6.84(\mathrm{~d}, J=$ $7.8 \mathrm{~Hz}, 1 \mathrm{H}), 3.89-3.61(\mathrm{~m}, 4 \mathrm{H}), 2.53(\mathrm{~d}, J=14.5 \mathrm{~Hz}, 1 \mathrm{H}), 2.21(\mathrm{~d}, J=14.5 \mathrm{~Hz}, 1 \mathrm{H}), 1.27-1.23(\mathrm{~m}$, $6 \mathrm{H}), 1.07-1.03(\mathrm{~m}, 6 \mathrm{H}), 0.82(\mathrm{~s}, 3 \mathrm{H}) ;{ }^{13} \mathrm{C} \mathrm{NMR}\left(\mathrm{CDCl}_{3}, 75 \mathrm{MHz}\right): \delta 180.2,177.2,142.2,132.1$, $127.7,124.6,121.5,108.1,60.2,47.2,46.7,41.6,34.6,29.4,28.0,22.2,13.7,12.1$.<smiles></smiles>

ethyl 3-(1-cyclohexyl-3-methyl-2-oxoindolin-3-yl)-2,2-dimethylpropanoate (3j). Following the general procedure $A$, the product was purified by flash chromatography on silica gel (petroleum ether/EtOAc, $50: 1$ to $10: 1$ ), $49.9 \mathrm{mg}$, yield: $47 \%$, yellow oil. ${ }^{1} \mathrm{H}$ $\operatorname{NMR}\left(\mathrm{CDCl}_{3}, 300 \mathrm{MHz}\right): \delta 7.19(\mathrm{t}, J=7.7 \mathrm{~Hz}, 1 \mathrm{H}), 7.08(\mathrm{~d}, J=7.4 \mathrm{~Hz}, 1 \mathrm{H}), 7.03(\mathrm{~d}, J=7.9 \mathrm{~Hz}, 1 \mathrm{H})$, $6.94(\mathrm{t}, J=7.4 \mathrm{~Hz}, 1 \mathrm{H}), 4.22(\mathrm{t}, J=12.5 \mathrm{~Hz}, 1 \mathrm{H}), 3.81-3.60(\mathrm{~m}, 2 \mathrm{H}), 2.52(\mathrm{~d}, J=14.5 \mathrm{~Hz}, 1 \mathrm{H})$, 2.22-2.09 (m, 3H), 1.91-1.87 (m, 2H), 1.76-1.72 (m, 2H), 1.44-1.30 (m, 4H), $1.26(\mathrm{~s}, 3 \mathrm{H}), 1.08-$ $1.03(\mathrm{~m}, 6 \mathrm{H}), 0.83(\mathrm{~s}, 3 \mathrm{H}) ;{ }^{13} \mathrm{C} \mathrm{NMR}\left(\mathrm{CDCl}_{3}, 75 \mathrm{MHz}\right): \delta 180.4,177.3,142.2,132.4,127.4,124.6$, 121.1, 109.9, 60.2, 51.9, 46.9, 46.7, 41.6, 29.5, 28.9, 28.4, 26.0, 25.4, 22.3, 13.7. IR (ATR): 2926, 2856, 1709, 1609, 1466, 1382, 1191, 1132, 1027, 753, $741 \mathrm{~cm}^{-1}$. HRMS (ESI) m/z: $[\mathrm{M}+\mathrm{H}]^{+}$Calcd for $\mathrm{C}_{22} \mathrm{H}_{32} \mathrm{NO}_{3} 358.2377$; Found 358.2375. 
<smiles>CCOC(=O)C(C)(C)CC1(C)C(=O)N(Cc2ccccc2)c2ccccc21</smiles>

ethyl 3-(1-benzyl-3-methyl-2-oxoindolin-3-yl)-2,2-dimethylpropanoate (3k) ${ }^{4}$ Following the general procedure $B$, the product was purified by flash chromatography on silica gel (petroleum ether/EtOAc, $50: 1$ to $10: 1$ ), $46.9 \mathrm{mg}$, yield: $43 \%$, yellow oil. ${ }^{1} \mathrm{H}$ NMR $\left(\mathrm{CDCl}_{3}, 300 \mathrm{MHz}\right): \delta$ 7.31-7.24 (m, 5H), 7.15-7.10 (m, 2H), $6.96(\mathrm{t}, J=7.4 \mathrm{~Hz}, 1 \mathrm{H}), 6.75(\mathrm{~d}$, $J=7.7 \mathrm{~Hz}, 1 \mathrm{H}), 5.09(\mathrm{~d}, J=15.5 \mathrm{~Hz}, 1 \mathrm{H}), 4.73(\mathrm{~d}, J=15.5 \mathrm{~Hz}, 1 \mathrm{H}), 3.82-3.60(\mathrm{~m}, 2 \mathrm{H}), 2.57(\mathrm{~d}, J$ $=14.5 \mathrm{~Hz}, 1 \mathrm{H}), 2.28(\mathrm{~d}, J=14.5 \mathrm{~Hz}, 1 \mathrm{H}), 1.35(\mathrm{~s}, 3 \mathrm{H}), 1.07-1.02(\mathrm{~m}, 6 \mathrm{H}), 0.83(\mathrm{~s}, 3 \mathrm{H}) ;{ }^{13} \mathrm{C} \mathrm{NMR}$ ( $\left.\mathrm{CDCl}_{3}, 75 \mathrm{MHz}\right): \delta 180.6,177.1,142.3,135.9,132.0,128.6,127.6,127.5,127.5,124.4,121.8$, 109.0, 60.2, 47.2, 46.7, 43.9, 41.6, 29.3, 28.3, 22.6, 13.7 .<smiles>CCOC(=O)C1(C)CC(C)(C(=O)OCC)c2ccccc2N(c2ccccc2)C1=O</smiles>

ethyl 2,2-dimethyl-3-(3-methyl-2-oxo-1-phenylindolin-3-yl)propanoate (3I). . $^{3}$ Following the general procedure $A$, the product was purified by flash chromatography on silica gel (petroleum ether/EtOAc, $50: 1$ to $10: 1), 54.4 \mathrm{mg}$, yield: $52 \%$, yellow oil. ${ }^{1} \mathrm{H}$ $\operatorname{NMR}\left(\mathrm{CDCl}_{3}, 300 \mathrm{MHz}\right): \delta 7.52(\mathrm{t}, J=7.1 \mathrm{~Hz}, 2 \mathrm{H}), 7.43-7.37(\mathrm{~m}, 3 \mathrm{H}), 7.16(\mathrm{t}, J=7.2 \mathrm{~Hz}, 2 \mathrm{H}), 7.02$ $(\mathrm{t}, J=7.7 \mathrm{~Hz}, 1 \mathrm{H}), 6.82(\mathrm{~d}, J=7.6 \mathrm{~Hz}, 1 \mathrm{H}), 3.83-3.63(\mathrm{~m}, 2 \mathrm{H}), 2.62(\mathrm{~d}, J=14.5 \mathrm{~Hz}, 1 \mathrm{H}), 2.31(\mathrm{~d}, J$ $=14.5 \mathrm{~Hz}, 1 \mathrm{H}), 1.43(\mathrm{~s}, 3 \mathrm{H}), 1.11-1.05(\mathrm{~m}, 6 \mathrm{H}), 1.00(\mathrm{~s}, 3 \mathrm{H}) ;{ }^{13} \mathrm{C} \mathrm{NMR}\left(\mathrm{CDCl}_{3}, 75 \mathrm{MHz}\right): \delta 179.8$, 177.1, 143.1, 134.7, 131.7, 129.5, 127.8, 127.7, 126.3, 124.7, 122.2, 109.3, 60.3, 47.4, 47.2, $41.6,29.4,28.3,22.6,13.7$.<smiles>CCOC(=O)C(C)(C)CC1(C)C(=O)N(c2ccc(C)cc2)c2ccc(C)cc21</smiles>

ethyl 3-(3,5-dimethyl-2-oxo-1-(p-tolyl)indolin-3-yl)-2,2-dimethylpropanoate (3m). Following the general procedure $A$, the product was purified by flash chromatography on silica gel (petroleum ether/EtOAc, 50:1 to 10:1), $79.5 \mathrm{mg}$, yield: $70 \%$, yellow oil. ${ }^{1} \mathrm{H}$ 
$\operatorname{NMR}\left(\mathrm{CDCl}_{3}, 300 \mathrm{MHz}\right): \delta 7.34-7.26(\mathrm{~m}, 4 \mathrm{H}), 6.96(\mathrm{~d}, J=7.4 \mathrm{~Hz}, 2 \mathrm{H}), 6.69(\mathrm{~d}, J=8.4 \mathrm{~Hz}, 1 \mathrm{H})$, 3.82-3.65 (m, 2H), $2.60(\mathrm{~d}, J=14.5 \mathrm{~Hz}, 1 \mathrm{H}), 2.40(\mathrm{~s}, 3 \mathrm{H}), 2.32-2.26(\mathrm{~m}, 4 \mathrm{H}), 1.40(\mathrm{~s}, 3 \mathrm{H}), 1.11-$ 1.07 (m, 6H), 0.98 (s, 3H); $\left.{ }^{13} \mathrm{C} \mathrm{NMR} \mathrm{(CDCl} 3,75 \mathrm{MHz}\right): \delta 179.9,177.1,140.9,137.5,132.1,131.6$, 131.4, 130.0, 128.0, 125.9, 125.3, 109.0, 60.0, 47.3, 47.1, 41.5, 29.5, 28.2, 22.3, 21.1, 21.0, 13.7. IR (ATR): 2973, 1713, 1516, 1361, 1220, 1179, 811, 530, $514 \mathrm{~cm}^{-1}$. HRMS (ESI) m/z: $[\mathrm{M}+\mathrm{H}]^{+}$ Calcd for $\mathrm{C}_{24} \mathrm{H}_{30} \mathrm{NO}_{3} 380.2220$; Found 380.2223.<smiles>CN1C(=O)C(C)(CC(C)(CC2(C)CCCCOC2=O)C(=O)OCc2ccccc2)c2ccccc21</smiles>

benzyl 3-(1,3-dimethyl-2-oxoindolin-3-yl)-2,2-dimethylpropanoate (3n). Following the general procedure $A$, the product was purified by flash chromatography on silica gel (petroleum ether/EtOAc, 50:1 to 10:1), $74.3 \mathrm{mg}$, yield: $71 \%$, yellow oil. ${ }^{1} \mathrm{H} \mathrm{NMR}\left(\mathrm{CDCl}_{3}\right.$, $300 \mathrm{MHz}$ ): $\delta 7.35-7.28(\mathrm{~m}, 4 \mathrm{H}), 7.19(\mathrm{~d}, J=6.8 \mathrm{~Hz}, 2 \mathrm{H}), 7.11(\mathrm{~d}, J=7.3 \mathrm{~Hz}, 1 \mathrm{H}), 7.00(\mathrm{t}, J=7.4$ $\mathrm{Hz}, 1 \mathrm{H}), 6.81(\mathrm{~d}, J=7.7 \mathrm{~Hz}, 1 \mathrm{H}), 4.78(\mathrm{~d}, J=12.7 \mathrm{~Hz}, 1 \mathrm{H}), 4.63(\mathrm{~d}, J=12.7 \mathrm{~Hz}, 1 \mathrm{H}), 3.19(\mathrm{~s}, 3 \mathrm{H})$, $2.57(\mathrm{~d}, J=14.5 \mathrm{~Hz}, 1 \mathrm{H}), 2.27$ (d, J = 14.5 Hz, 1H), $1.30(\mathrm{~s}, 3 \mathrm{H}), 1.08(\mathrm{~s}, 3 \mathrm{H}), 0.88(\mathrm{~s}, 3 \mathrm{H}) ;{ }^{13} \mathrm{C}$ NMR ( $\left.\mathrm{CDCl}_{3}, 75 \mathrm{MHz}\right): \delta$ 180.4, 176.7, 143.1, 136.0, 131.8, 128.3, 127.9, 127.8, 127.5, 124.3, 121.8, 108.0, 65.9, 47.1, 47.0, 41.6, 29.2, 27.6, 26.2, 22.4. IR (ATR): 2969, 1710, 1613, 1472, $1453,1377,1349,1127,753,698,543 \mathrm{~cm}^{-1}$. HRMS (ESI) m/z: $[\mathrm{M}+\mathrm{H}]^{+}$Calcd for $\mathrm{C}_{22} \mathrm{H}_{26} \mathrm{NO}_{3}$ 352.1907; Found 352.1911.<smiles>CCOC(=O)C1(CC2(C)C(=O)N(C)c3ccccc32)CCC1</smiles>

ethyl 1-((1,3-dimethyl-2-oxoindolin-3-yl)methyl)cyclobutane-1-carboxylate (30). Following the general procedure $A$, the product was purified by flash chromatography on silica gel (petroleum ether/EtOAc, 50:1 to 10:1), $46.6 \mathrm{mg}$, yield: $52 \%$, yellow solid. ${ }^{1} \mathrm{H}$ $\operatorname{NMR}\left(\mathrm{CDCl}_{3}, 300 \mathrm{MHz}\right): \delta 7.22(\mathrm{t}, J=7.5 \mathrm{~Hz}, 1 \mathrm{H}), 7.07(\mathrm{~d}, J=7.1 \mathrm{~Hz}, 1 \mathrm{H}), 6.97(\mathrm{t}, J=7.4 \mathrm{~Hz}, 1 \mathrm{H})$, $6.78(\mathrm{~d}, J=7.7 \mathrm{~Hz}, 1 \mathrm{H}), 3.70-3.56(\mathrm{~m}, 2 \mathrm{H}), 3.16(\mathrm{~s}, 3 \mathrm{H}), 2.52(\mathrm{~d}, J=14.1 \mathrm{~Hz}, 1 \mathrm{H}), 2.45(\mathrm{~d}, J=14.1$ $\mathrm{Hz}, 1 \mathrm{H}), 2.13-2.02(\mathrm{~m}, 1 \mathrm{H}), 1.98-1.85(\mathrm{~m}, 3 \mathrm{H}), 1.70(\mathrm{q}, J=7.9 \mathrm{~Hz}, 2 \mathrm{H}), 1.31(\mathrm{~s}, 3 \mathrm{H}), 1.09(\mathrm{t}, J=$ $7.1 \mathrm{~Hz}, 3 \mathrm{H}) ;{ }^{13} \mathrm{C} \mathrm{NMR}\left(\mathrm{CDCl}_{3}, 75 \mathrm{MHz}\right): \delta 180.2,176.1,143.5,131.9,127.9,124.0,121.6,107.8$, 60.1, 47.1, 47.0, 46.0, 34.6, 28.2, 26.1, 25.6, 16.4, 13.7. IR (ATR): 2933, 1708, 1611, 1469, 1377, $1343,1213,1119,748,697,543 \mathrm{~cm}^{-1}$. HRMS (ESI) m/z: $[\mathrm{M}+\mathrm{H}]^{+}$Calcd for $\mathrm{C}_{18} \mathrm{H}_{24} \mathrm{NO}_{3} 302.1751$; Found 302.1751 . 
<smiles>COC(=O)C1(CC2(C)C(=O)N(C)c3ccccc32)CCCCC1</smiles>

methyl 1-((1,3-dimethyl-2-oxoindolin-3-yl)methyl)cyclohexane-1-carboxylate (3p). Following the general procedure $B$, the product was purified by flash chromatography on silica gel (petroleum ether/EtOAc, 50:1 to $10: 1$ ), $51.1 \mathrm{mg}$, yield: $54 \%$, white solid. ${ }^{1} \mathrm{H}$ $\operatorname{NMR}\left(\mathrm{CDCl}_{3}, 300 \mathrm{MHz}\right): \delta 7.23(\mathrm{t}, J=7.56 \mathrm{~Hz}, 1 \mathrm{H}), 7.07-6.97(\mathrm{~m}, 2 \mathrm{H}), 6.82(\mathrm{~d}, J=7.7 \mathrm{~Hz}, 1 \mathrm{H})$, $3.23(\mathrm{~s}, 3 \mathrm{H}), 3.20(\mathrm{~s}, 3 \mathrm{H}), 2.26(\mathrm{~s}, 2 \mathrm{H}), 1.79-1.68(\mathrm{~m}, 2 \mathrm{H}), 1.44-1.31(\mathrm{~m}, 4 \mathrm{H}), 1.26(\mathrm{~s}, 3 \mathrm{H}), 1.13-$ $0.98(\mathrm{~m}, 4 \mathrm{H}) ;{ }^{13} \mathrm{C} \mathrm{NMR}\left(\mathrm{CDCl}_{3}, 75 \mathrm{MHz}\right): \delta 180.1,175.9,143.1,132.4,127.7,123.5,121.9,108.0$, 51.2, 48.1, 46.7, 46.3, 37.7, 32.7, 28.2, 26.2, 25.4, 23.2, 23.0. IR (ATR): 2929, 1711, 1613, 1450, $1346,1217,1131,1023,752,700 \mathrm{~cm}^{-1}$. HRMS (ESI) m/z: [M+H] ${ }^{+}$Calcd for $\mathrm{C}_{19} \mathrm{H}_{26} \mathrm{NO}_{3} 316.1907$; Found 316.1914.<smiles>CC(C)C(C)(CC1(C)C(=O)N(C)c2ccccc21)C(=O)c1ccccc1</smiles>

3-(2,2-dimethyl-3-oxo-3-phenylpropyl)-1,3-dimethylindolin-2-one (3q). ${ }^{6}$ Following the general procedure $B$, the product was purified by flash chromatography on silica gel (petroleum ether/EtOAc, 50:1 to $10: 1), 33.6 \mathrm{mg}$, yield: $35 \%$, tawny oil. ${ }^{1} \mathrm{H} \mathrm{NMR}\left(\mathrm{CDCl}_{3}\right.$, $300 \mathrm{MHz}$ ): $\delta$ 7.36-7.31 (m, 1H), 7.26-7.21 (m, 3H), 7.04-6.92 (m, 4H), $6.84(\mathrm{~d}, J=7.8 \mathrm{~Hz}, 1 \mathrm{H})$, $3.20(\mathrm{~s}, 3 \mathrm{H}), 2.96(\mathrm{~d}, J=14.5 \mathrm{~Hz}, 1 \mathrm{H}), 2.37(\mathrm{~d}, J=14.5 \mathrm{~Hz}, 1 \mathrm{H}), 1.34(\mathrm{~s}, 3 \mathrm{H}), 1.16(\mathrm{~s}, 3 \mathrm{H}), 0.86(\mathrm{~s}$, $3 \mathrm{H}) ;{ }^{13} \mathrm{C} \mathrm{NMR}\left(\mathrm{CDCl}_{3}, 75 \mathrm{MHz}\right): \delta 209.5,181.0,143.2,139.1,132.3,130.1,122.8,127.7,127.0$, $125.8,122.3,108.0,47.5,47.3,46.0,29.7,28.0,26.3,23.9$.<smiles>[Y6]N1C(=O)C(C)(CC(C)C#N)c2ccccc21</smiles>

3-(1,3-dimethyl-2-oxoindolin-3-yl)-2-methylpropanenitrile $(3 r) .{ }^{7}$ Following the general procedure $A$, the product was purified by flash chromatography on silica gel (petroleum ether/EtOAc, 30:1 to 10:1), $42.3 \mathrm{mg}$, yield: 62\%, isolated as a mixture, $\mathrm{dr}=1: 1$, yellow oil. ${ }^{1} \mathrm{H}$ NMR $\left(\mathrm{CDCl}_{3}, 300 \mathrm{MHz}\right): \delta 7.36-7.25(\mathrm{~m}, 1.5 \mathrm{H}), 7.16-7.05(\mathrm{~m}, 1.5 \mathrm{H}), 6.87(\mathrm{~d}, J=$ $7.8 \mathrm{~Hz}, 1 \mathrm{H}), 3.22(\mathrm{~d}, J=1.86 \mathrm{~Hz}, 3 \mathrm{H}), 2.42-2.33(\mathrm{~m}, 1 \mathrm{H}), 2.29-2.11(\mathrm{~m}, 1 \mathrm{H}), 1.84(\mathrm{q}, J=9.7 \mathrm{~Hz}$, $1 \mathrm{H}), 1.40(\mathrm{~d}, J=6.6 \mathrm{~Hz}, 3 \mathrm{H}), 1.18-1.16(\mathrm{~m}, 3 \mathrm{H}) ;{ }^{13} \mathrm{C} \mathrm{NMR}\left(\mathrm{CDCl}_{3}, 75 \mathrm{MHz}\right): \delta 179.0,143.4,143.1$, 132.0, 131.3, 128.7, 128.5, 123.4, 123.0, 122.6, 122.4, 121.8, 121.6, 108.6, 108.4, 47.5, 47.0, 
<smiles>[Y6]N1C(=O)C(C)(CCC#N)c2ccccc21</smiles>

3-(1,3-dimethyl-2-oxoindolin-3-yl)propanenitrile (3s). ${ }^{7}$ Following the general procedure $A$, the product was purified by flash chromatography on silica gel (petroleum ether/EtOAc, 30:1 to 10:1), $14.1 \mathrm{mg}$, yield: $22 \%$, yellow oil. ${ }^{1} \mathrm{H}$ NMR $\left(\mathrm{CDCl}_{3}\right.$, $300 \mathrm{MHz}$ ): $\delta 7.31(\mathrm{t}, J=7.6 \mathrm{~Hz}, 1 \mathrm{H}), 7.19(\mathrm{~d}, J=7.1 \mathrm{~Hz}, 1 \mathrm{H}), 7.11(\mathrm{t}, J=7.4 \mathrm{~Hz}, 1 \mathrm{H}), 6.87(\mathrm{~d}, J=$ $7.7 \mathrm{~Hz}, 1 \mathrm{H}), 3.22(\mathrm{~s}, 3 \mathrm{H}), 2.36-2.27(\mathrm{~m}, 1 \mathrm{H}), 2.16-1.95(\mathrm{~m}, 3 \mathrm{H}), 1.39(\mathrm{~s}, 3 \mathrm{H}) ;{ }^{13} \mathrm{C} \mathrm{NMR}\left(\mathrm{CDCl}_{3}, 75\right.$ MHz): $\delta 178.8,143.1,131.6,128.6,123.0,122.5,118.7,108.4,47.3,33.4,26.2,23.4,12.8$.<smiles>[Y6]N1C(=O)C(C)(CC(Cl)(Cl)Cl)c2ccccc21</smiles>

1,3-dimethyl-3-(2,2,2-trichloroethyl)indolin-2-one (3t). ${ }^{8}$ Following the general procedure $A$, the product was purified by flash chromatography on silica gel (petroleum ether/EtOAc, 20:1 to 5:1), $44.4 \mathrm{mg}$, yield: $51 \%$, yellow solid. ${ }^{1} \mathrm{H}$ NMR $\left(\mathrm{CDCl}_{3}\right.$, $300 \mathrm{MHz}$ ): $\delta 7.35-7.27(\mathrm{~m}, 2 \mathrm{H}), 7.05(\mathrm{t}, J=7.5 \mathrm{~Hz}, 1 \mathrm{H}), 6.87(\mathrm{~d}, J=7.9 \mathrm{~Hz}, 1 \mathrm{H}), 3.68(\mathrm{~d}, J=15.3$ $\mathrm{Hz}, 1 \mathrm{H}), 3.33(\mathrm{~d}, J=15.3 \mathrm{~Hz}, 1 \mathrm{H}), 3.22(\mathrm{~s}, 3 \mathrm{H}), 1.38(\mathrm{~s}, 3 \mathrm{H}) ;{ }^{13} \mathrm{C} \mathrm{NMR}\left(\mathrm{CDCl}_{3}, 75 \mathrm{MHz}\right): \delta 178.5$, $143.2,129.4,128.4,125.6,121.9,108.3,96.1,59.8,47.9,26.8,26.5$.<smiles>CC1(CC(Br)(Br)Br)C(=O)N([N+](=O)[O-])c2ccccc21</smiles>

1,3-dimethyl-3-(2,2,2-tribromoethyl)indolin-2-one (3u). ${ }^{9}$ Following the general procedure $A$, the product was purified by flash chromatography on silica gel (petroleum ether/EtOAc, 30:1), $66.1 \mathrm{mg}$, yield: $52 \%$, white solid. ${ }^{1} \mathrm{H} \mathrm{NMR}\left(\mathrm{CDCl}_{3}, 300 \mathrm{MHz}\right)$ : $\delta 7.45(\mathrm{~d}, J=7.4 \mathrm{~Hz}, 1 \mathrm{H}), 7.31(\mathrm{t}, J=7.0 \mathrm{~Hz}, 1 \mathrm{H}), 7.05(\mathrm{t}, J=7.4 \mathrm{~Hz}, 1 \mathrm{H}), 6.87(\mathrm{~d}, J=7.8 \mathrm{~Hz}, 1 \mathrm{H})$, $4.12(\mathrm{~d}, J=15.1 \mathrm{~Hz}, 1 \mathrm{H}), 3.72(\mathrm{~d}, J=15.5 \mathrm{~Hz}, 1 \mathrm{H}), 3.23(\mathrm{~s}, 3 \mathrm{H}), 1.37(\mathrm{~s}, 3 \mathrm{H}) ;{ }^{13} \mathrm{C} \mathrm{NMR}\left(\mathrm{CDCl}_{3}, 75\right.$ $\mathrm{MHz}): \delta 178.3,143.5,128.6,128.6,126.5,121.8,108.3,63.6,50.0,32.8,27.1,26.6$. 
<smiles>CCOC(=O)C(C)(CC1(c2ccccc2)C(=O)N(C)c2ccccc21)C(=O)OCC</smiles>

ethyl 2,2-dimethyl-3-(1-methyl-2-oxo-3-phenylindolin-3-yl)propanoate (3v). ${ }^{10}$ Following the general procedure $A$, the product was purified by flash chromatography on silica gel (petroleum ether/EtOAc, $50: 1$ to $10: 1$ ), $50.4 \mathrm{mg}$, yield: $48 \%$, yellow oil. ${ }^{1} \mathrm{H}$ NMR $\left(\mathrm{CDCl}_{3}, 300 \mathrm{MHz}\right): \delta 7.37-7.18(\mathrm{~m}, 7 \mathrm{H}), 7.06(\mathrm{t}, J=7.6 \mathrm{~Hz}, 1 \mathrm{H}), 6.89(\mathrm{~d}, J=7.8 \mathrm{~Hz}, 1 \mathrm{H})$, 3.89-3.71 (m, 2H), $3.20(\mathrm{~s}, 3 \mathrm{H}), 2.95(\mathrm{~d}, J=14.3 \mathrm{~Hz}, 1 \mathrm{H}), 2.75(\mathrm{~d}, J=14.3 \mathrm{~Hz}, 1 \mathrm{H}), 1.11-1.07(\mathrm{~m}$, $6 \mathrm{H}), 0.90$ (s, 3H); ${ }^{13} \mathrm{C} \mathrm{NMR}\left(\mathrm{CDCl}_{3}, 75 \mathrm{MHz}\right): \delta 178.5,177.1,144.1,141.6,129.7,128.4,127.2$, $127.0,126.5,126.5,121.8,108.3,60.3,55.2,47.1,41.7,29.8,26.5,22.2,13.8$.<smiles>CCOC(=O)C1(C)CC2(C)C(=O)N(c3ccccc3)c3ncccc3C12C</smiles>

Ethyl 2,2-dimethyl-3-(3-methyl-2-oxo-1-phenyl-2,3-dihydro-1H-pyrrolo[2,3-b]pyridi n-3-yl)propanoate (3w). Following the general procedure $A$, the product was purified by flash chromatography on silica gel (petroleum ether/EtOAc, 50:1 to 10:1), $38.5 \mathrm{mg}$, yield: $37 \%$, yellow oil. $\left.{ }^{1} \mathrm{H} \mathrm{NMR} \mathrm{(} \mathrm{CDCl}_{3}, 300 \mathrm{MHz}\right): \delta 8.15(\mathrm{dd}, J=5.2,1.3 \mathrm{~Hz}, 1 \mathrm{H}), 7.53-7.51(\mathrm{~m}$, $4 \mathrm{H}), 7.45-7.35(\mathrm{~m}, 2 \mathrm{H}), 6.95(\mathrm{dd}, J=7.2,5.3 \mathrm{~Hz}, 1 \mathrm{H}), 3.85-3.61(\mathrm{~m}, 2 \mathrm{H}), 2.63(\mathrm{~d}, J=14.5 \mathrm{~Hz}$, $1 \mathrm{H}), 2.33(\mathrm{~d}, J=14.5 \mathrm{~Hz}, 1 \mathrm{H}), 1.44(\mathrm{~s}, 3 \mathrm{H}), 1.13(\mathrm{~s}, 3 \mathrm{H}), 1.05(\mathrm{t}, J=7.1 \mathrm{~Hz}, 3 \mathrm{H}), 1.00(\mathrm{~s}, 3 \mathrm{H}) ;{ }^{13} \mathrm{C}$ NMR (CDCl, $75 \mathrm{MHz}): \delta$ 179.3, 177.1, 156.4, 146.8, 133.3, 132.5, 129.1, 127.9, 126.3, 125.8, 118.1, 60.5, 46.9, 46.8, 41.6, 29.7, 27.8, 22.4, 13.7. IR (ATR): 2926, 1722, 1596, 1376, 1429, $1178,1145,779,699,639,499 \mathrm{~cm}^{-1}$. HRMS (ESI) m/z: [M+H]+ Calcd for $\mathrm{C}_{21} \mathrm{H}_{25} \mathrm{~N}_{2} \mathrm{O}_{3} 353.1860$; Found 353.1864 . 


\section{Diversity of the Products}

(a) Reduction of the product ${ }^{3}$

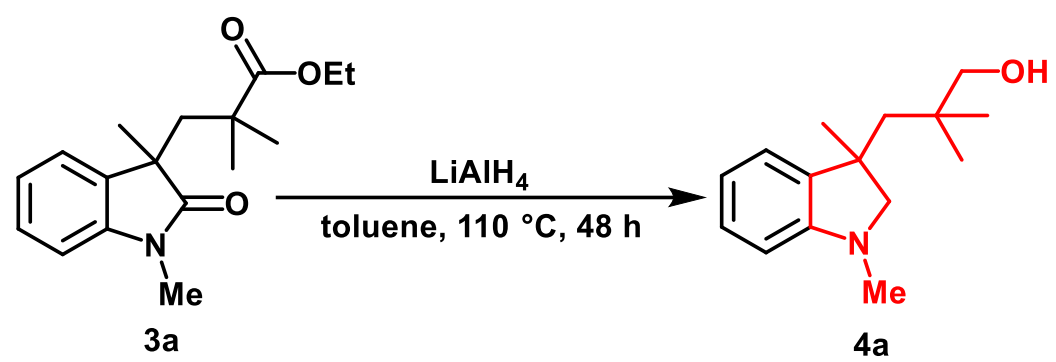

In a flame-dried schlenk tube, $3 a(0.3 \mathrm{mmol}, 86.8 \mathrm{mg})$ was dissolved in anhydrous toluene ( $3 \mathrm{~mL}$ ), $\mathrm{LiAlH}_{4}\left(3 \mathrm{mmol}, 113.9 \mathrm{mg}\right.$ ) was then added slowly at $0{ }^{\circ} \mathrm{C}$ under $\mathrm{N}_{2}$. The reaction mixture was stirred at $110^{\circ} \mathrm{C}$ for $48 \mathrm{~h}$. After cooling to room temperature, the reaction was quenched with a saturated solution of $\mathrm{NH}_{4} \mathrm{Cl}$. The reaction was then extracted with ether three times. The combined organic extracts were washed with brine, dried with $\mathrm{MgSO}_{4}$, filtrated and concentrated in vacuo. The product was purified by flash column chromatography (petroleum ether/EtOAc, 20:1) to yield $\mathbf{4 a}$ as a colorless oil.<smiles>CN1CC(C)(CC(C)(C)CO)c2ccccc21</smiles>

3-(1,3-dimethylindolin-3-yl)-2,2-dimethylpropan-1-ol (4a). $49.6 \mathrm{mg}$, yield: 71\%, colorless oil. ${ }^{1} \mathrm{H}$ NMR $\left(\mathrm{CDCl}_{3}, 300 \mathrm{MHz}\right): \delta 7.11(\mathrm{t}, J=7.6 \mathrm{~Hz}, 1 \mathrm{H}), 7.03(\mathrm{~d}, J=7.2 \mathrm{~Hz}, 1 \mathrm{H}), 6.72$ (t, $J=7.3 \mathrm{~Hz}, 1 \mathrm{H}$ ), 6.50 (d, J= $7.8 \mathrm{~Hz}, 1 \mathrm{H}$ ), $3.37(\mathrm{~d}, J=8.9 \mathrm{~Hz}, 1 \mathrm{H}), 3.26(\mathrm{~s}, 2 \mathrm{H}), 3.10(\mathrm{~d}, J=8.9$ $\mathrm{Hz}, 1 \mathrm{H}), 2.77(\mathrm{~s}, 3 \mathrm{H}), 1.91(\mathrm{~s}, 1 \mathrm{H}), 1.84-1.68(\mathrm{~m}, 2 \mathrm{H}), 1.37(\mathrm{~s}, 3 \mathrm{H}), 0.94(\mathrm{~s}, 6 \mathrm{H}) ;{ }^{13} \mathrm{C} \mathrm{NMR}\left(\mathrm{CDCl}_{3}\right.$, $75 \mathrm{MHz}$ ): $\delta$ 151.6, 139.5, 127.6, 122.0, 117.9, 107.4, 72.6, 68.9, 48.4, 43.9, 36.8, 35.9, 28.4, 25.9, 25.4. IR (ATR): 3398, 2954, 2889, 1605, 1492, 1456, 1297, 1272, 1121, 1041, 1022, 988, $740 \mathrm{~cm}^{-1}$. HRMS (ESI) m/z: [M+H] Calcd for $\mathrm{C}_{15} \mathrm{H}_{24} \mathrm{NO}$ 234.1852; Found 234.1860. 


\section{Mechanism Study}

(1) Control experiments

(a) Without NHC catalyst

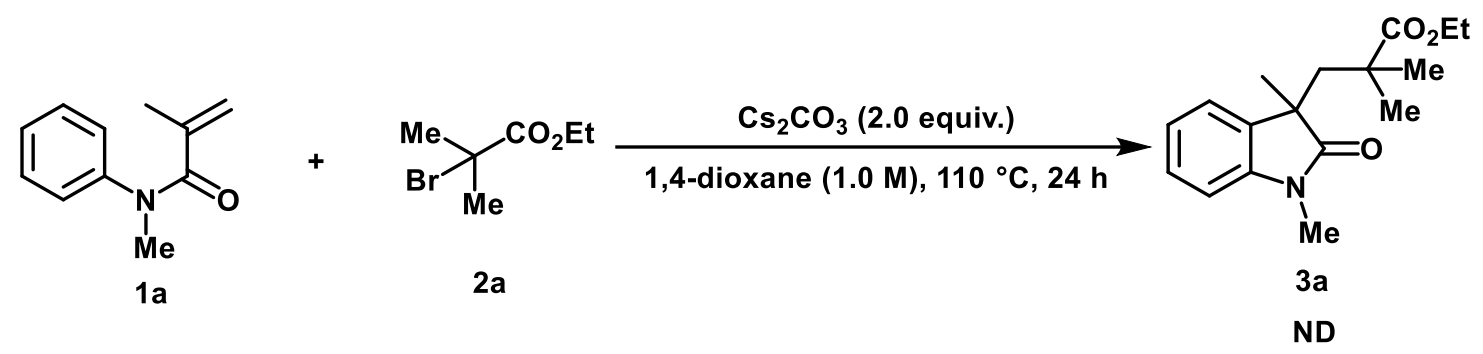

Procedures:

$\mathrm{N}$-arylacrylamide 1a (0.25 mmol, $43.8 \mathrm{mg}, 1.0$ equiv.), $\mathrm{Cs}_{2} \mathrm{CO}_{3}(0.5 \mathrm{mmol}, 162.5 \mathrm{mg}, 2.0$ equiv.) were weighed into a Schlenk tube. The reaction vessel was capped and subjected to three vacuum-purge/nitrogen-flush cycles. Then $2 \mathrm{a}(0.375 \mathrm{mmol}, 56 \mu \mathrm{L}$, 1.5 equiv.) in 1, 4-dioxane $(0.25 \mathrm{~mL})$ was added through the side-arm by syringe. The reaction was stirred under argon at $110{ }^{\circ} \mathrm{C}$ for $24 \mathrm{~h}$. After reaction, the mixture was extracted by EtOAc, then the solvent was evaporated under reduced pressure and it was submitted to ${ }^{1} \mathrm{H}$ NMR. No product was detected by NMR.

\section{(b) Without base}

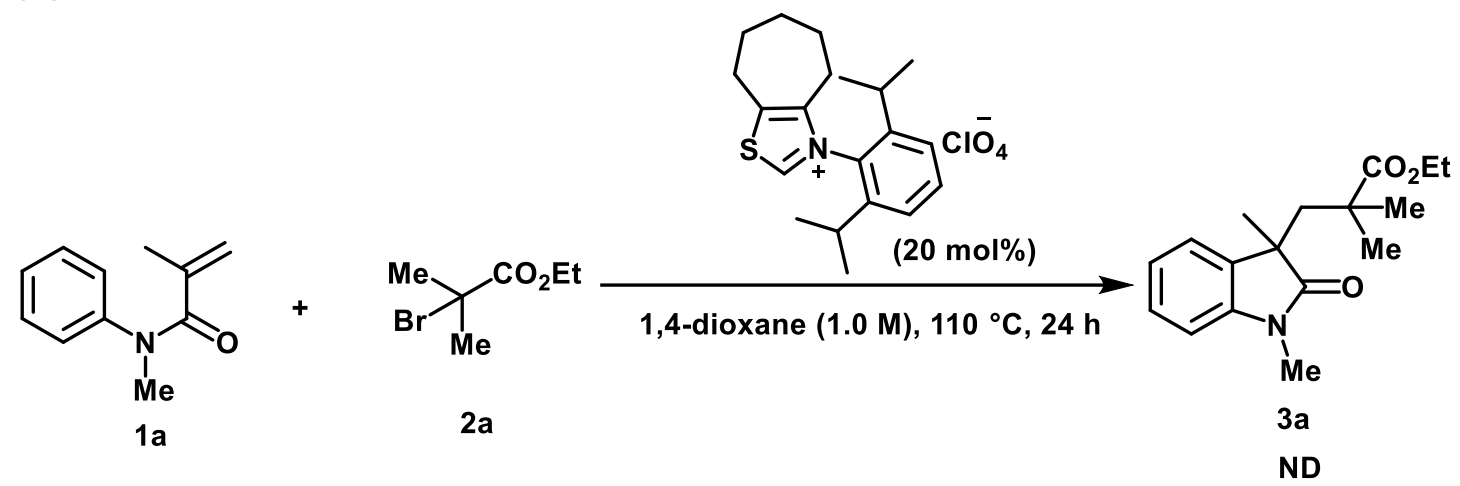

Procedures:

$\mathrm{N}$-arylacrylamide 1 a $(0.25 \mathrm{mmol}, 43.8 \mathrm{mg}, 1.0$ equiv.), NHC A (20.75 mg, $20 \mathrm{~mol} \%)$ were weighed into a Schlenk tube. The reaction vessel was capped and subjected to three vacuum-purge/nitrogen-flush cycles. Then $2 \mathrm{a}(0.375 \mathrm{mmol}, 56 \mu \mathrm{L}, 1.5$ equiv.) in 1, 4-dioxane $(0.25 \mathrm{~mL})$ was added through the side-arm by syringe. The reaction was stirred under argon at $110{ }^{\circ} \mathrm{C}$ for $24 \mathrm{~h}$. After reaction, the mixture was extracted by EtOAc, then the solvent was evaporated under reduced pressure and it was submitted to ${ }^{1} \mathrm{H}$ NMR. No product was detected by NMR. 
(c) Without NHC catalyst and base

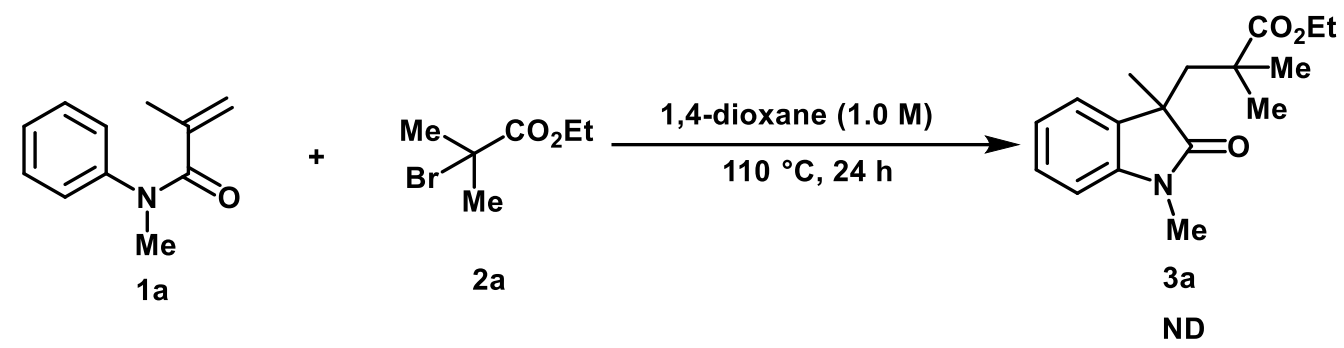

Procedures:

$\mathrm{N}$-arylacrylamide 1a ( $0.25 \mathrm{mmol}, 43.8 \mathrm{mg}, 1.0$ equiv.) was weighed into a Schlenk tube. The reaction vessel was capped and subjected to three vacuum-purge/nitrogen-flush cycles. Then $2 \mathrm{a}(0.375 \mathrm{mmol}, 56 \mu \mathrm{L}, 1.5$ equiv.) in 1, 4-dioxane $(0.25 \mathrm{~mL})$ was added through the side-arm by syringe. The reaction was stirred under argon at $110^{\circ} \mathrm{C}$ for 24 h. After reaction, the mixture was extracted by EtOAc, then the solvent was evaporated under reduced pressure and it was submitted to ${ }^{1} \mathrm{H}$ NMR. No product was detected by NMR.

(2) Radial capture experiments

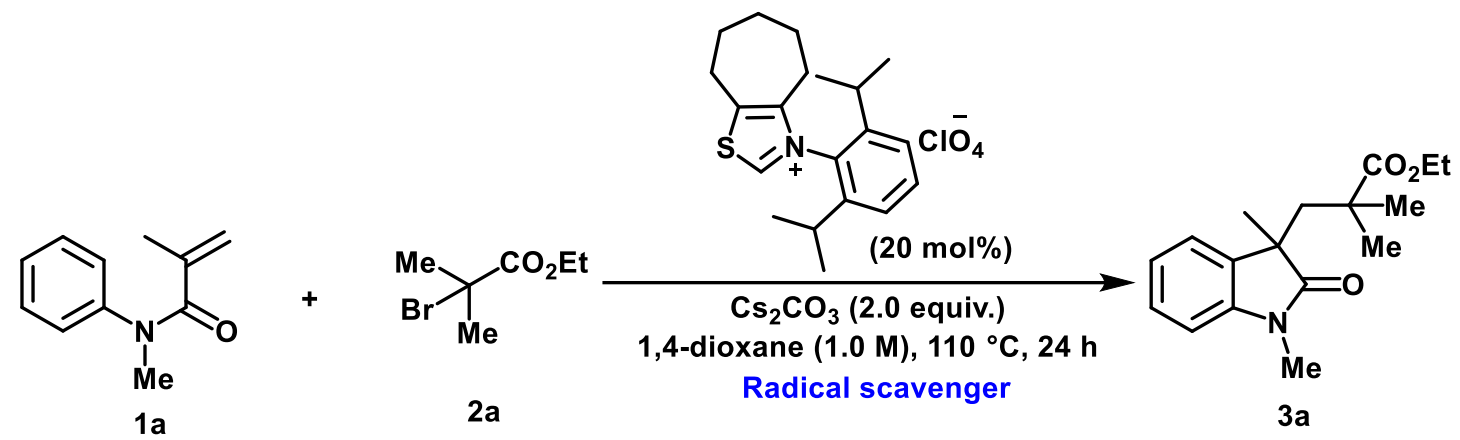

Procedures:

(a) TEMPO

$\mathrm{N}$-arylacrylamide 1a (0.3 mmol, $52.53 \mathrm{mg}, 1.0$ equiv.), NHC A (24.79 mg, $20 \mathrm{~mol} \%)$, TEMPO (70.32 mg, 1.5 equiv.) and $\mathrm{Cs}_{2} \mathrm{CO}_{3}$ ( $0.6 \mathrm{mmol}, 195.5 \mathrm{mg}, 2.0$ equiv.) were weighed into a Schlenk tube. The reaction vessel was capped and subjected to three vacuum-purge/nitrogen-flush cycles. Then 2 a $(0.45 \mathrm{mmol}, 66.04 \mu \mathrm{L}, 1.5$ equiv.) in 1 , 4-dioxane $(0.3 \mathrm{~mL})$ was added through the side-arm by syringe. The reaction was stirred under argon at $110^{\circ} \mathrm{C}$ for $24 \mathrm{~h}$. After reaction, the mixture was extracted by EtOAc, then the solvent was evaporated under reduced pressure and it was submitted to ${ }^{1} \mathrm{H}$ NMR. No product was detected by NMR. 


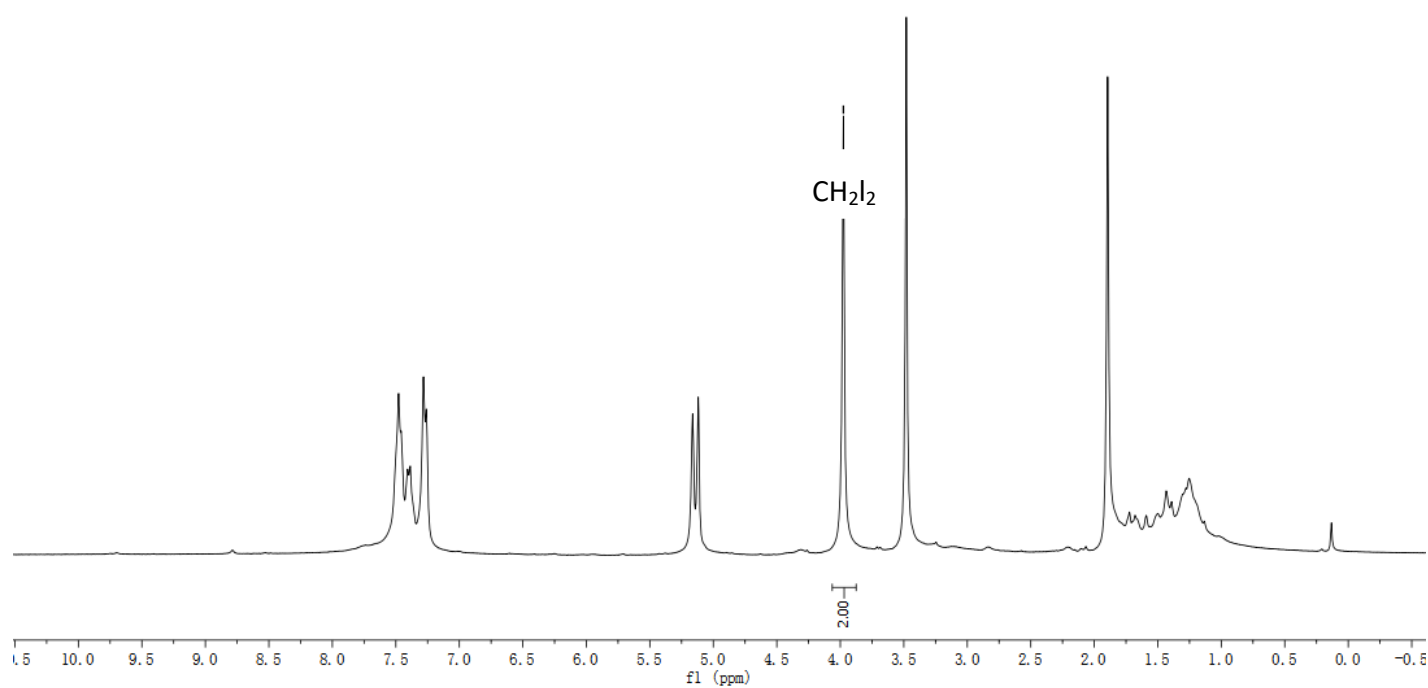

(b) BHT

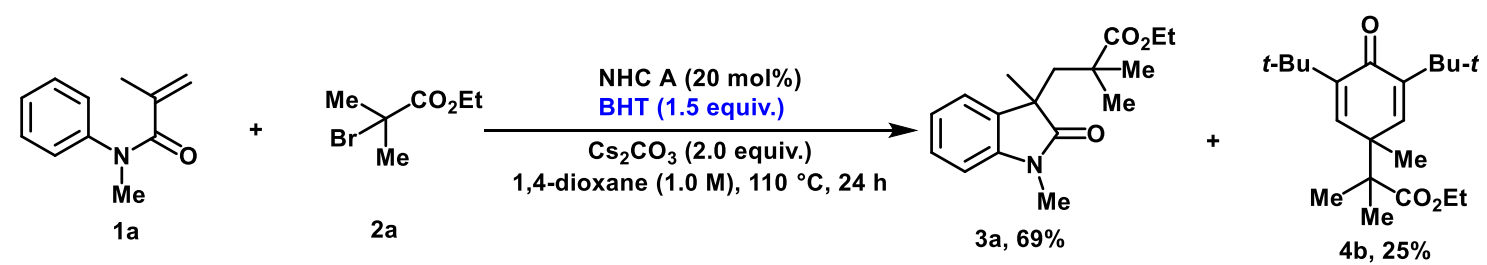

$\mathrm{N}$-arylacrylamide $1 \mathrm{a}(0.3 \mathrm{mmol}, 52.53 \mathrm{mg}, 1.0$ equiv.), NHC A (24.79 mg, $20 \mathrm{~mol} \%)$, $\mathrm{BHT}$ (99.16 mg, 1.5 equiv.) and $\mathrm{Cs}_{2} \mathrm{CO}_{3}(0.6 \mathrm{mmol}, 195.5 \mathrm{mg}, 2.0$ equiv.) were weighed into a Schlenk tube. The reaction vessel was capped and subjected to three vacuum-purge/nitrogen-flush cycles. Then $2 \mathrm{a}(0.45 \mathrm{mmol}, 66.04 \mu \mathrm{L}, 1.5$ equiv.) in 1 , 4-dioxane $(0.3 \mathrm{~mL})$ was added through the side-arm by syringe. The reaction was stirred under argon at $110^{\circ} \mathrm{C}$ for $24 \mathrm{~h}$. After reaction, the mixture was extracted by EtOAc, then the solvent was evaporated under reduced pressure and it was submitted to ${ }^{1} \mathrm{H}$ NMR. The product 3a was got in $69 \%$ NMR yield. 


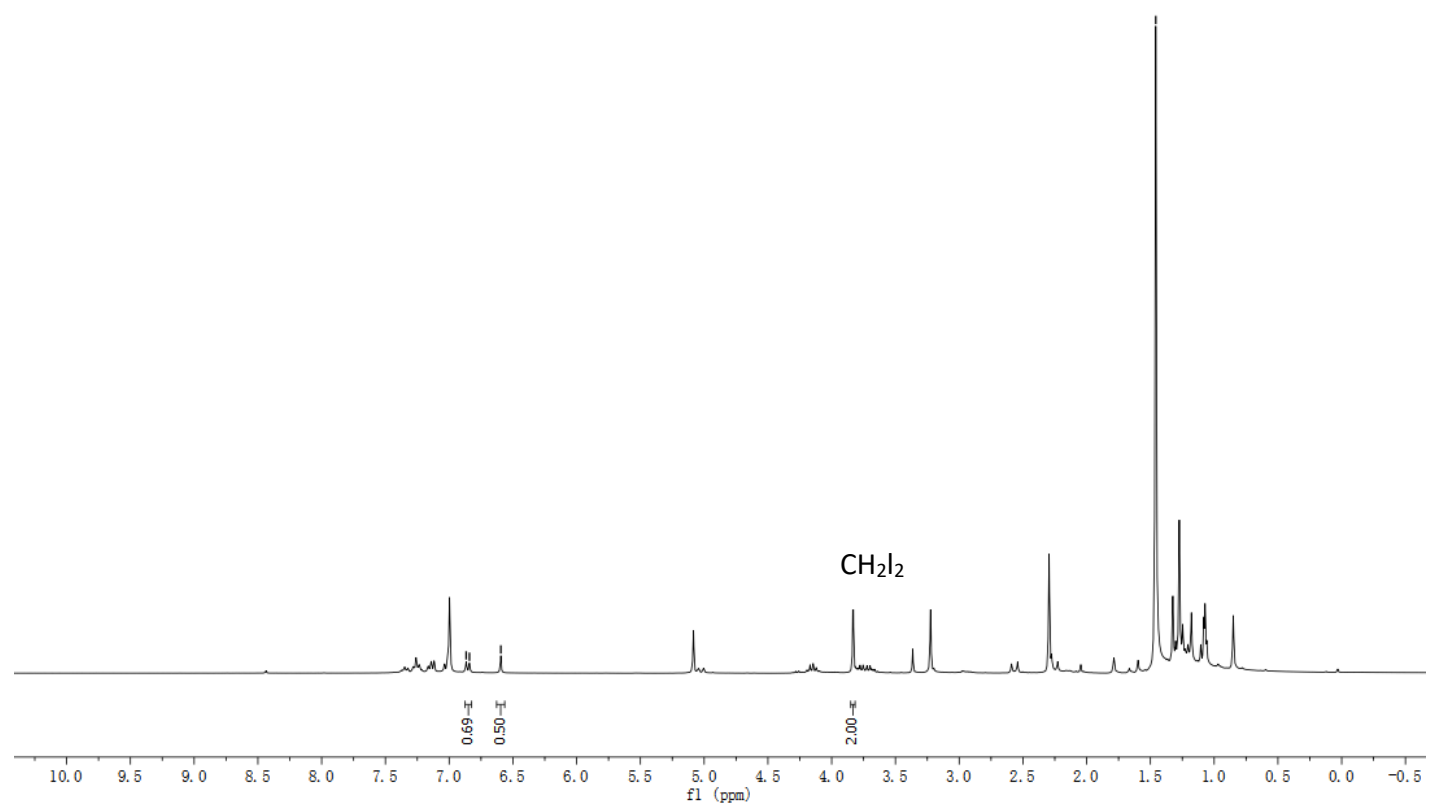<smiles>CCOC(C)(C)C1(C(C)(C)C)C=C(C(C)(C)C)C(=O)C(C(C)(C)C)=C1</smiles>

Ethyl 2-(2,6-di-tert-butyl-1-methyl-4-oxocyclohexa-2,5-dien-1-yl)-2-methylpropano ate (4b). ${ }^{10}{ }^{1} \mathrm{H}$ NMR yield: $25 \%$, isolated yield: $17 \%, 17.2 \mathrm{mg}$, The product was purified by flash chromatography on silica gel (pure petroleum ether), yellow oil. ${ }^{1} \mathrm{H} \mathrm{NMR}\left(\mathrm{CDCl}_{3}\right.$, $300 \mathrm{MHz}): \delta 6.55(\mathrm{~s}, 2 \mathrm{H}), 4.13(\mathrm{q}, J=7.1 \mathrm{~Hz}, 2 \mathrm{H}), 1.30-1.20(\mathrm{~m}, 24 \mathrm{H}), 1.13(\mathrm{~s}, 6 \mathrm{H}) ;{ }^{13} \mathrm{C} \mathrm{NMR}$ $\left(\mathrm{CDCl}_{3}, 75 \mathrm{MHz}\right): \delta 186.1,175.4,147.0,143.6,60.7,49.0,42.9,34.9,29.4,21.9,21.6,14.2$.

\section{(c) Under $\mathrm{O}_{2}$}

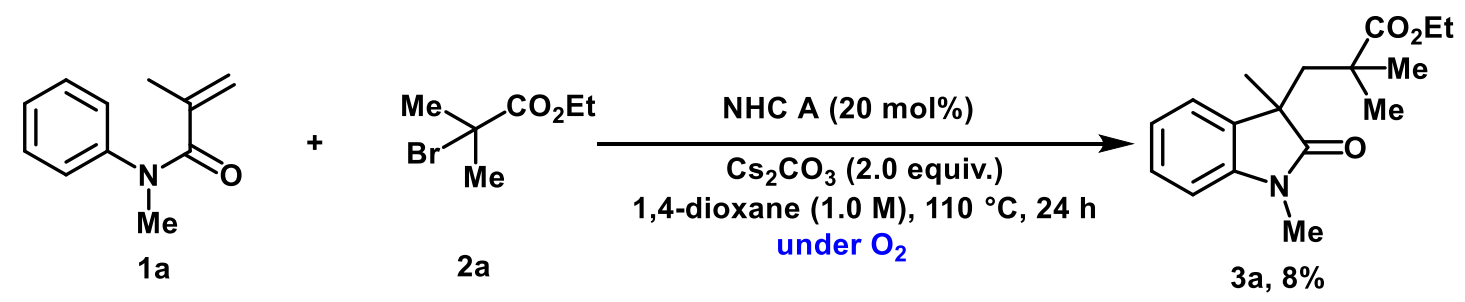

$\mathrm{N}$-arylacrylamide 1a (0.3 mmol, $52.53 \mathrm{mg}, 1.0$ equiv.), NHC A (24.79 mg, $20 \mathrm{~mol} \%$ ) and $\mathrm{Cs}_{2} \mathrm{CO}_{3}(0.6 \mathrm{mmol}, 195.5 \mathrm{mg}, 2.0$ equiv.) were weighed into a Schlenk tube. The reaction vessel was capped and subjected to $\mathrm{O}_{2}$ (via $\mathrm{O}_{2}$ balloon). Then $2 \mathrm{a}(0.45 \mathrm{mmol}$, $66.04 \mu \mathrm{L}, 1.5$ equiv.) in 1, 4-dioxane $(0.3 \mathrm{~mL})$ was added through the side-arm by 
syringe. The reaction was stirred under $\mathrm{O}_{2}$ at $110{ }^{\circ} \mathrm{C}$ for $24 \mathrm{~h}$. After reaction, the mixture was extracted by EtOAc, then the solvent was evaporated under reduced pressure and it was submitted to ${ }^{1} \mathrm{H}$ NMR. The NMR yield of product $3 \mathbf{3}$ was determined as $8 \%$.

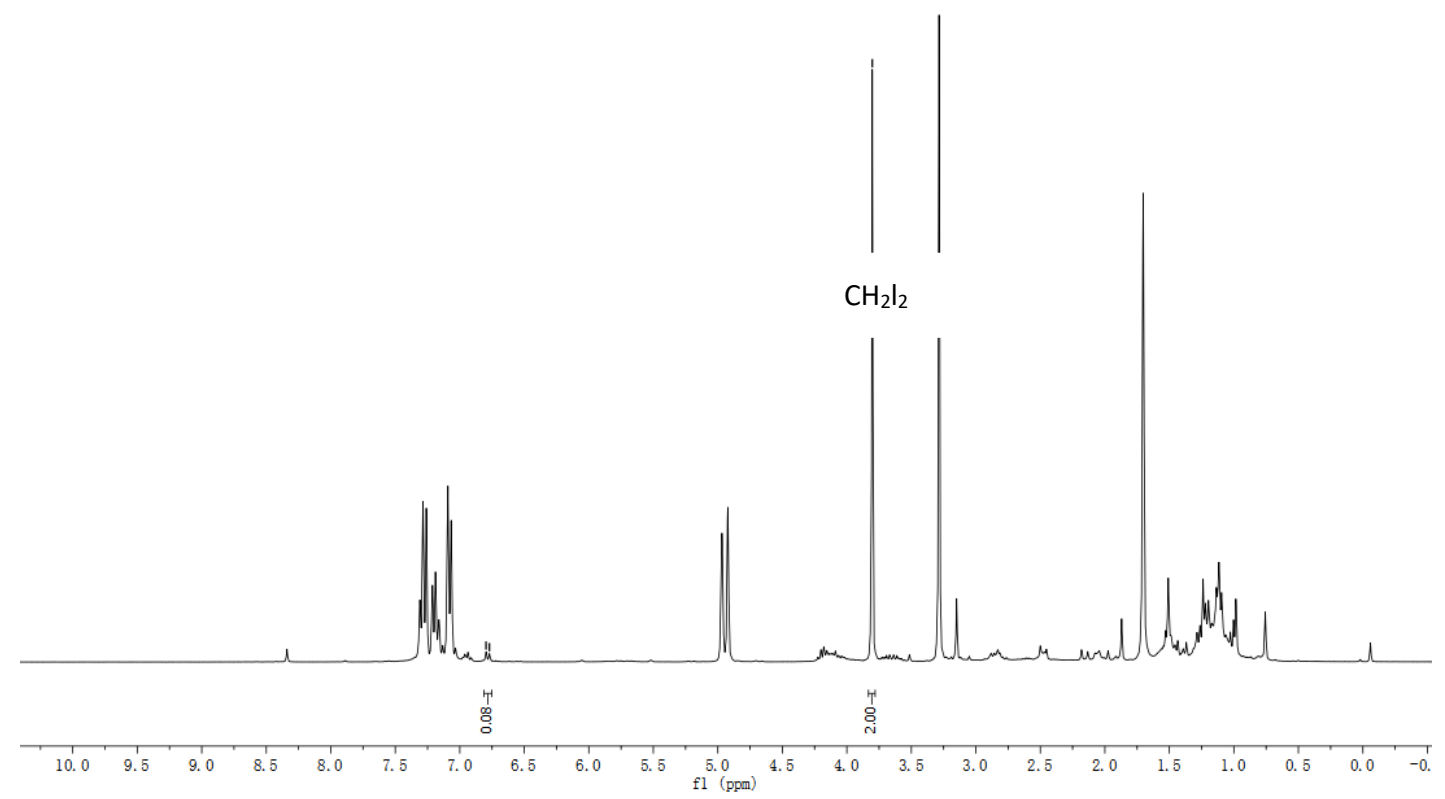

(3) Influences of the amounts of base

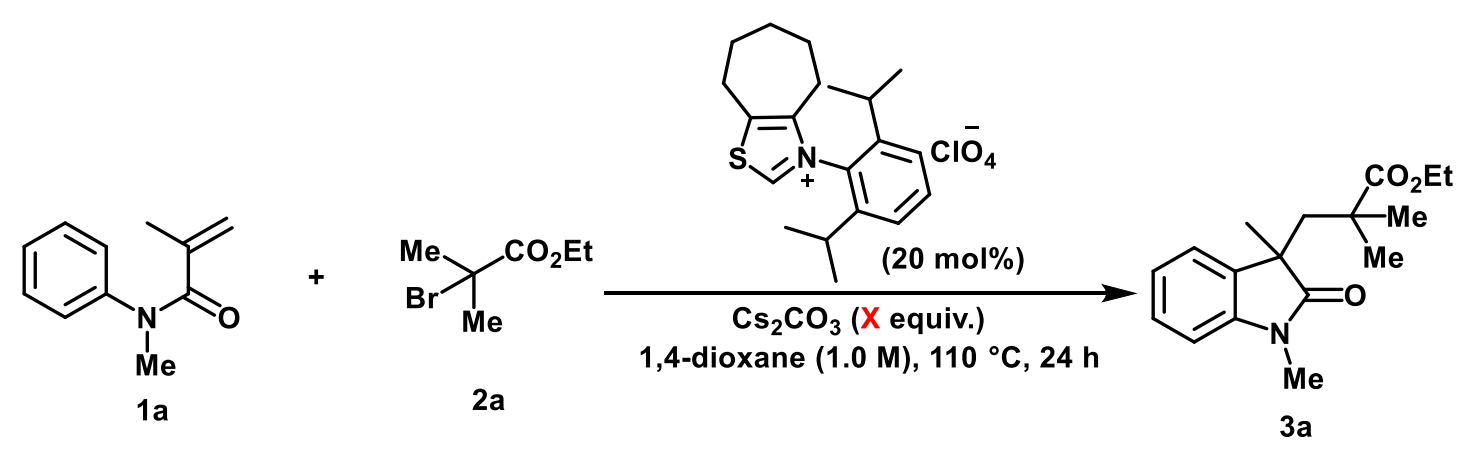




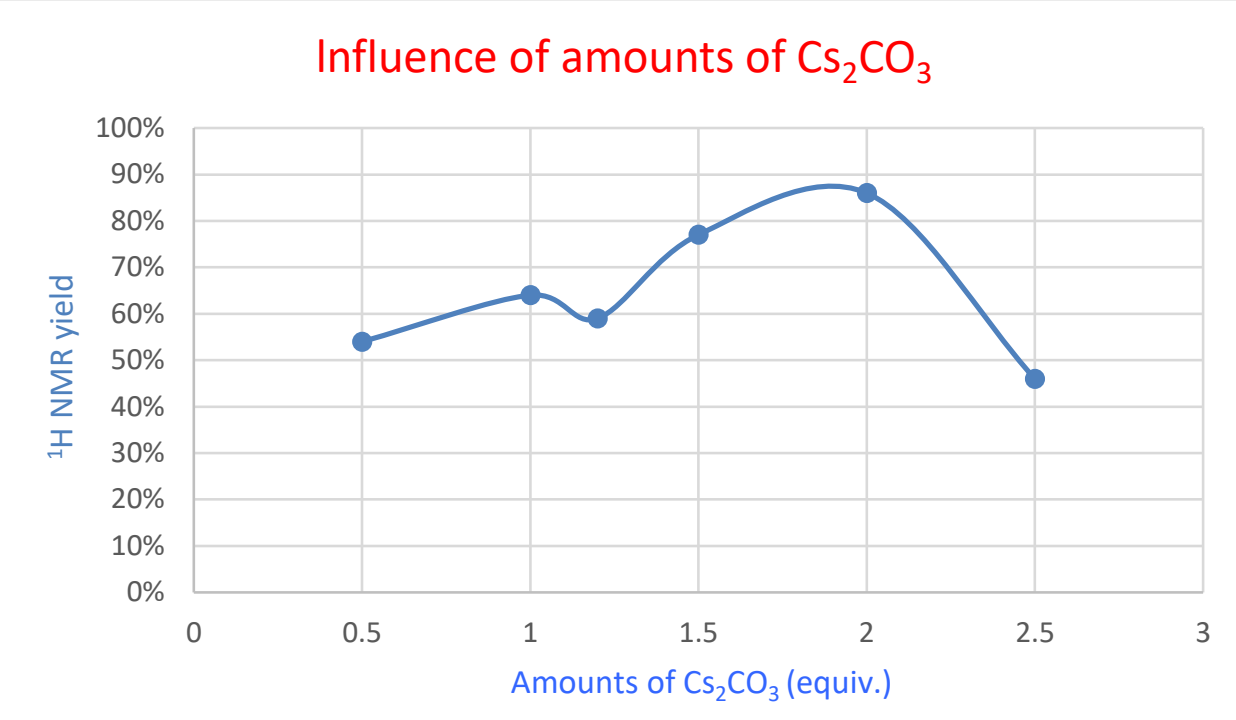

Procedure: following the General Procedure for NHC-Catalyzed Radical Cyclization.

(4) Influences of the amounts of NHC
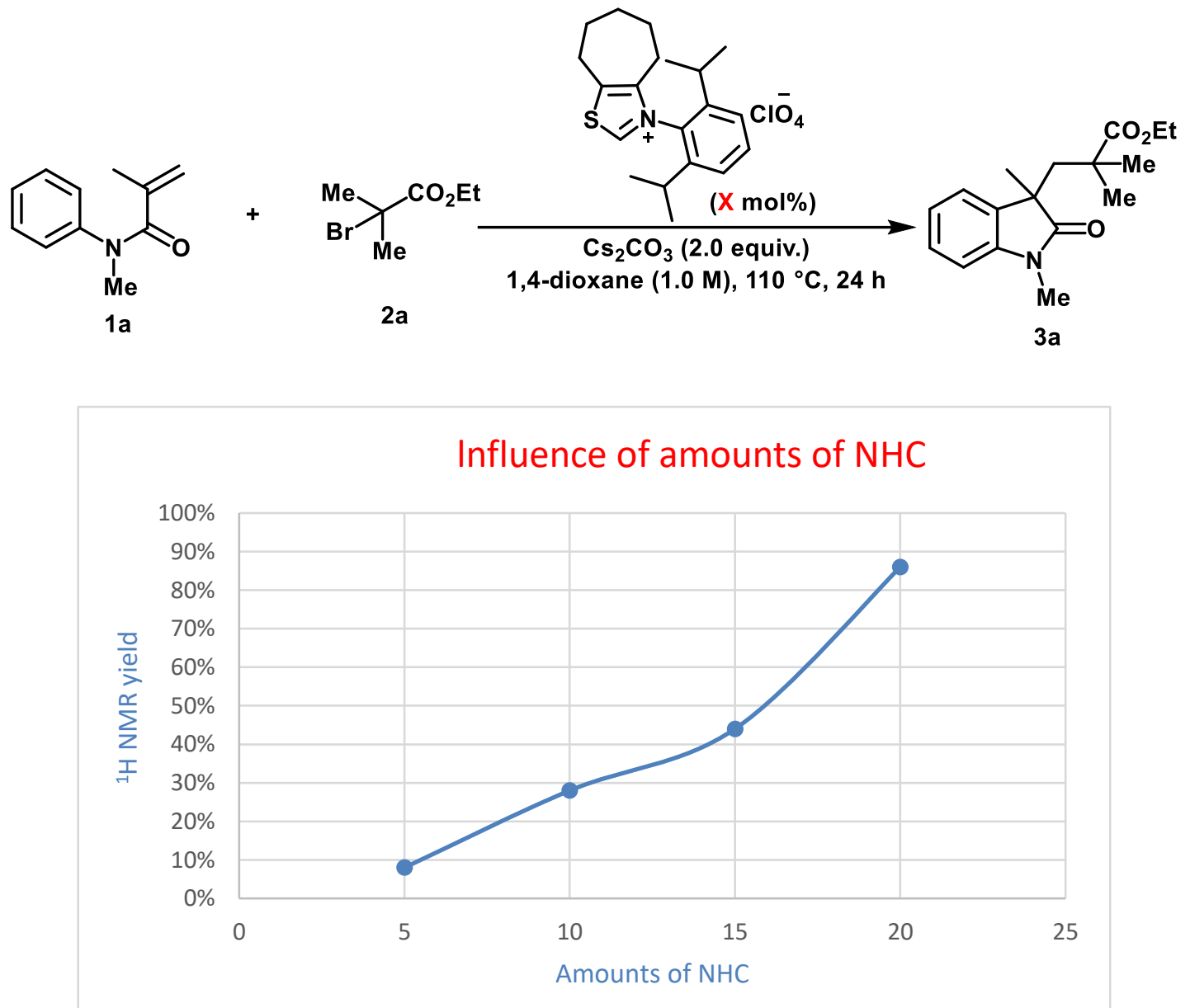

Procedure: Following the General Procedure for NHC-Catalyzed Radical Cyclization. 
(5) Selectivity of the cyclization reaction

(a) With different aromatic rings<smiles>C=C(C)C(=O)N(c1ccccc1)c1ccccn1</smiles>

$1 w$

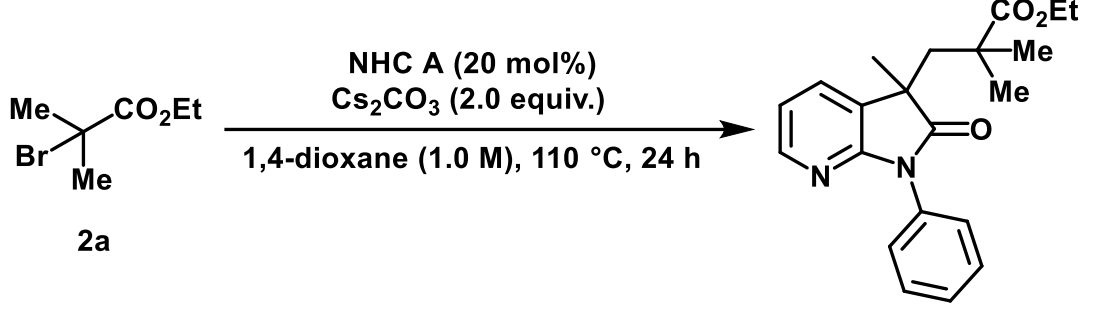

$3 w, 37 \%$

Procedure: Following the General Procedure for NHC-Catalyzed Radical Cyclization.

(b) With different substituted functional groups<smiles>C=C(C)C(=O)N(c1ccc(C(=C)C)cc1)c1ccc([N+](=O)[O-])cc1</smiles>

$1 \mathrm{x}$

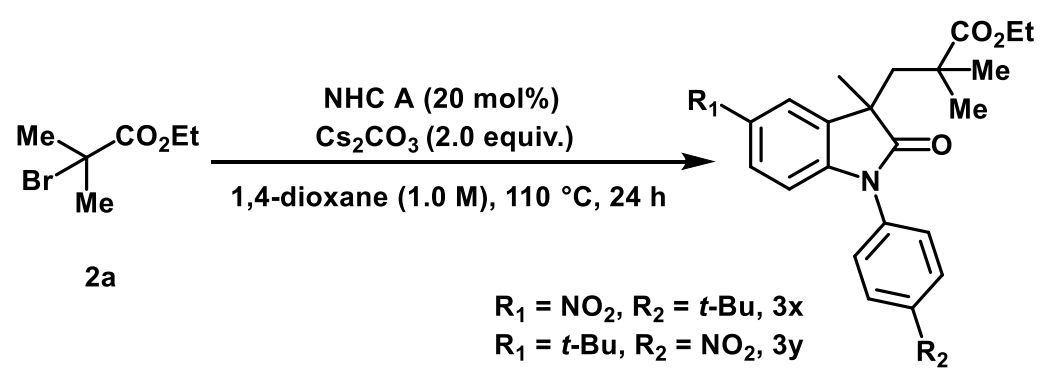

Yield $17 \%$,

$3 x: 3 y=1: 1$

sjj-50/1

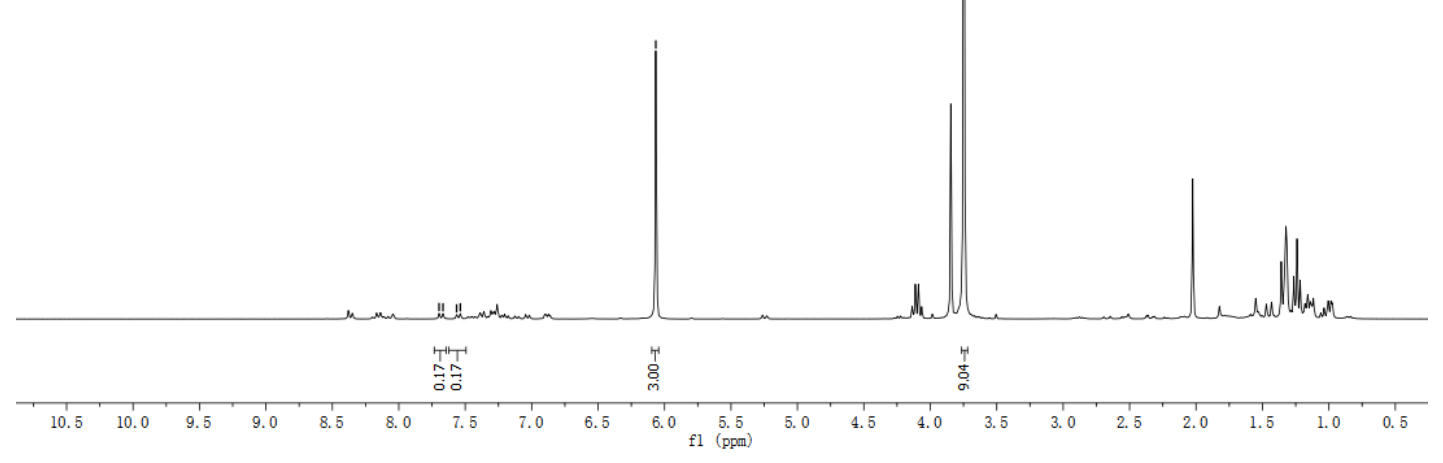

Procedure: Following the General Procedure for NHC-Catalyzed Radical Cyclization. 


\section{References}

(1) Kilaru, P.; Acharya, S. P.; Zhao, P. Chem. Commun. 2018, 54, 924-927.

(2) a) Piel, I., Pawelczyk, M. D., Hirano, K., Fröhlich, R., Glorius, F., Eur. J. Org. Chem. 2011, 28, 5475-5484; b) Wei, S.; Wei, X. -G.; Su, X.; You, J.; Ren, Y. Chem. Eur. J. 2011, 17, 5965-5971.

(3) Yoshinaga, K.; Tsubaki, N.; Murata, Y.; Noda, Y.; Nishikata, T. ACS Omega. 2018, 3, 9020-9026.

(4) Fan, J. -H.; Yang, J.; Song, R. -J.; Li, J. -H. Org. Lett. 2015, 17, 836-839.

(5) Liu, D.; Zhuang, S.; Chen, X.; Yu, L.; Yu, Y.; Hu, L.; Tan, Z. Tetrahedron Lett. 2018, 59, 612-616.

(6) Fan, J. -H.; Wei, W. -T.; Zhou, M. -B.; Song, R. -J.; Li, J. -H. Angew. Chem. Int. Ed. 2014, 53, 6650-6654.

(7) Li, J.; Wang, Z.; Wu, N.; Gao, G.; You, J. Chem. Commun. 2014, 50, 15049-15051.

(8) Li, D.; Shen, X. Tetrahedron Lett. 2020, 61, 152316.

(9) Chen, F.; Wang, Y.; Zhao, S.; Jiang, W.; Huo, C. Org. Biomol. Chem. 2017, 15, 77107714.

(10) Yamane, Y.; Yoshinaga, K.; Sumimoto, M.; Nishikata, T. ACS. Catal. 2019, 9, 17571762. 
NMR Spectra Images of Products

${ }^{1} \mathrm{H}$ NMR of compound $3 \mathrm{a}$ (300 MHz in $\left.\mathrm{CDCl}_{3}\right)$

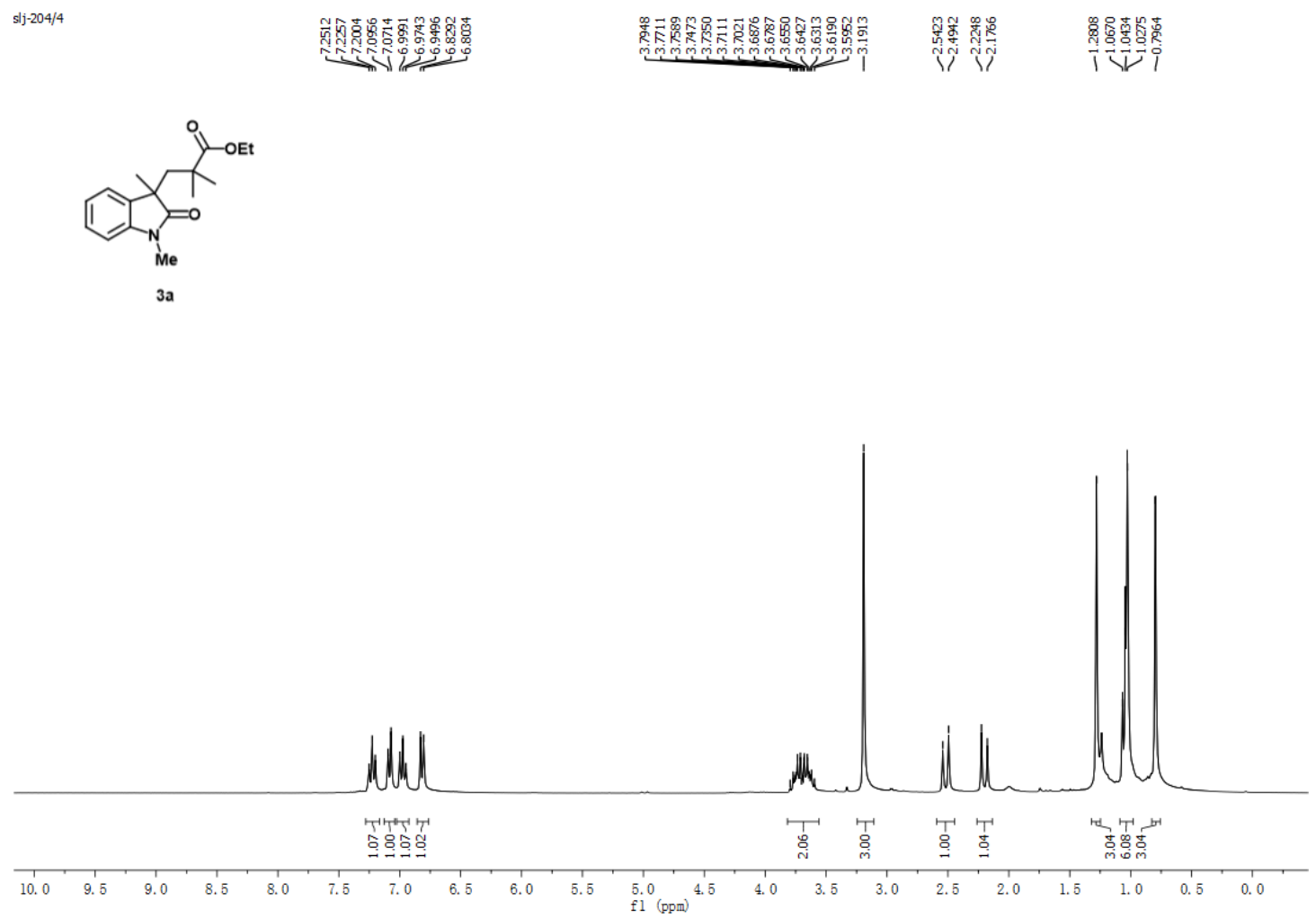

${ }^{13} \mathrm{C}$ NMR of compound $\mathbf{3 a}\left(75 \mathrm{MHz}\right.$ in $\left.\mathrm{CDCl}_{3}\right)$

$s \mid j-204-C / 4$

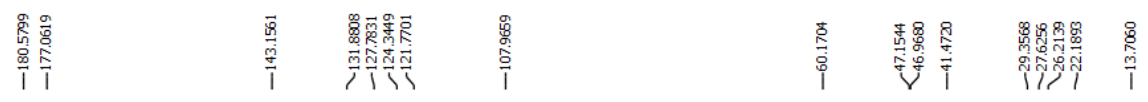

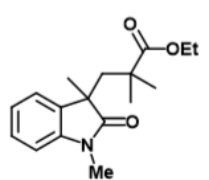

3a

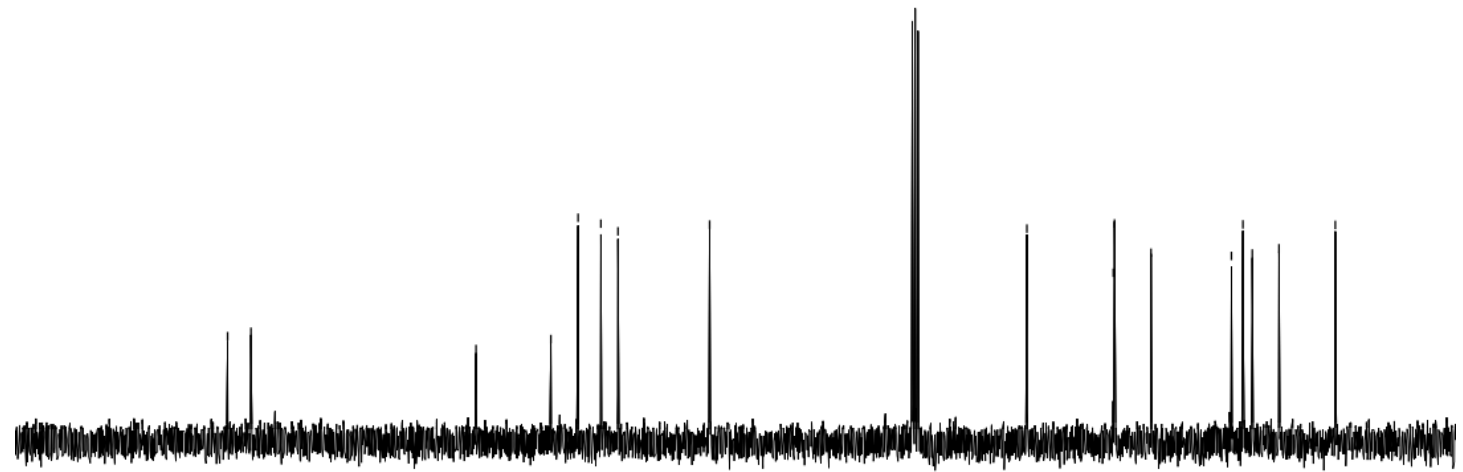

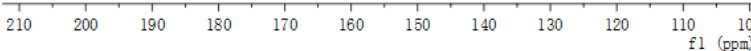


${ }^{1} \mathbf{H}$ NMR of compound $\mathbf{3 b}\left(300 \mathrm{MHz}\right.$ in $\left.\mathrm{CDCl}_{3}\right)$

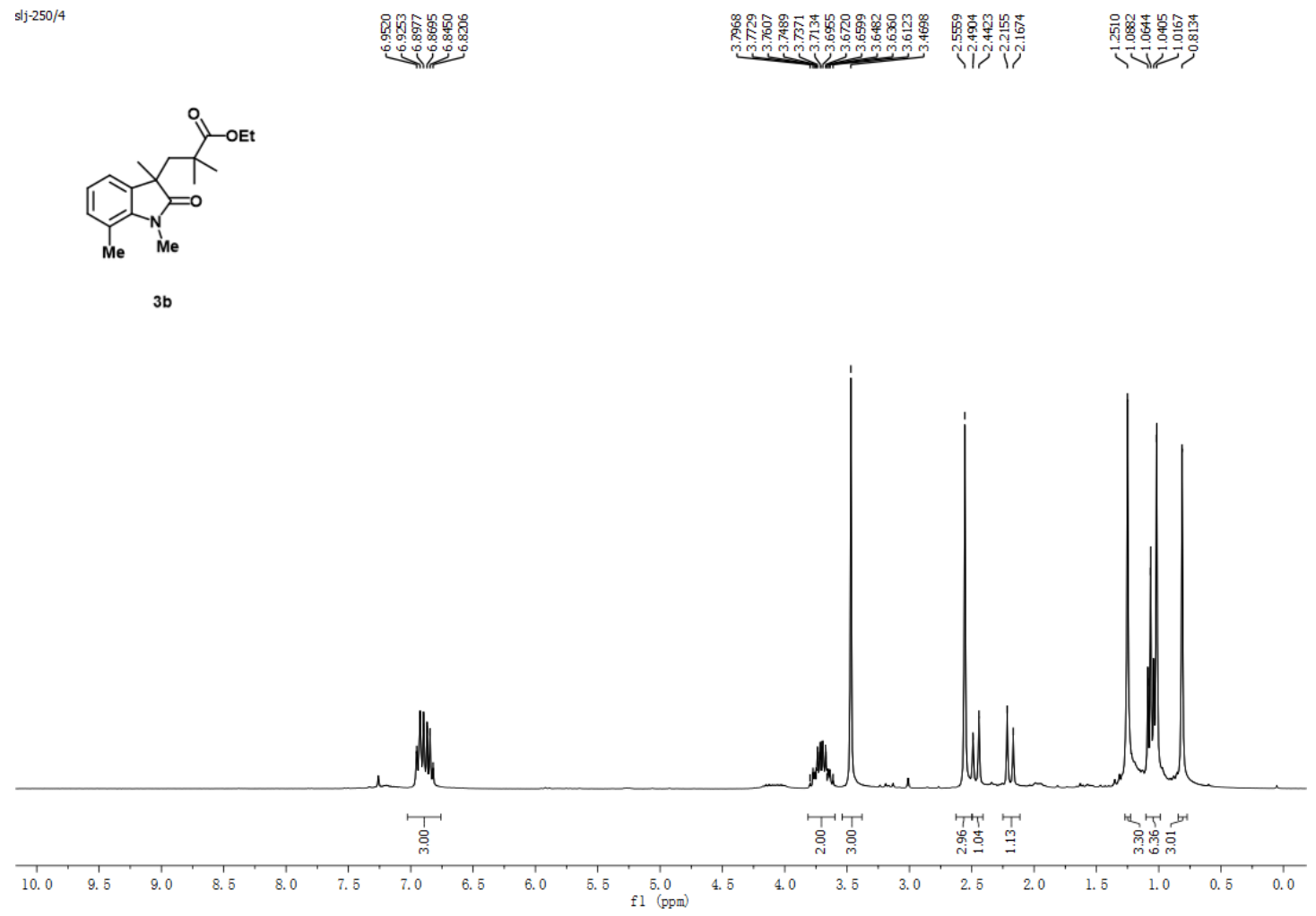

${ }^{13} \mathrm{C} \mathrm{NMR}$ of compound $\mathbf{3 b}\left(75 \mathrm{MHz}\right.$ in $\left.\mathrm{CDCl}_{3}\right)$
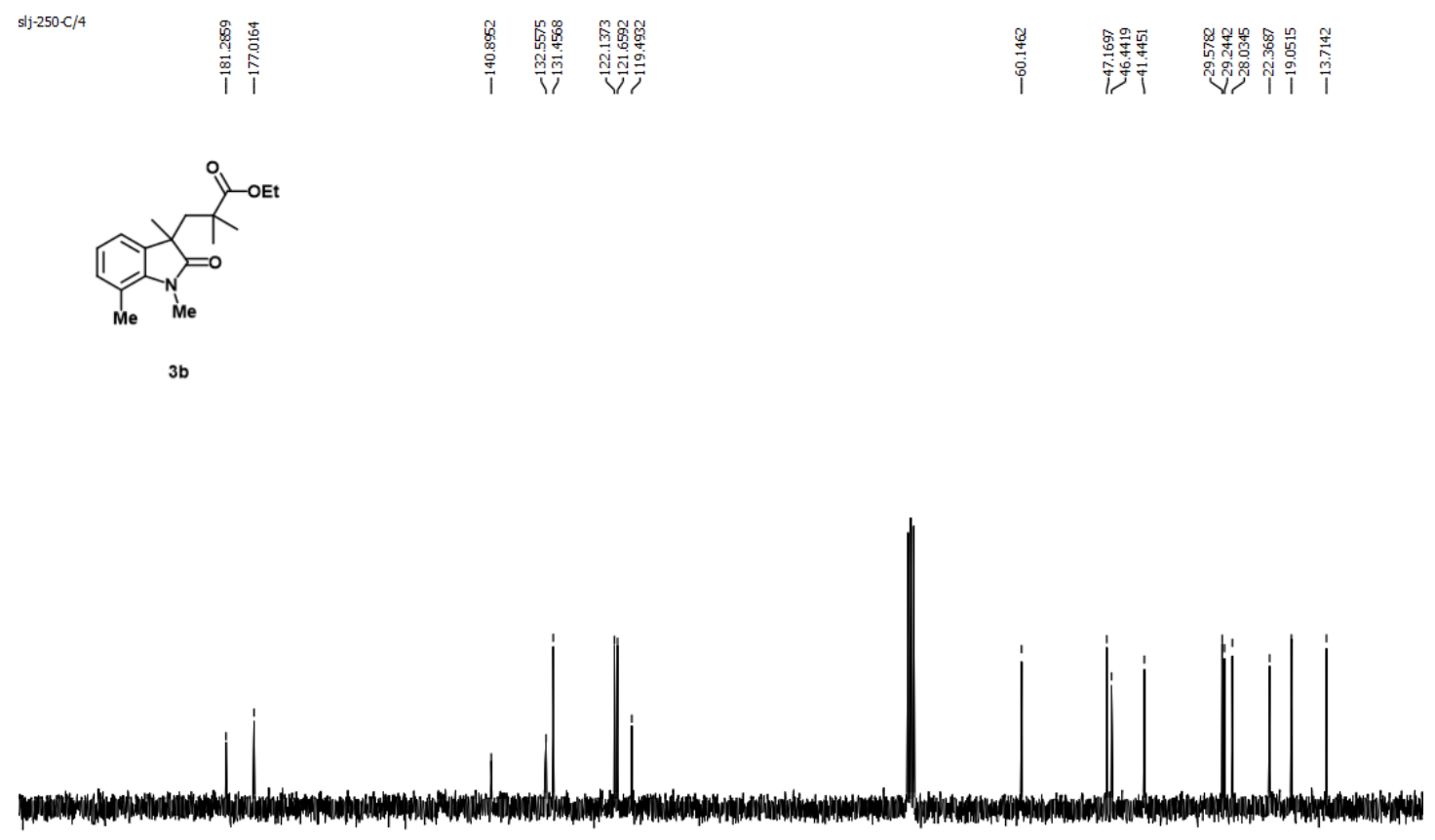

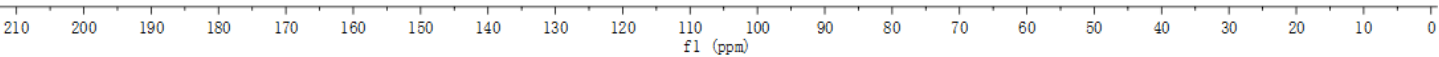


${ }^{1} \mathbf{H}$ NMR of compound $\mathbf{3 C}$ and $\mathbf{3} \mathbf{C}^{\prime}$ (300 $\mathrm{MHz}$ in $\mathrm{CDCl}_{3}$ )

$s \mid j-186 \approx / 4$

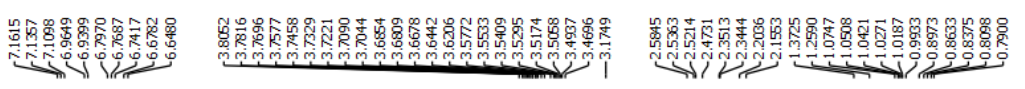
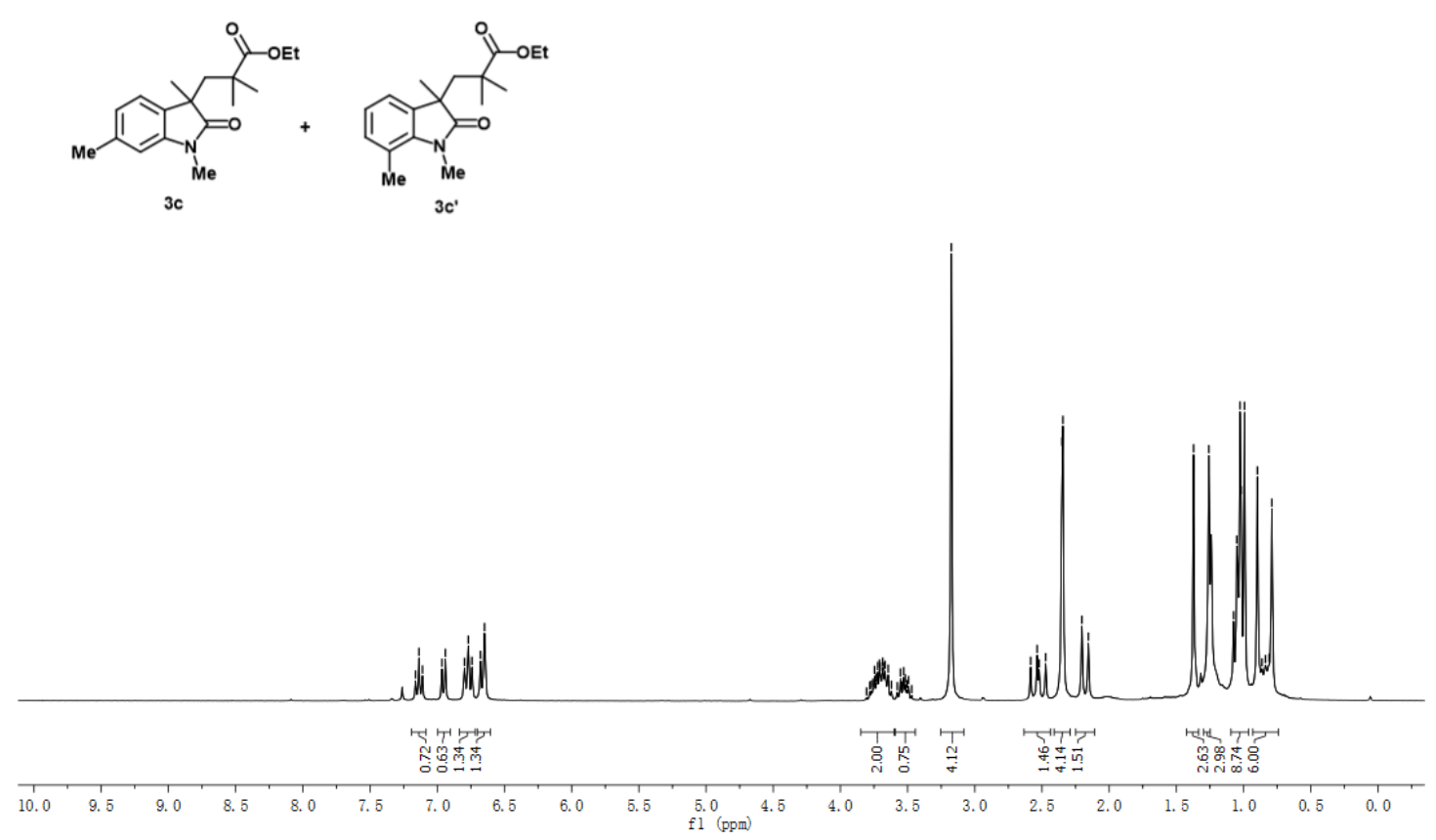

${ }^{13} \mathrm{C} \mathrm{NMR}$ of compound $\mathbf{3 C}$ and $\mathbf{3} \mathbf{C}^{\prime}\left(75 \mathrm{MHz}\right.$ in $\mathrm{CDCl}_{3}$ )

$s \mid j-186-\mathrm{a}-\mathrm{C} / 4$
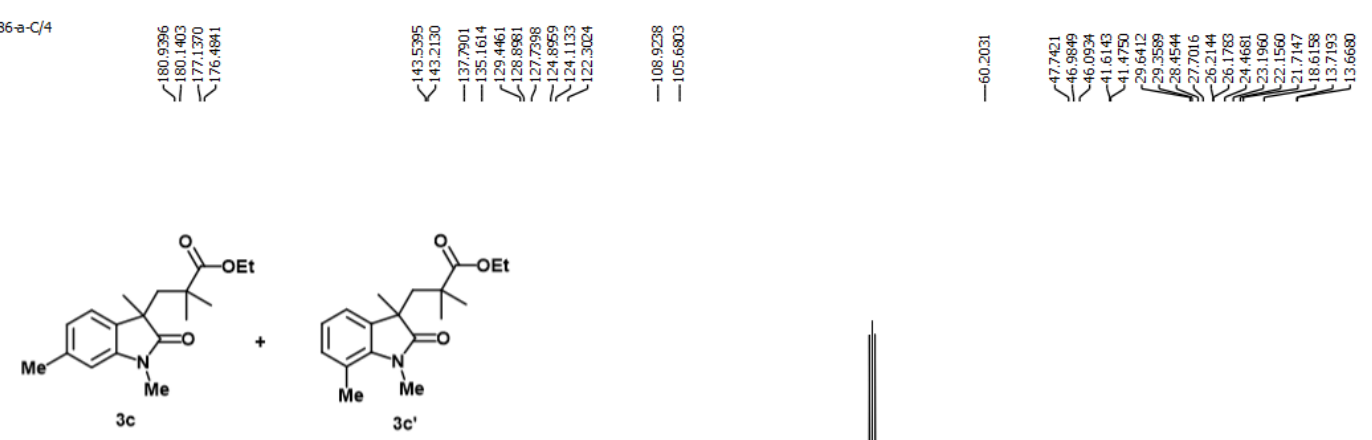

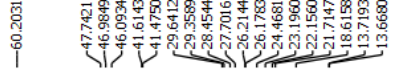

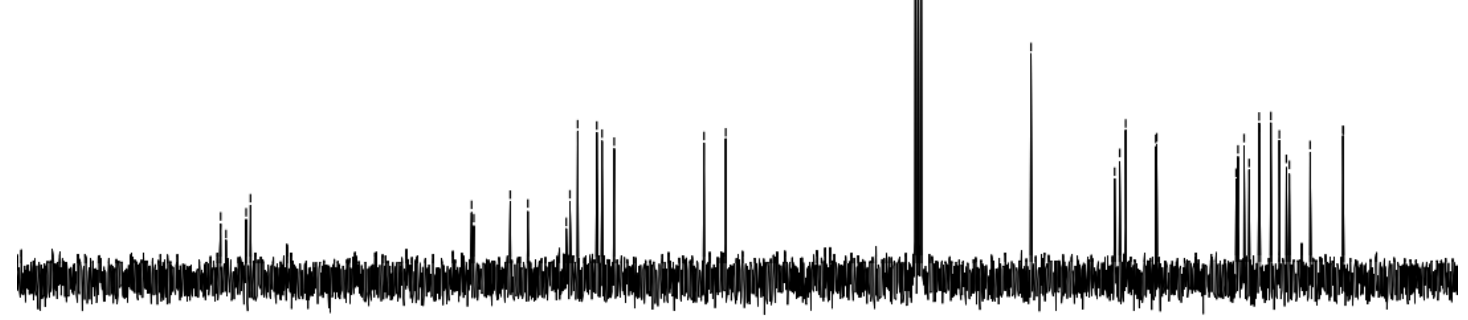

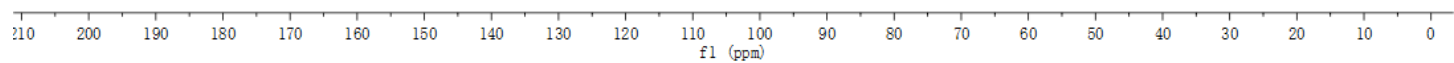


${ }^{1} \mathbf{H}$ NMR of compound $\mathbf{3 d}\left(300 \mathrm{MHz}\right.$ in $\left.\mathrm{CDCl}_{3}\right)$

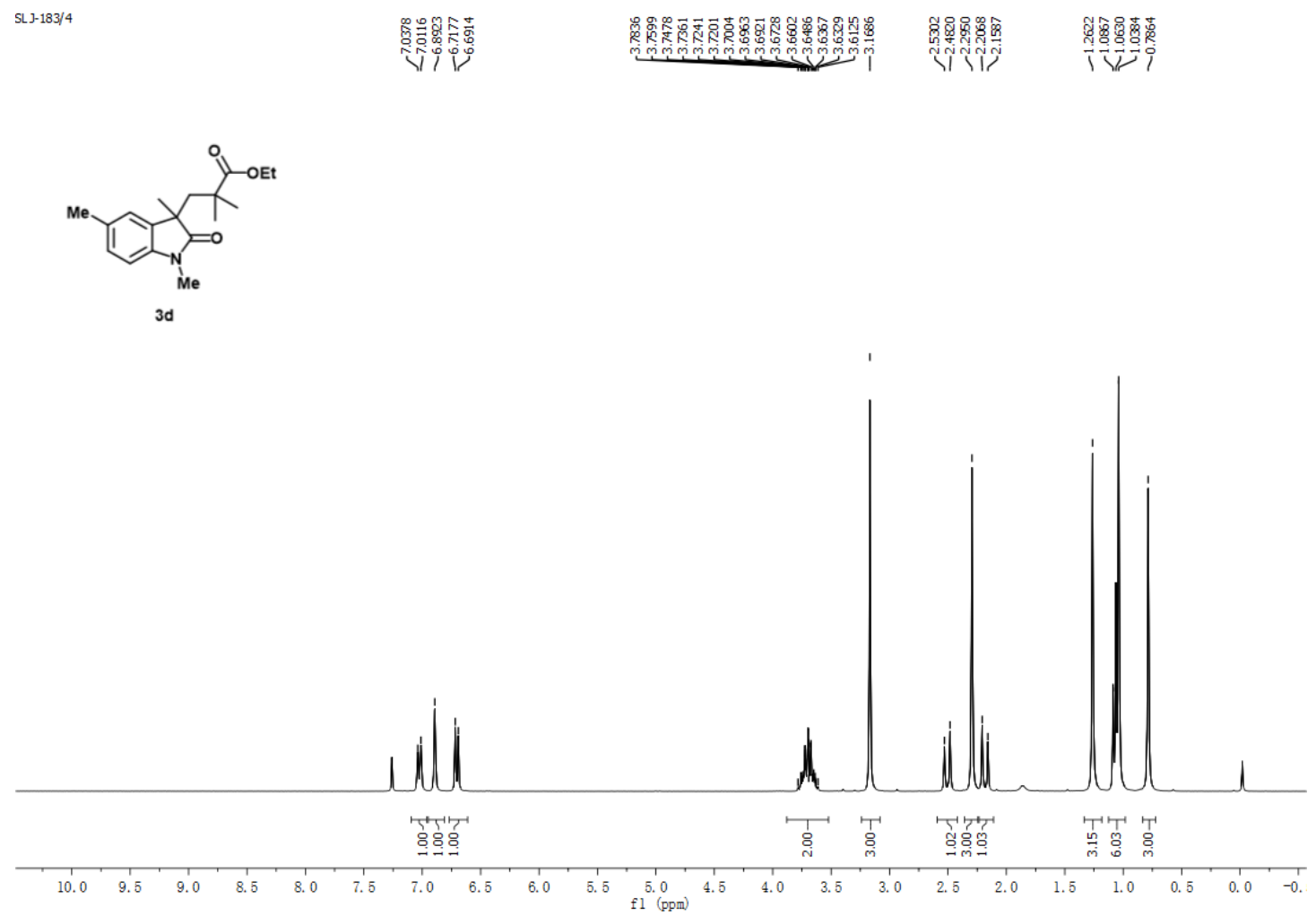

${ }^{13} \mathrm{C}$ NMR of compound $\mathbf{3 d}\left(75 \mathrm{MHz}\right.$ in $\left.\mathrm{CDCl}_{3}\right)$
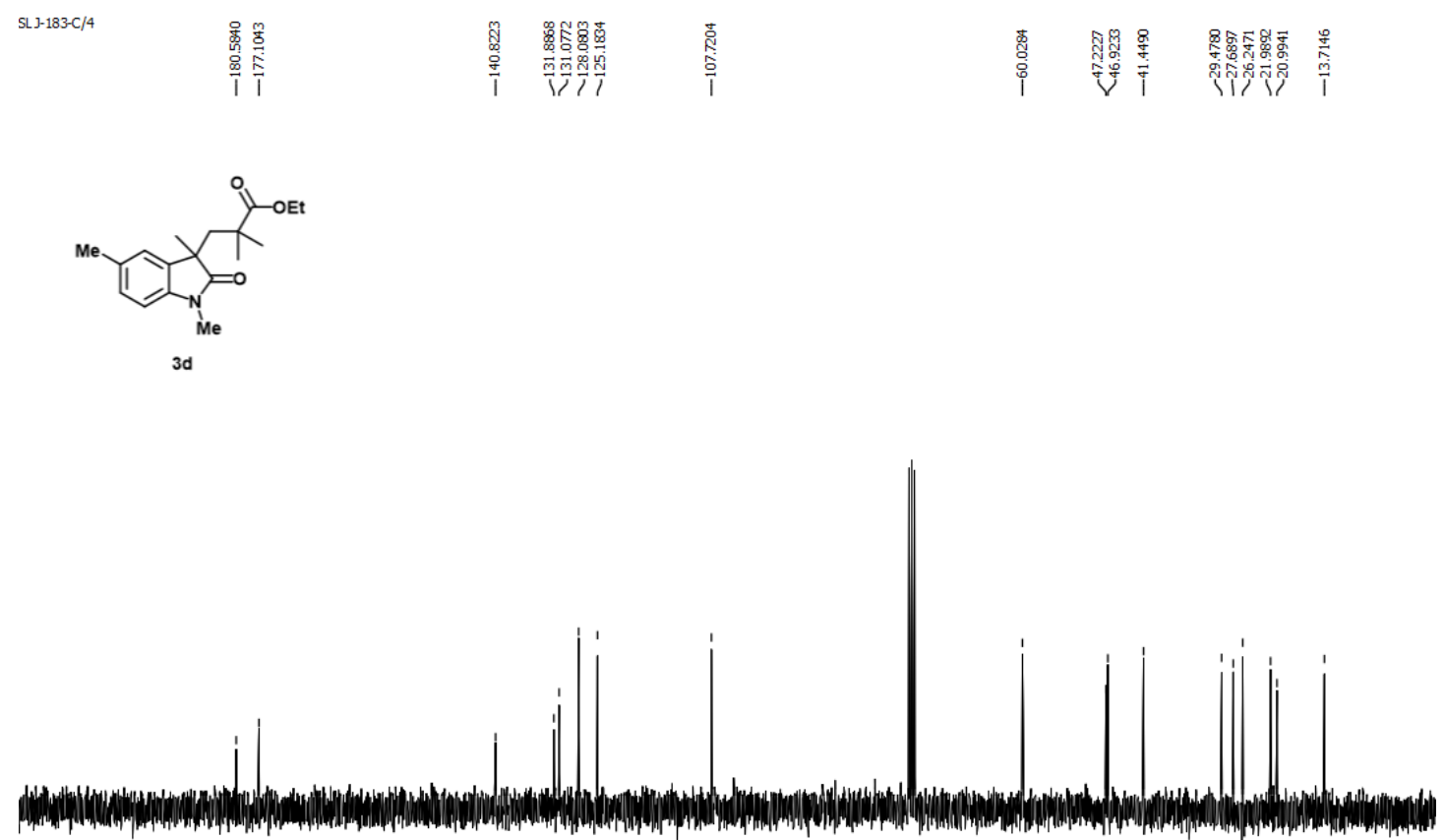

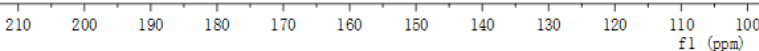


${ }^{1} \mathbf{H}$ NMR of compound $\mathbf{3 e}\left(300 \mathrm{MHz}\right.$ in $\left.\mathrm{CDCl}_{3}\right)$

$\mathrm{slj}-210 / 4$

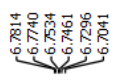

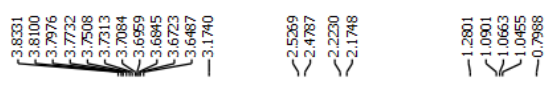

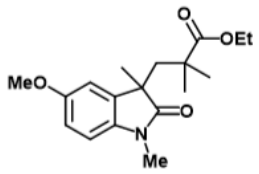

$3 e$

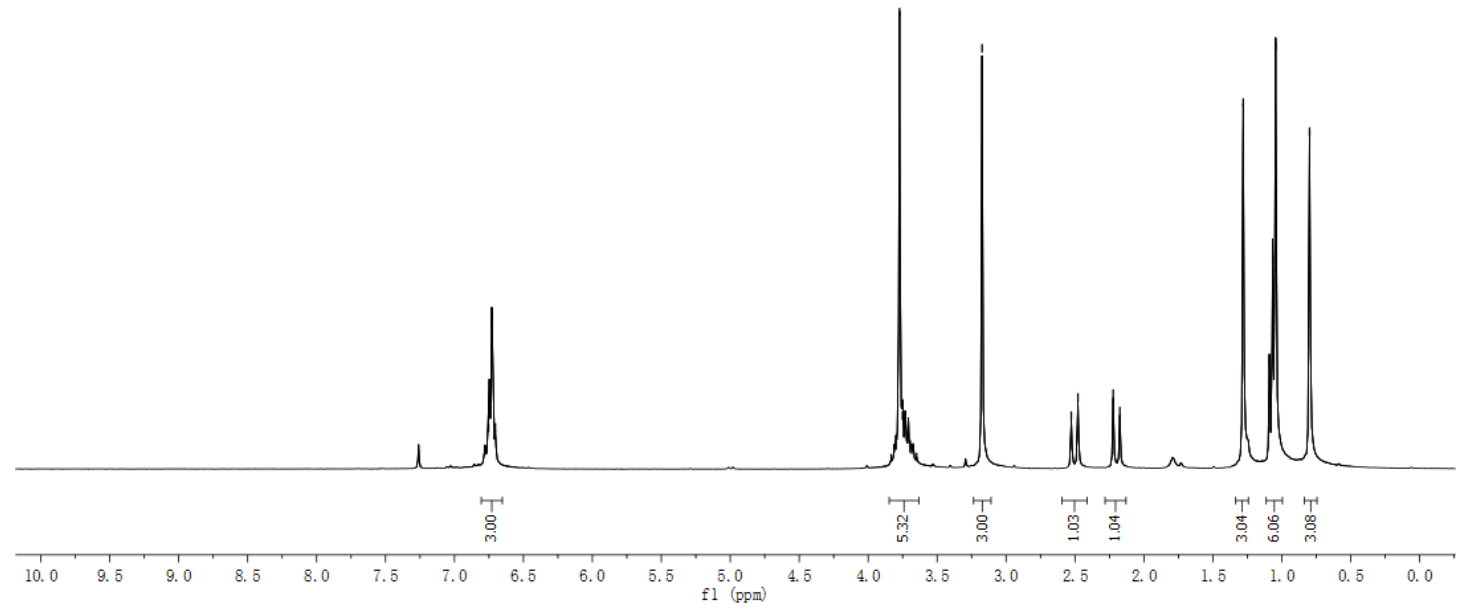

${ }^{13} \mathrm{C}$ NMR of compound $3 e\left(75 \mathrm{MHz}\right.$ in $\left.\mathrm{CDCl}_{3}\right)$

$\mathrm{sj} \mid-210 \mathrm{C} / 4$

$$
\text { IOEt }
$$

$3 e$
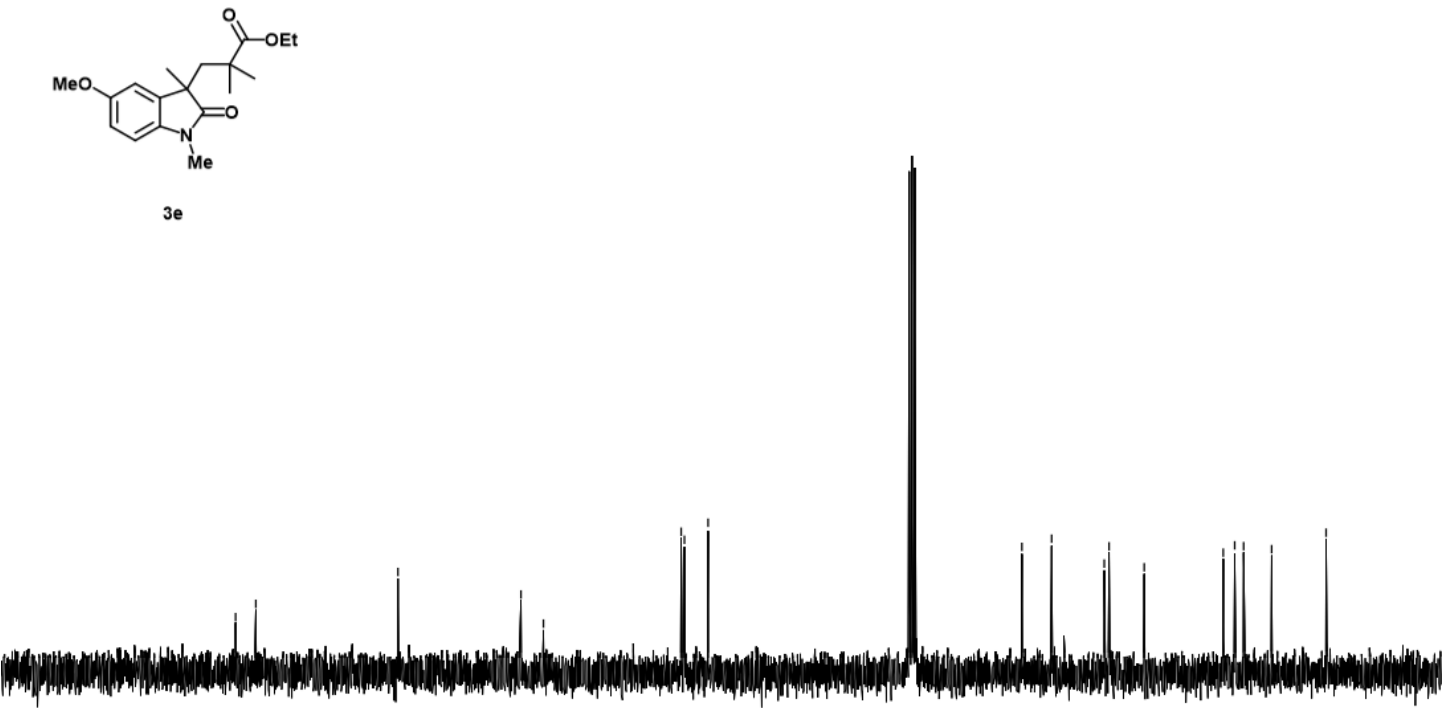

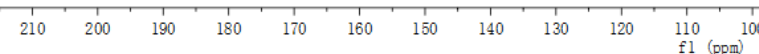


${ }^{1} \mathbf{H}$ NMR of compound $3 f\left(300 \mathrm{MHz}\right.$ in $\left.\mathrm{CDCl}_{3}\right)$
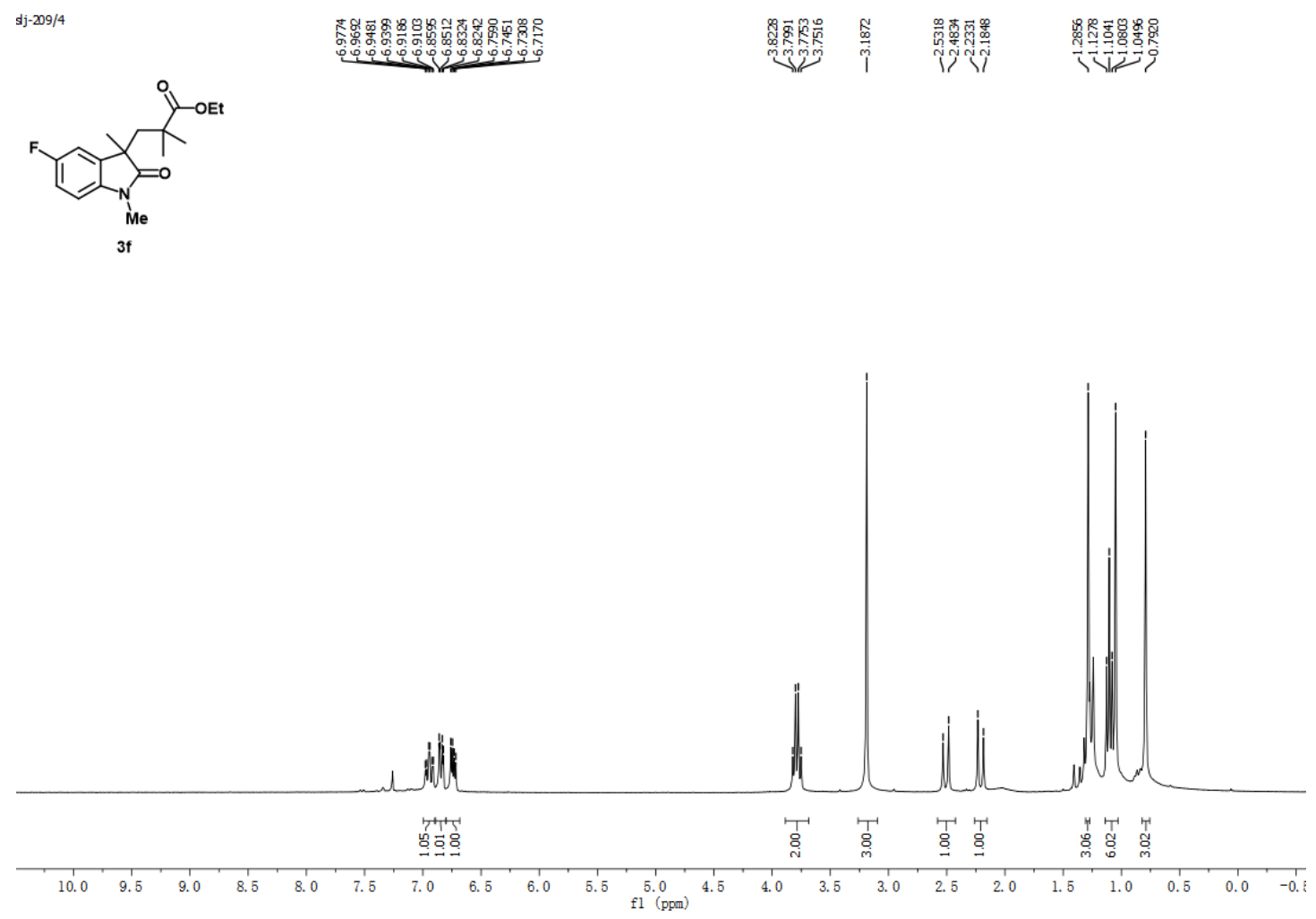

${ }^{13} \mathrm{C}$ NMR of compound $3 f\left(75 \mathrm{MHz}\right.$ in $\left.\mathrm{CDCl}_{3}\right)$

$s \mid j-209-C / 4$

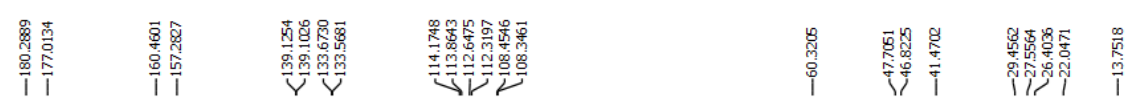

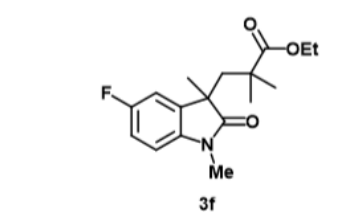

sf

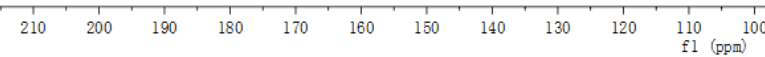


${ }^{19} \mathrm{~F}$ NMR of compound $\mathbf{3 f}\left(282 \mathrm{MHz}\right.$ in $\left.\mathrm{CDCl}_{3}\right)$

$25-209-F / 4$

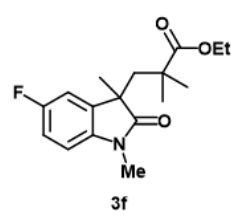

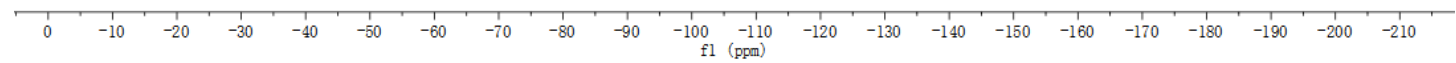

${ }^{1} \mathbf{H}$ NMR of compound $\mathbf{3 g}\left(300 \mathrm{MHz}\right.$ in $\left.\mathrm{CDCl}_{3}\right)$

$s \mid j-207 / 4$

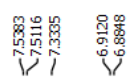

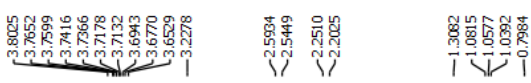<smiles></smiles>

$3 g$

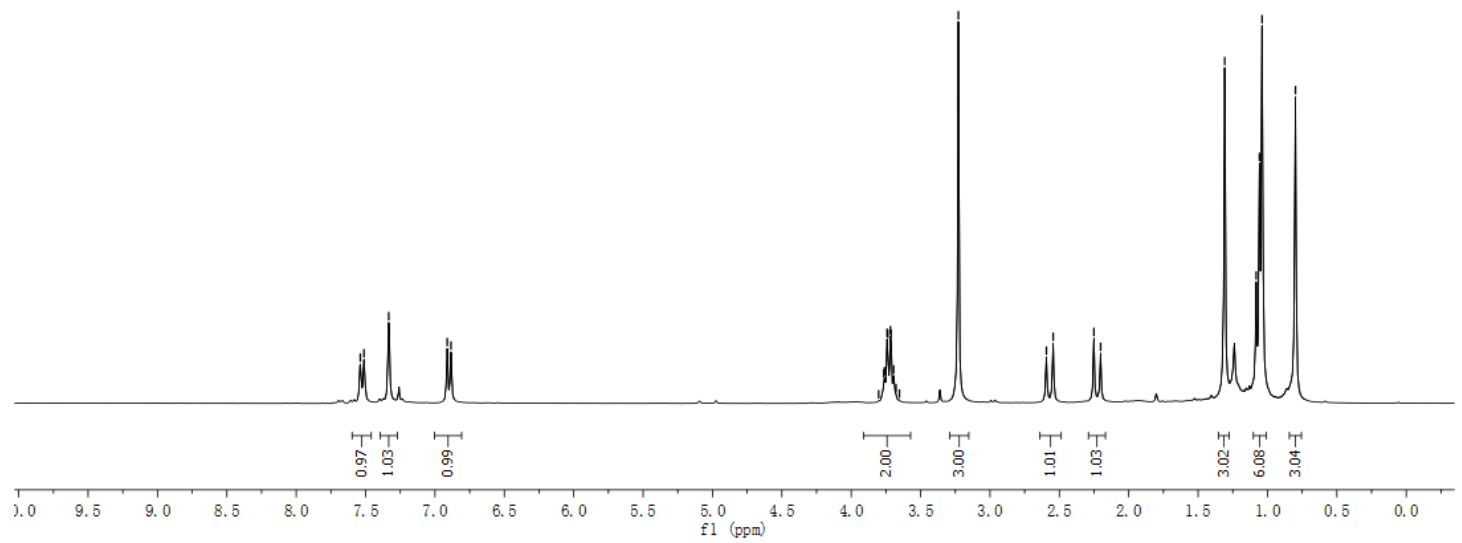


${ }^{13} \mathrm{C} \mathrm{NMR}$ of compound $\mathbf{3 g}$ (75 $\mathrm{MHz}$ in $\mathrm{CDCl}_{3}$ )
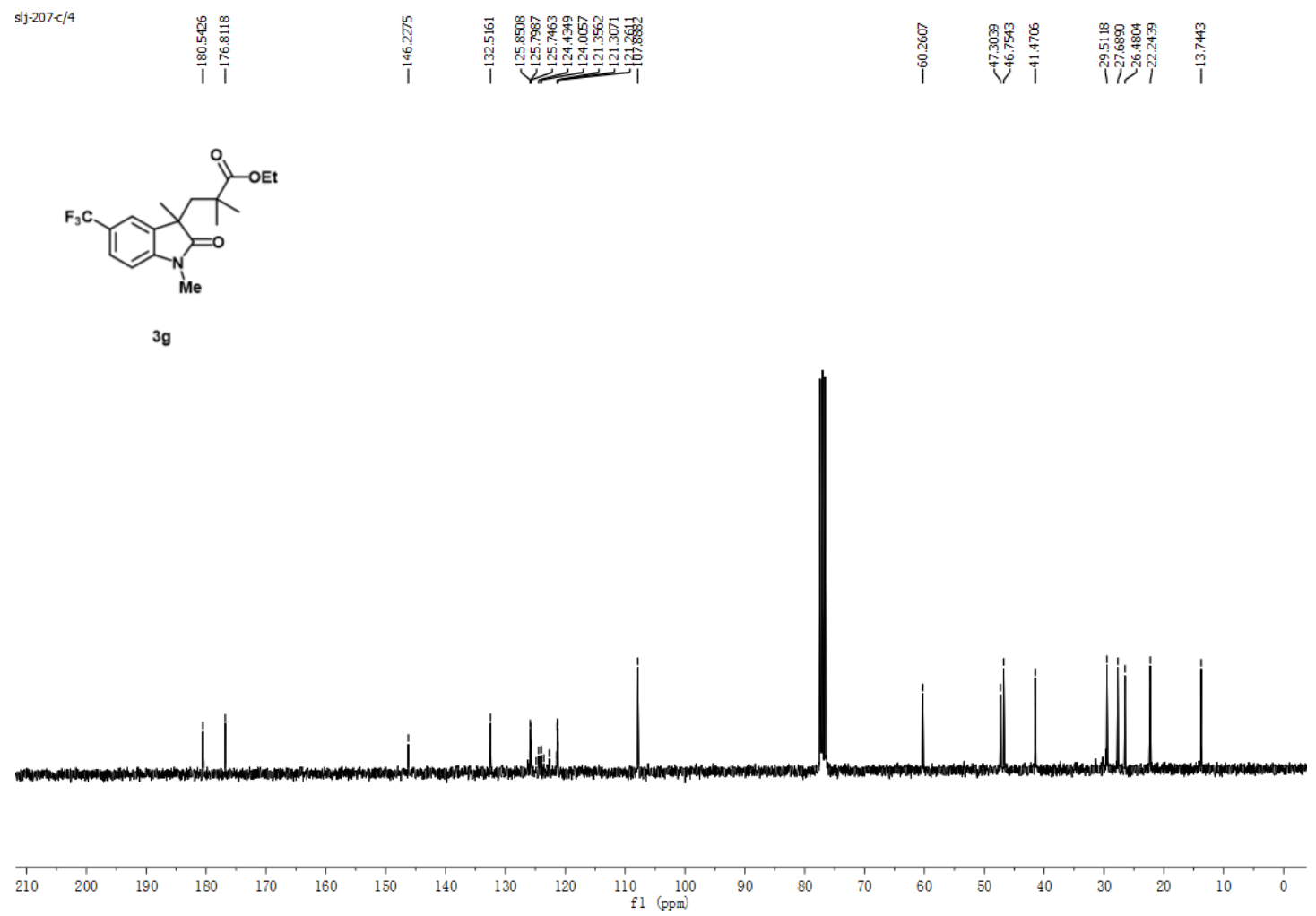

${ }^{19} \mathrm{~F}$ NMR of compound $\mathbf{3 g}$ (282 $\mathrm{MHz}$ in $\mathrm{CDCl}_{3}$ )

sj-207-F/2

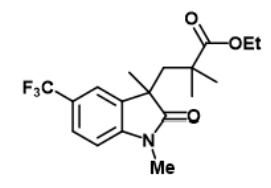

$3 \mathrm{~g}$

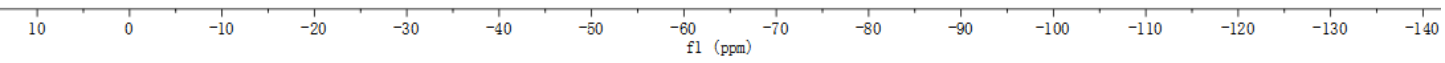


${ }^{1} \mathbf{H}$ NMR of compound $\mathbf{3 h}\left(300 \mathrm{MHz}\right.$ in $\left.\mathrm{CDCl}_{3}\right)$

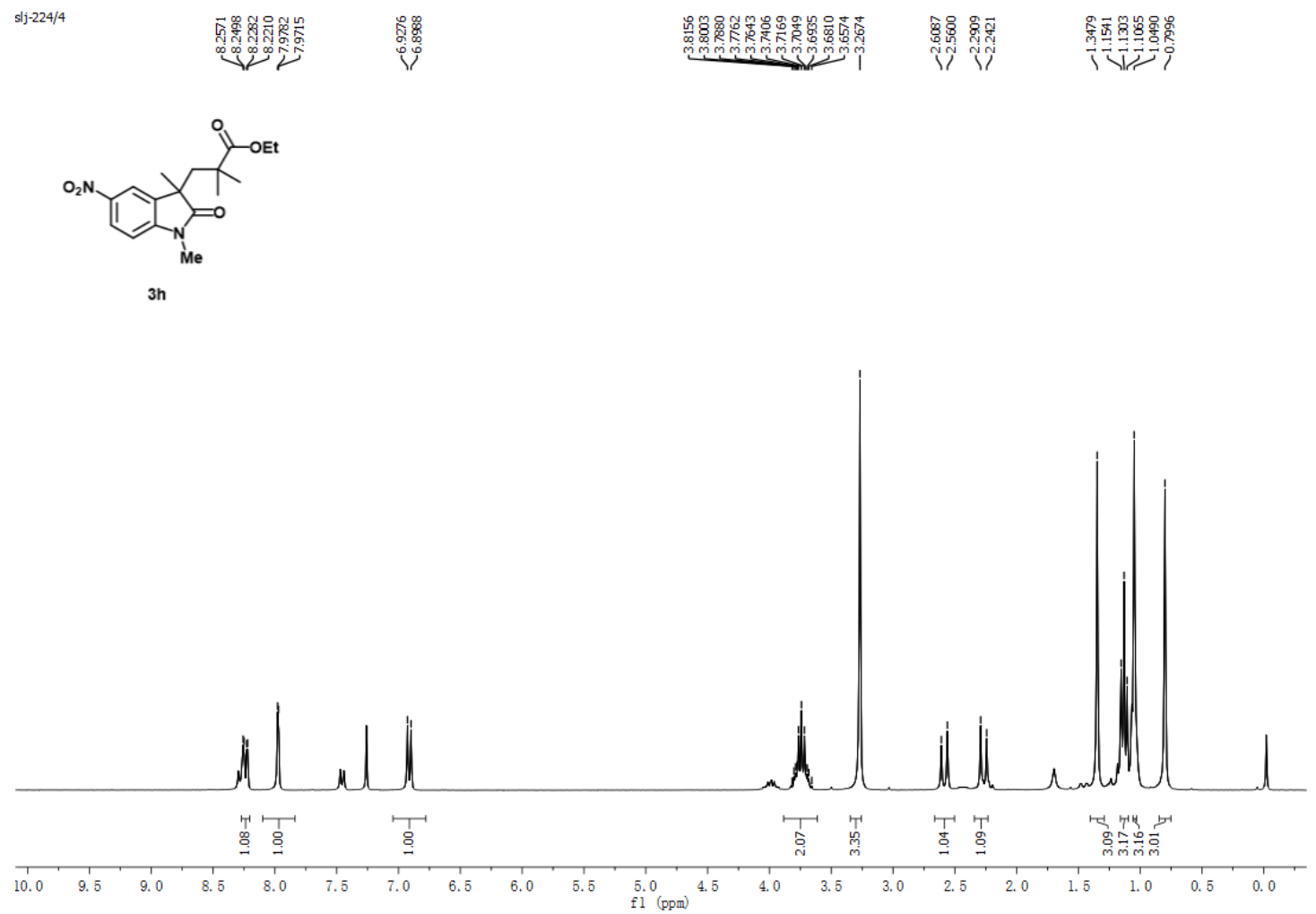

${ }^{13} \mathrm{C} \mathrm{NMR}$ of compound $3 \mathrm{~h}\left(75 \mathrm{MHz}\right.$ in $\left.\mathrm{CDCl}_{3}\right)$
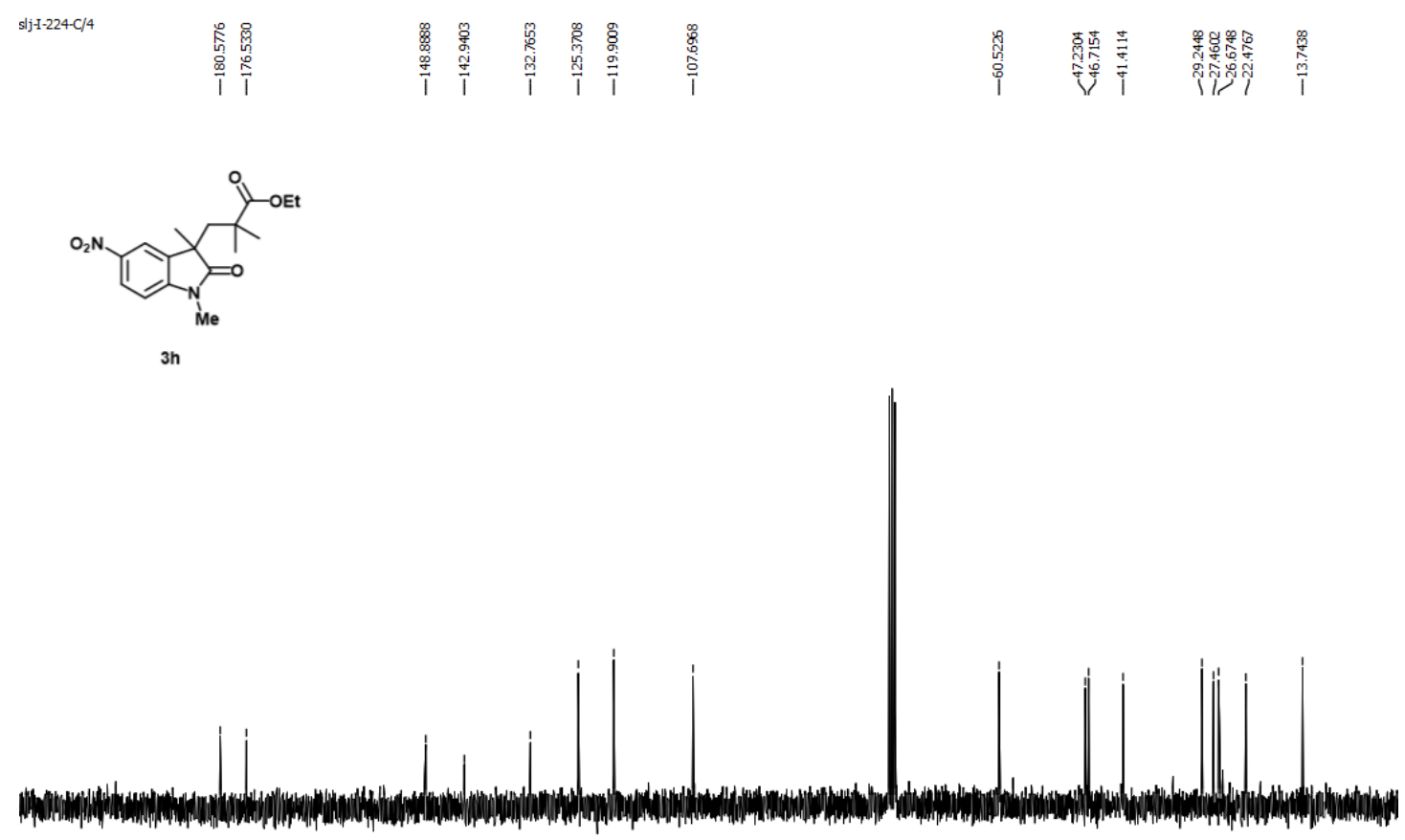

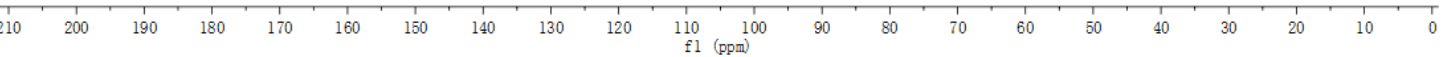


${ }^{1} \mathbf{H}$ NMR of compound $\mathbf{3 i}\left(300 \mathrm{MHz}\right.$ in $\mathrm{CDCl}_{3}$ )

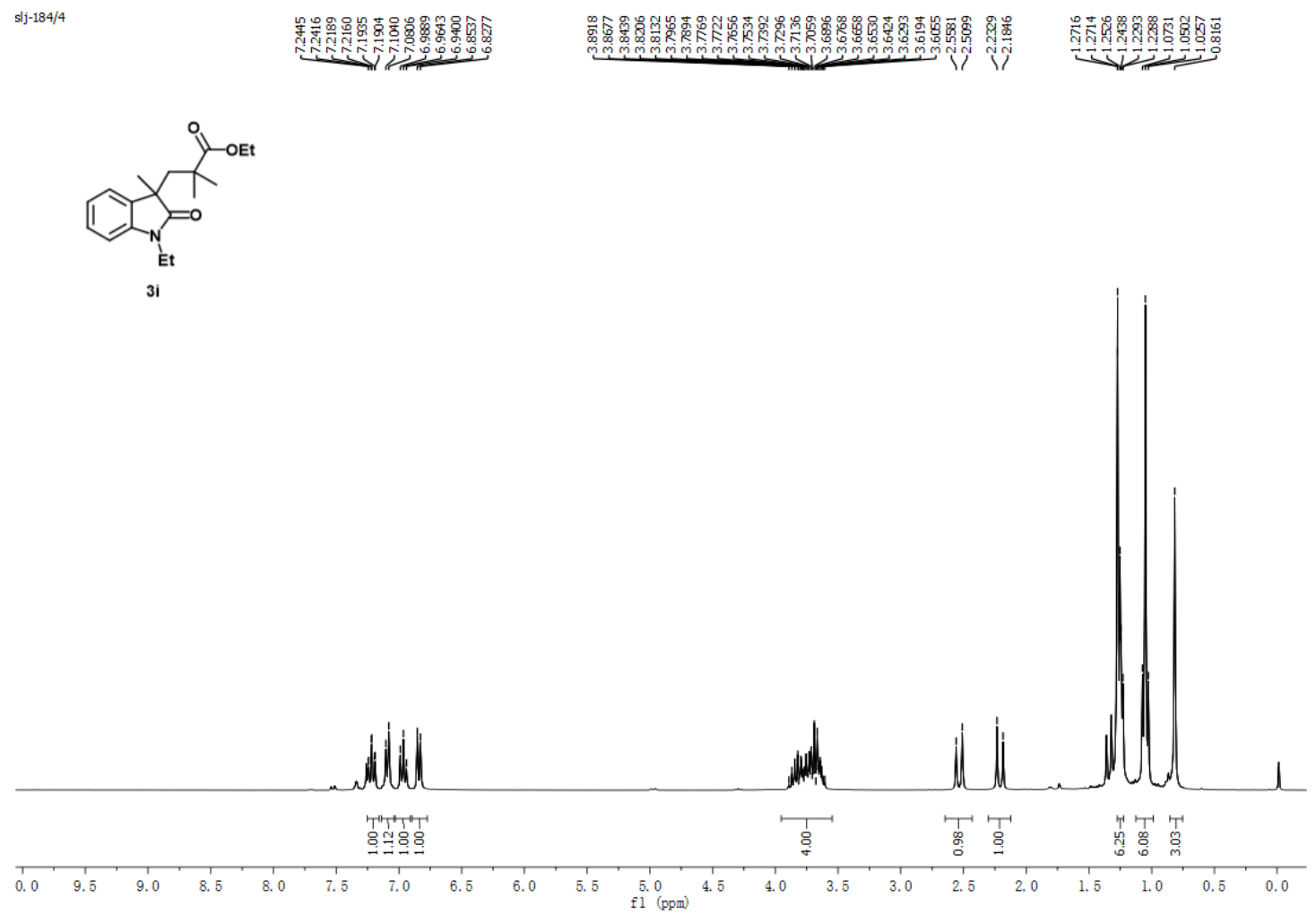

${ }^{13} \mathrm{C}$ NMR of compound $3 \mathbf{i}\left(75 \mathrm{MHz}\right.$ in $\mathrm{CDCl}_{3}$ )
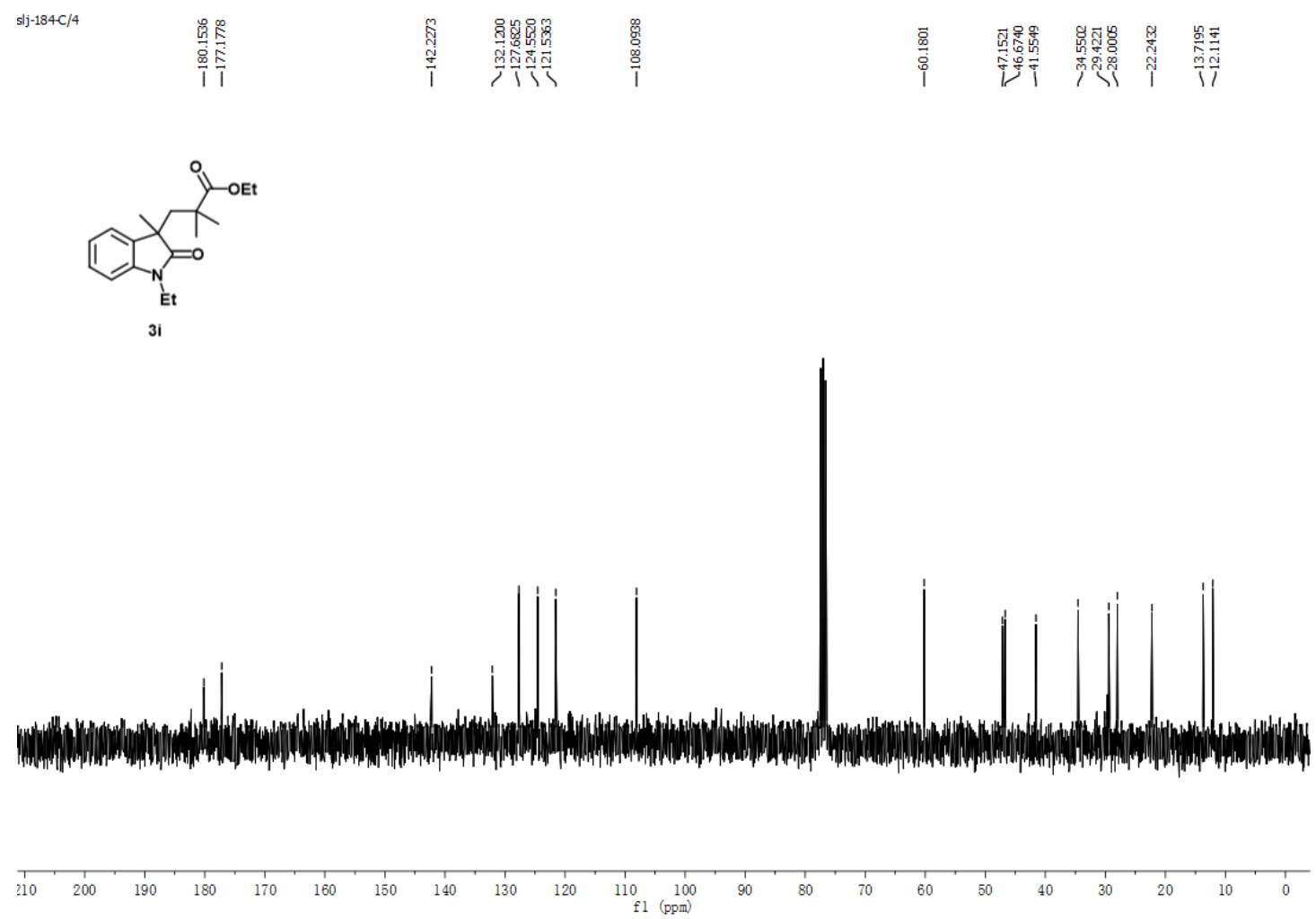
${ }^{1} \mathbf{H}$ NMR of compound $\mathbf{3 j}\left(300 \mathrm{MHz}\right.$ in $\left.\mathrm{CDCl}_{3}\right)$
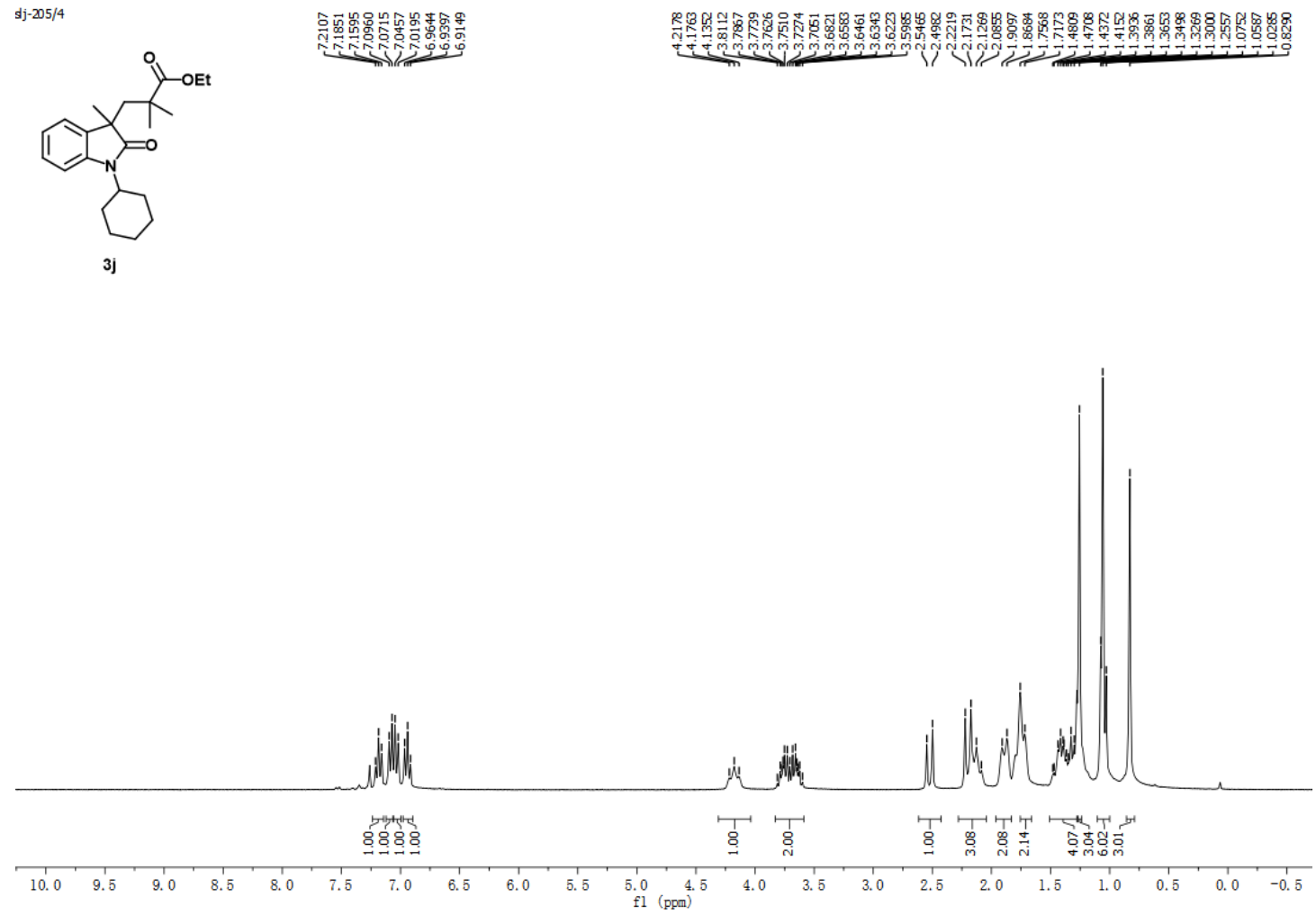

${ }^{13} \mathrm{C}$ NMR of compound $\mathbf{3} \mathbf{j}\left(75 \mathrm{MHz}\right.$ in $\mathrm{CDCl}_{3}$ )

slj-205-C/4

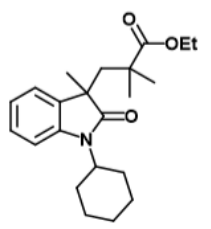

3j

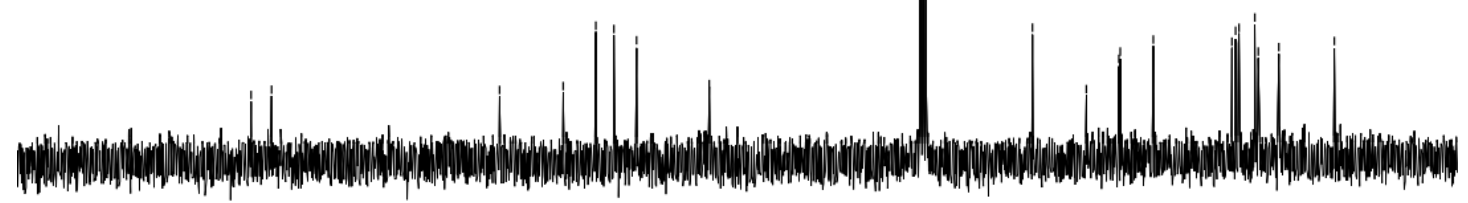

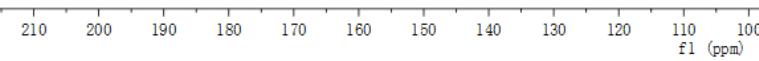


${ }^{1} \mathbf{H}$ NMR of compound $\mathbf{3 k}\left(300 \mathrm{MHz}\right.$ in $\mathrm{CDCl}_{3}$ )

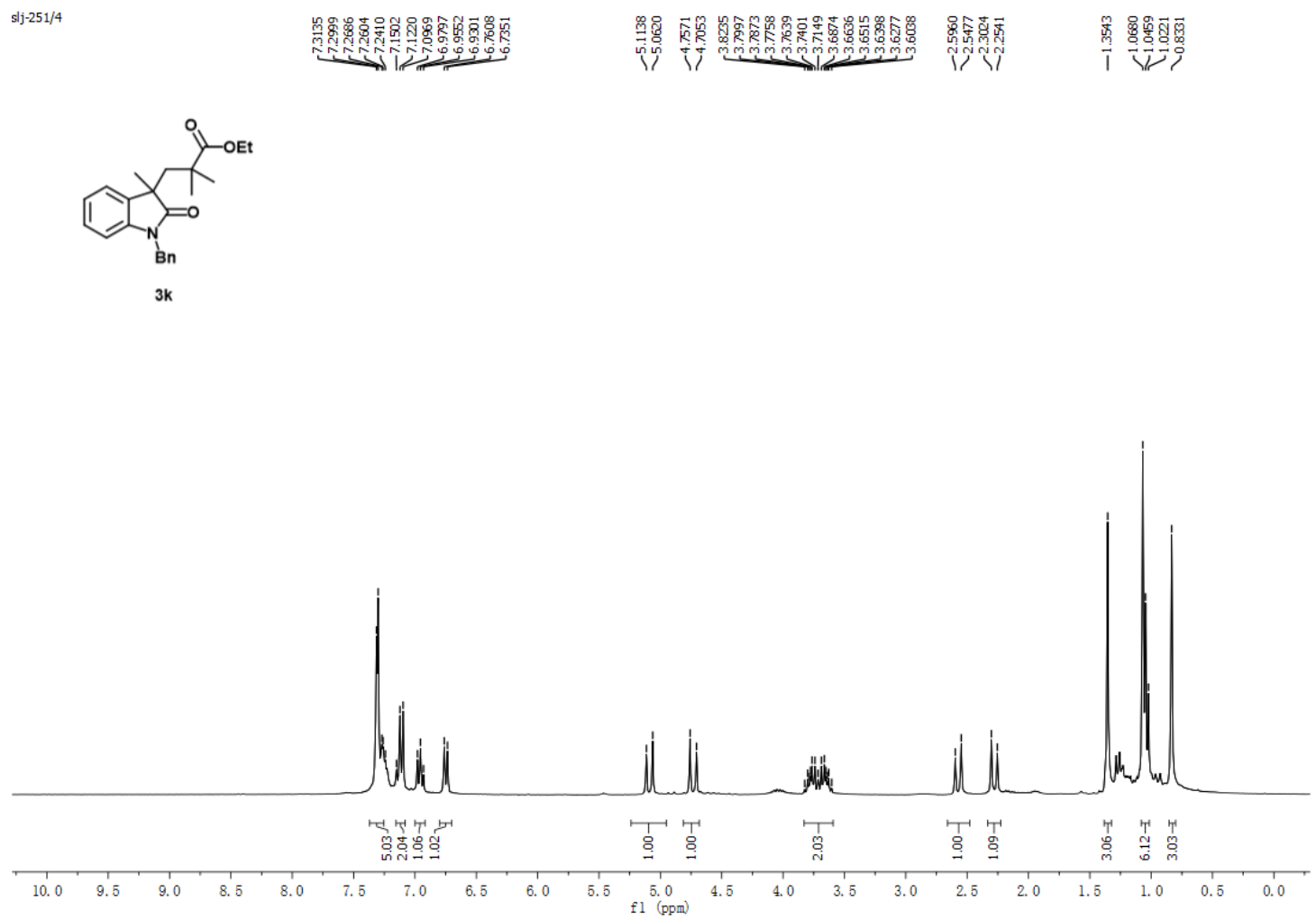

${ }^{13} \mathrm{C}$ NMR of compound $\mathbf{3 k}\left(75 \mathrm{MHz}\right.$ in $\mathrm{CDCl}_{3}$ )

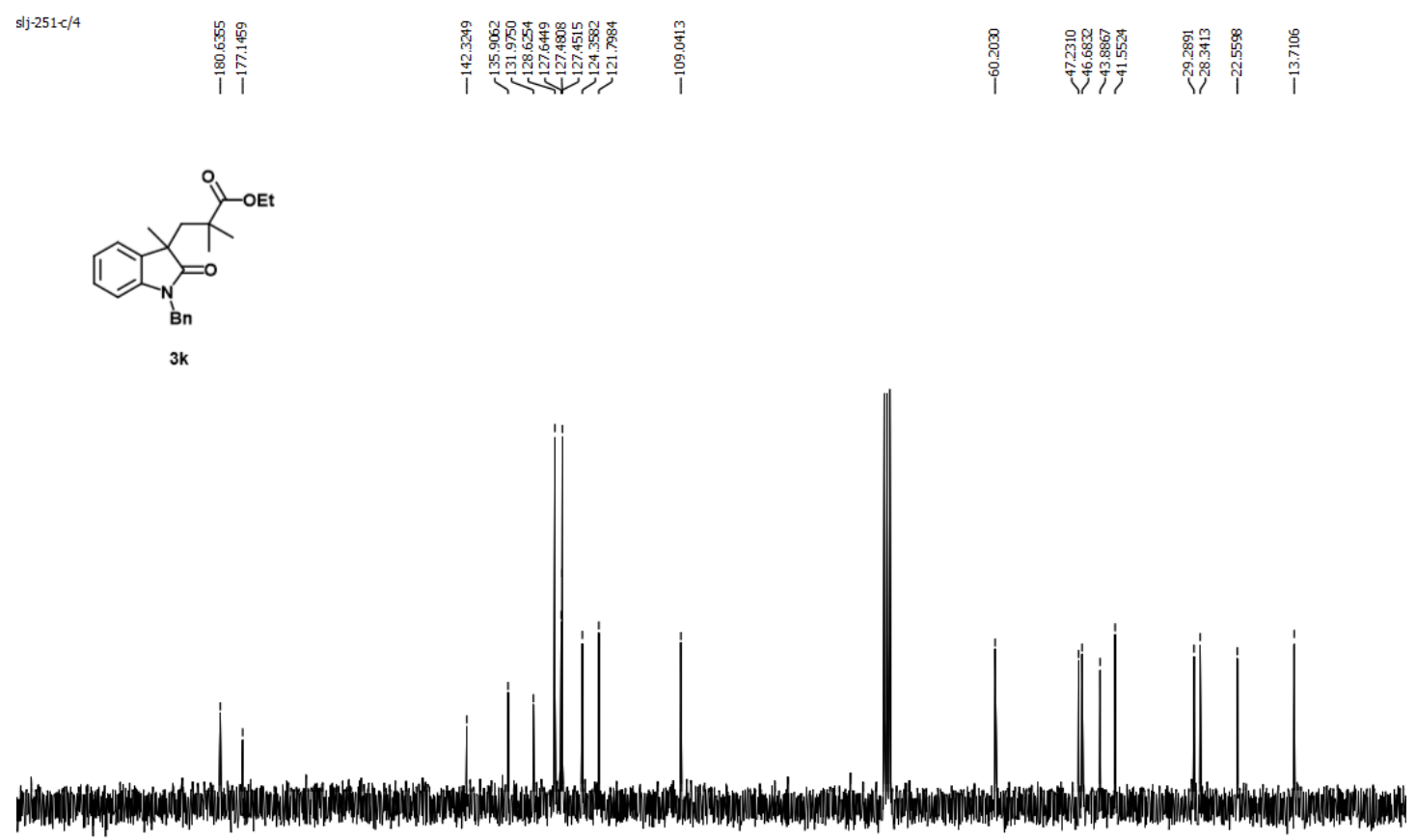

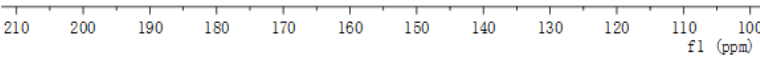


${ }^{1} \mathbf{H}$ NMR of compound $\mathbf{3 l}\left(300 \mathrm{MHz}\right.$ in $\left.\mathrm{CDCl}_{3}\right)$

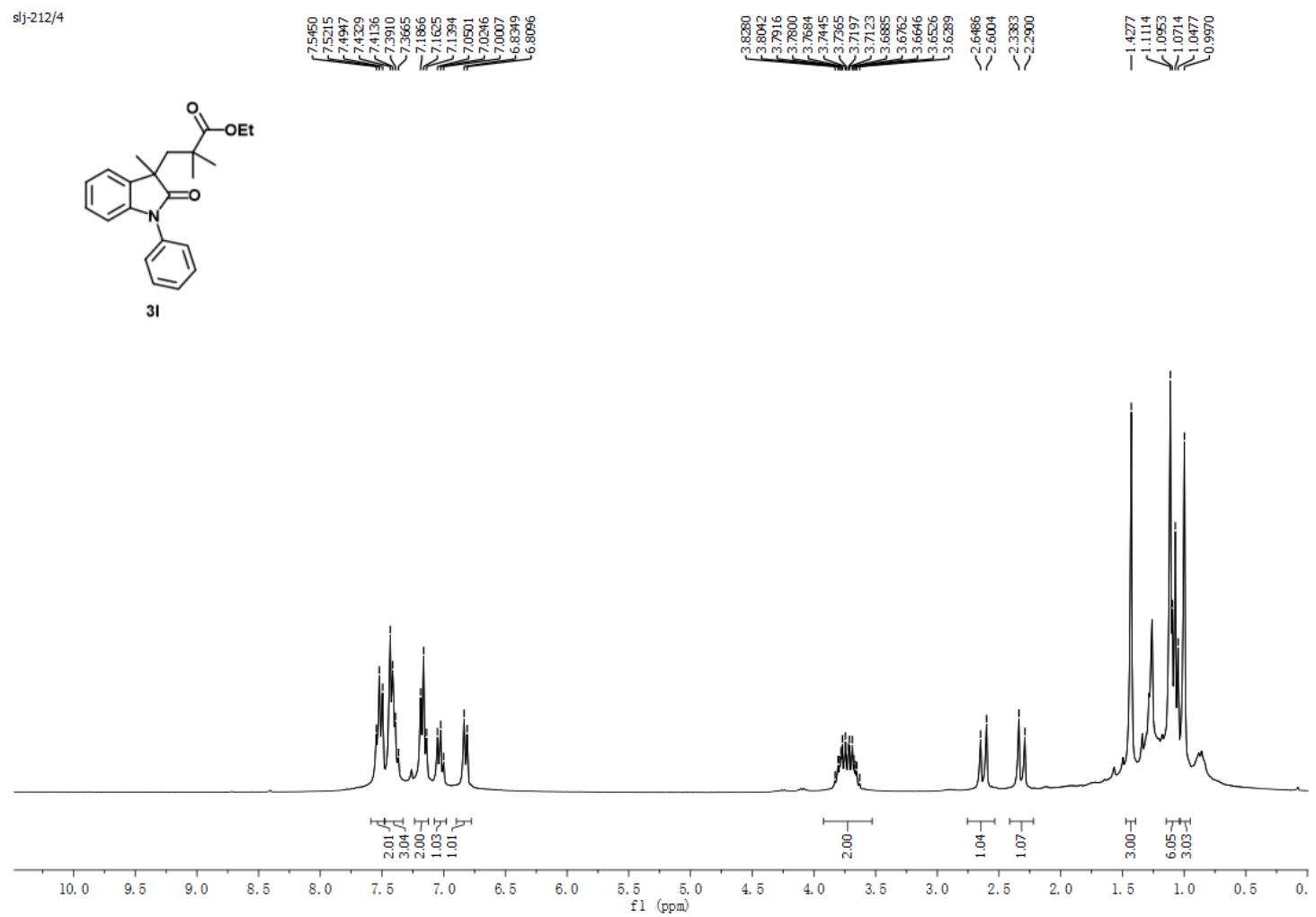

${ }^{13} \mathrm{C}$ NMR of compound $3 \mathbf{3}\left(75 \mathrm{MHz}\right.$ in $\mathrm{CDCl}_{3}$ )
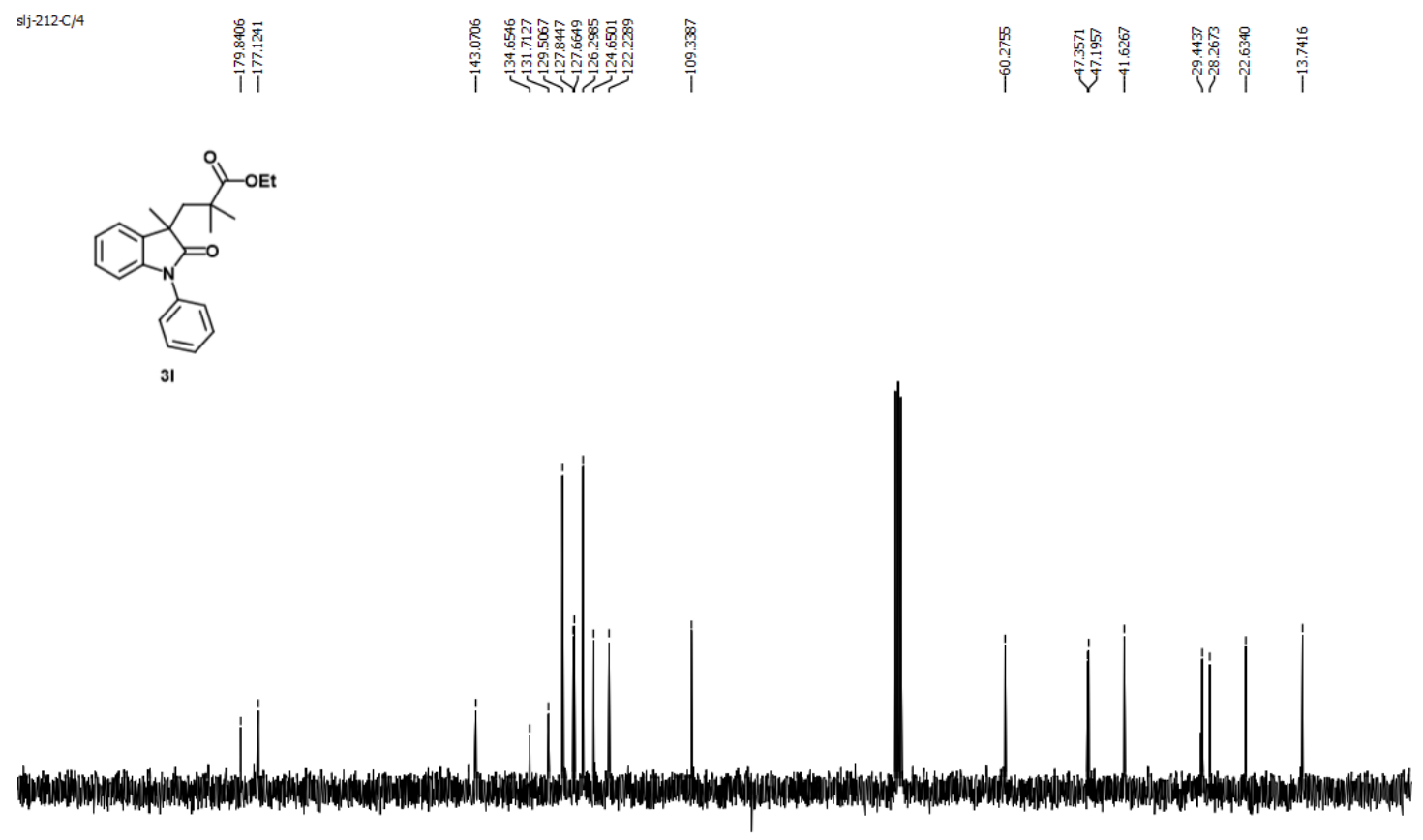

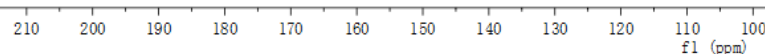


${ }^{1} \mathbf{H}$ NMR of compound $3 \mathrm{~m}\left(300 \mathrm{MHz}\right.$ in $\left.\mathrm{CDCl}_{3}\right)$

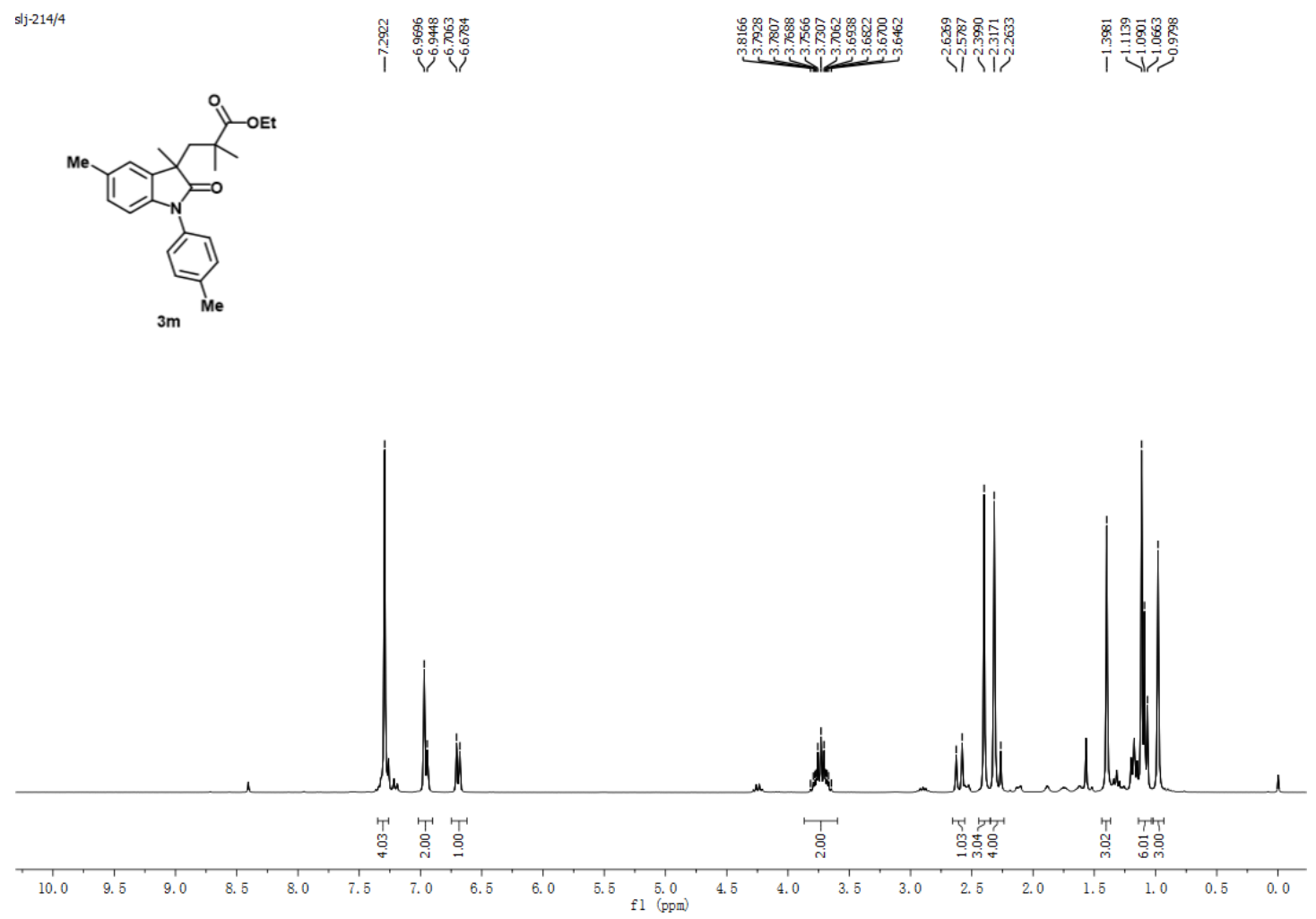

${ }^{13} \mathrm{C}$ NMR of compound $3 \mathrm{~m}\left(75 \mathrm{MHz}\right.$ in $\left.\mathrm{CDCl}_{3}\right)$
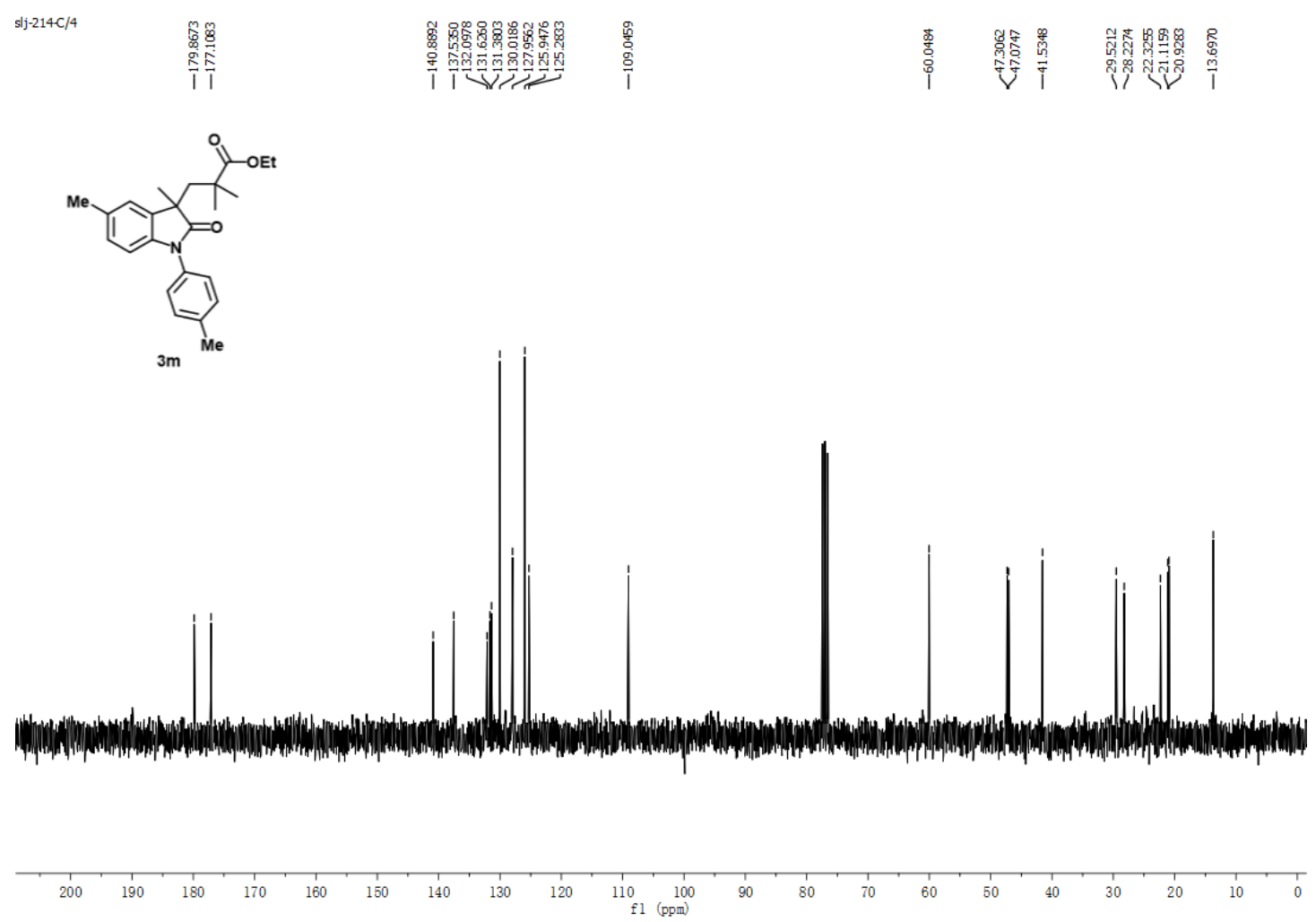
${ }^{1} \mathbf{H}$ NMR of compound $3 \mathbf{n}\left(300 \mathrm{MHz}\right.$ in $\left.\mathrm{CDCl}_{3}\right)$

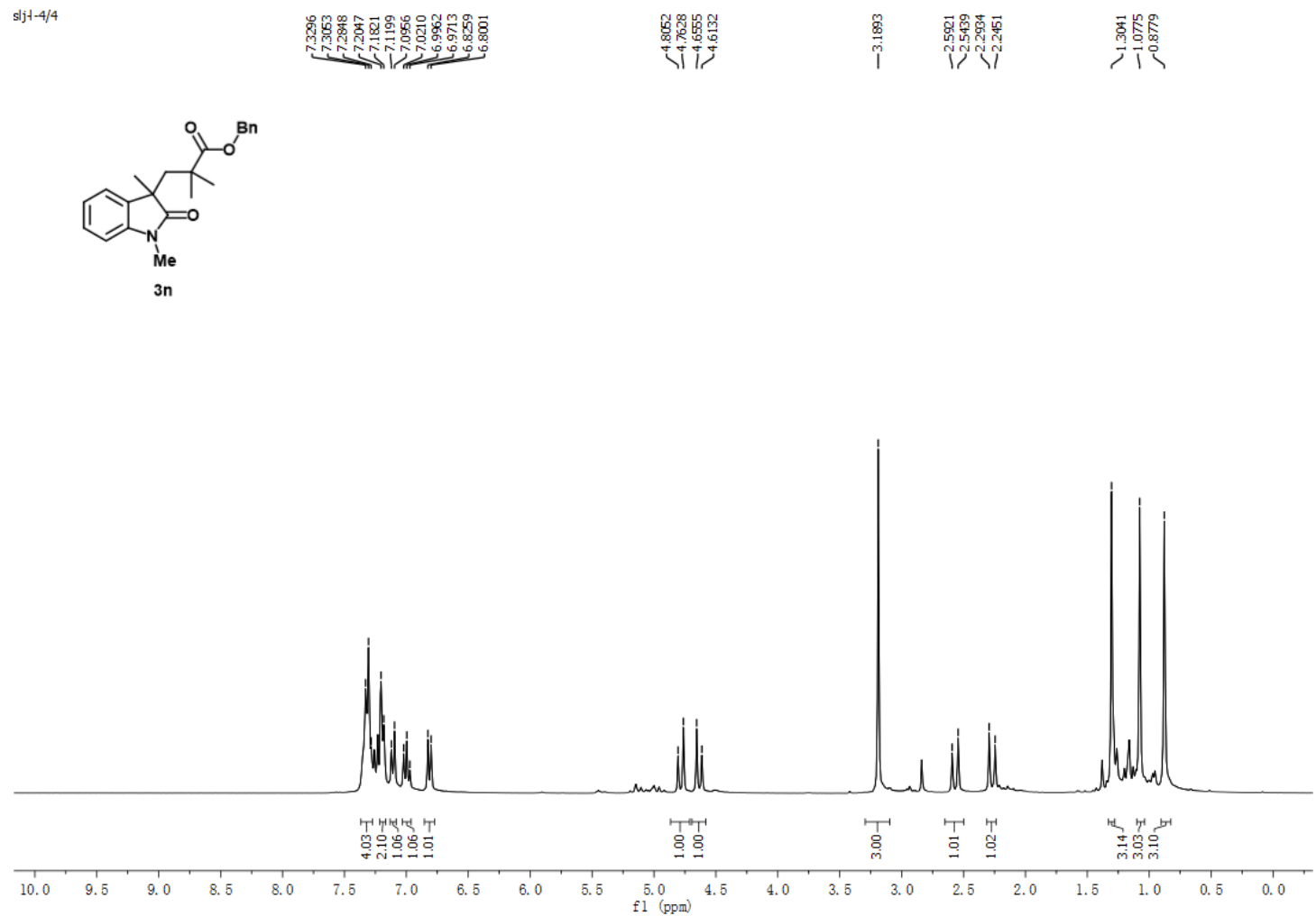

${ }^{13} \mathrm{C} \mathrm{NMR}$ of compound $3 \mathrm{n}\left(75 \mathrm{MHz}\right.$ in $\left.\mathrm{CDCl}_{3}\right)$

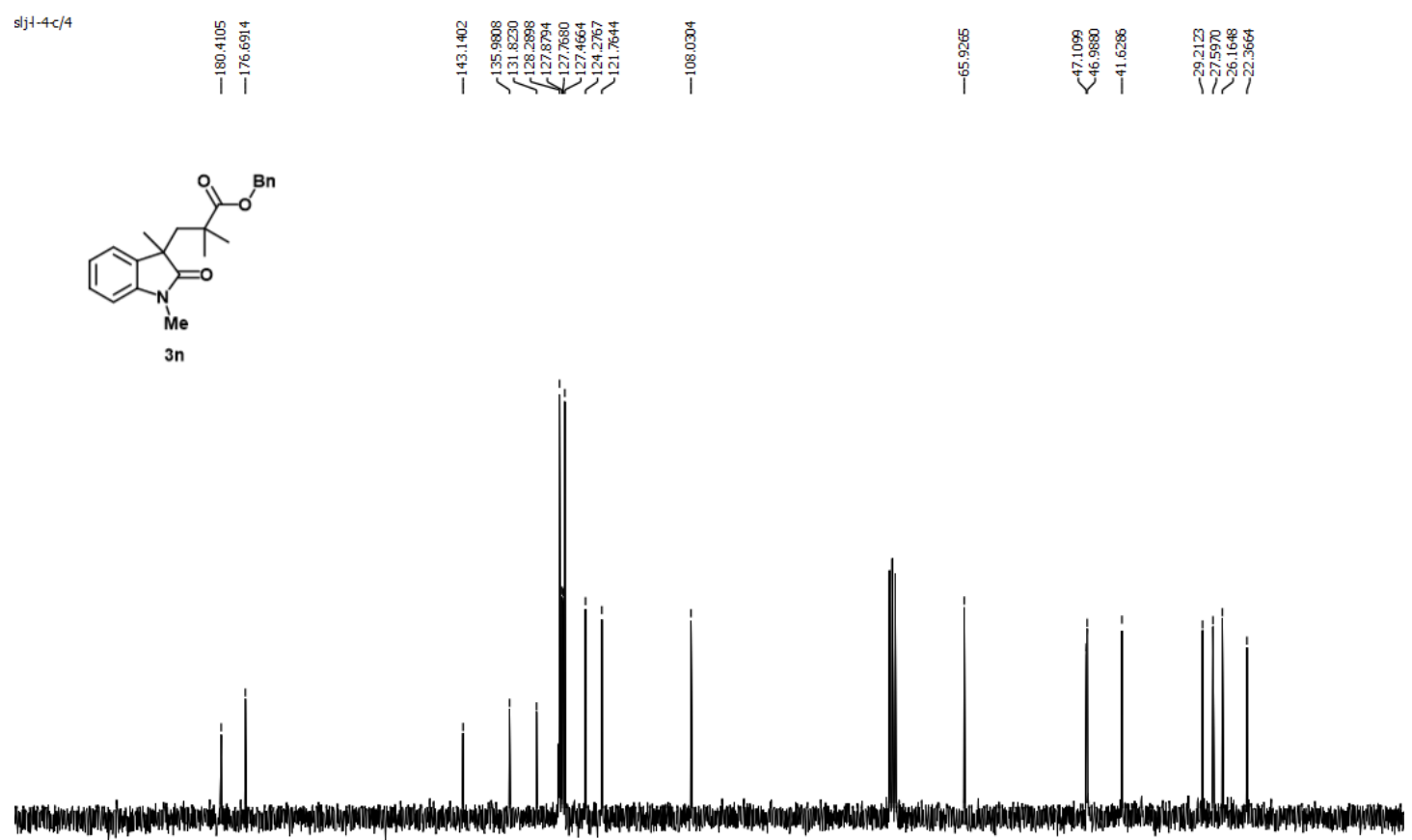

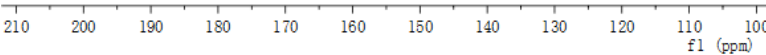


${ }^{1} \mathbf{H}$ NMR of compound $30\left(300 \mathrm{MHz}\right.$ in $\left.\mathrm{CDCl}_{3}\right)$

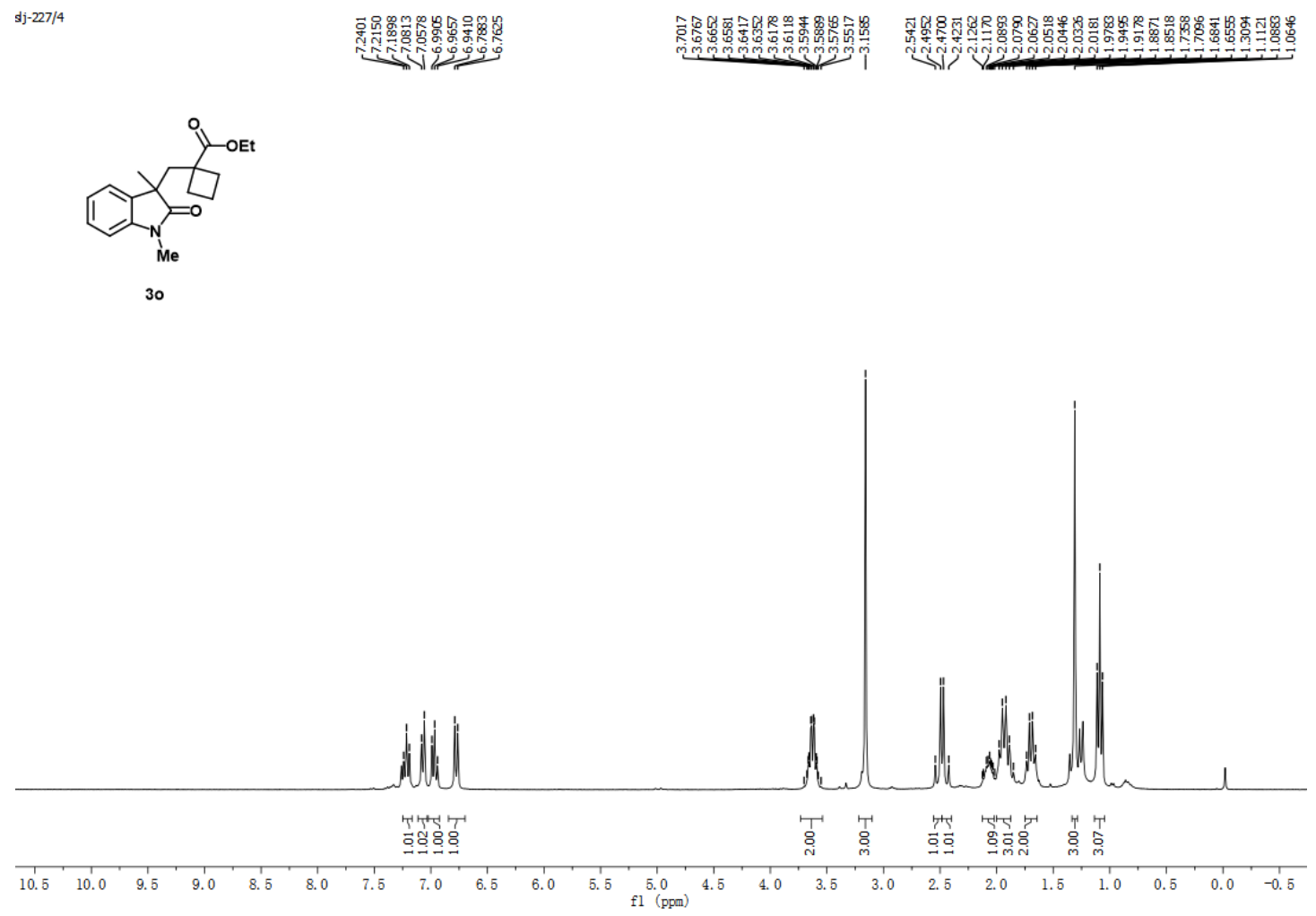

${ }^{13} \mathrm{C}$ NMR of compound $30\left(75 \mathrm{MHz}\right.$ in $\left.\mathrm{CDCl}_{3}\right)$

$s \mid j-227-C / 4$

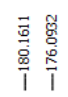

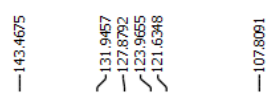

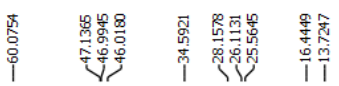

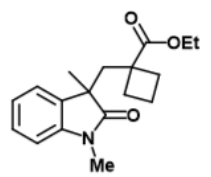

30

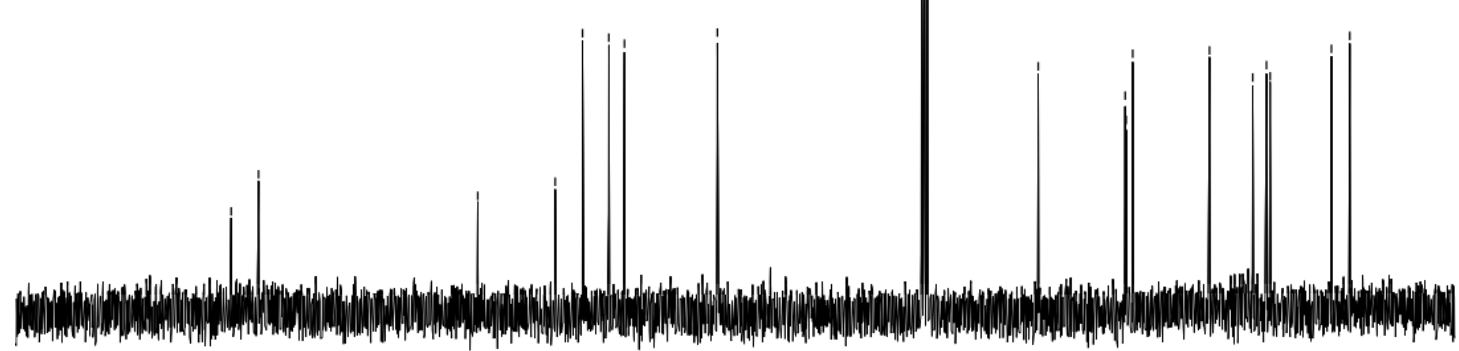

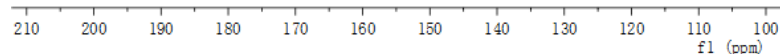


${ }^{1} \mathrm{H}$ NMR of compound $3 p\left(300 \mathrm{MHz}\right.$ in $\left.\mathrm{CDCl}_{3}\right)$

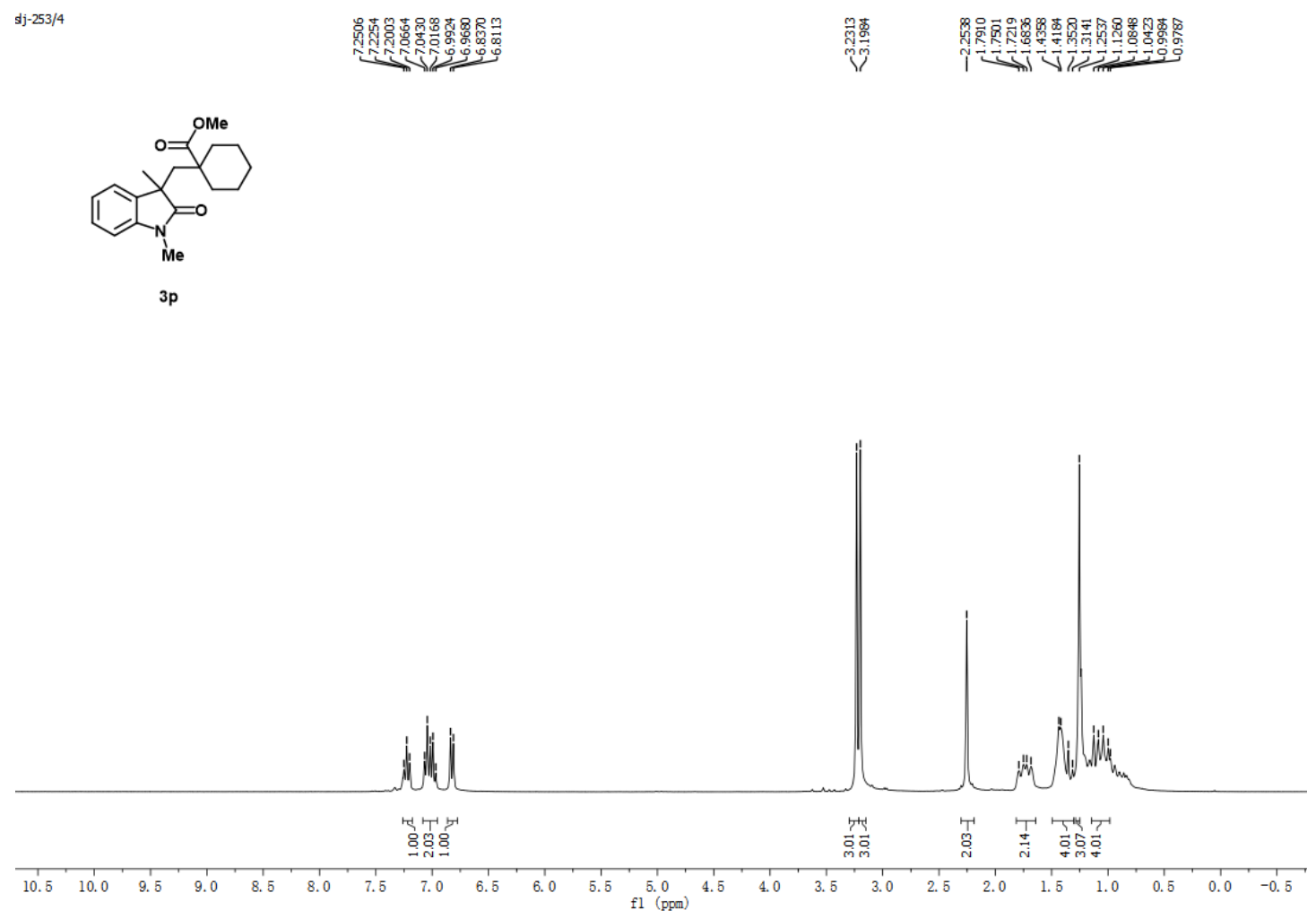

${ }^{13} \mathrm{C}$ NMR of compound $3 p\left(75 \mathrm{MHz}\right.$ in $\left.\mathrm{CDCl}_{3}\right)$

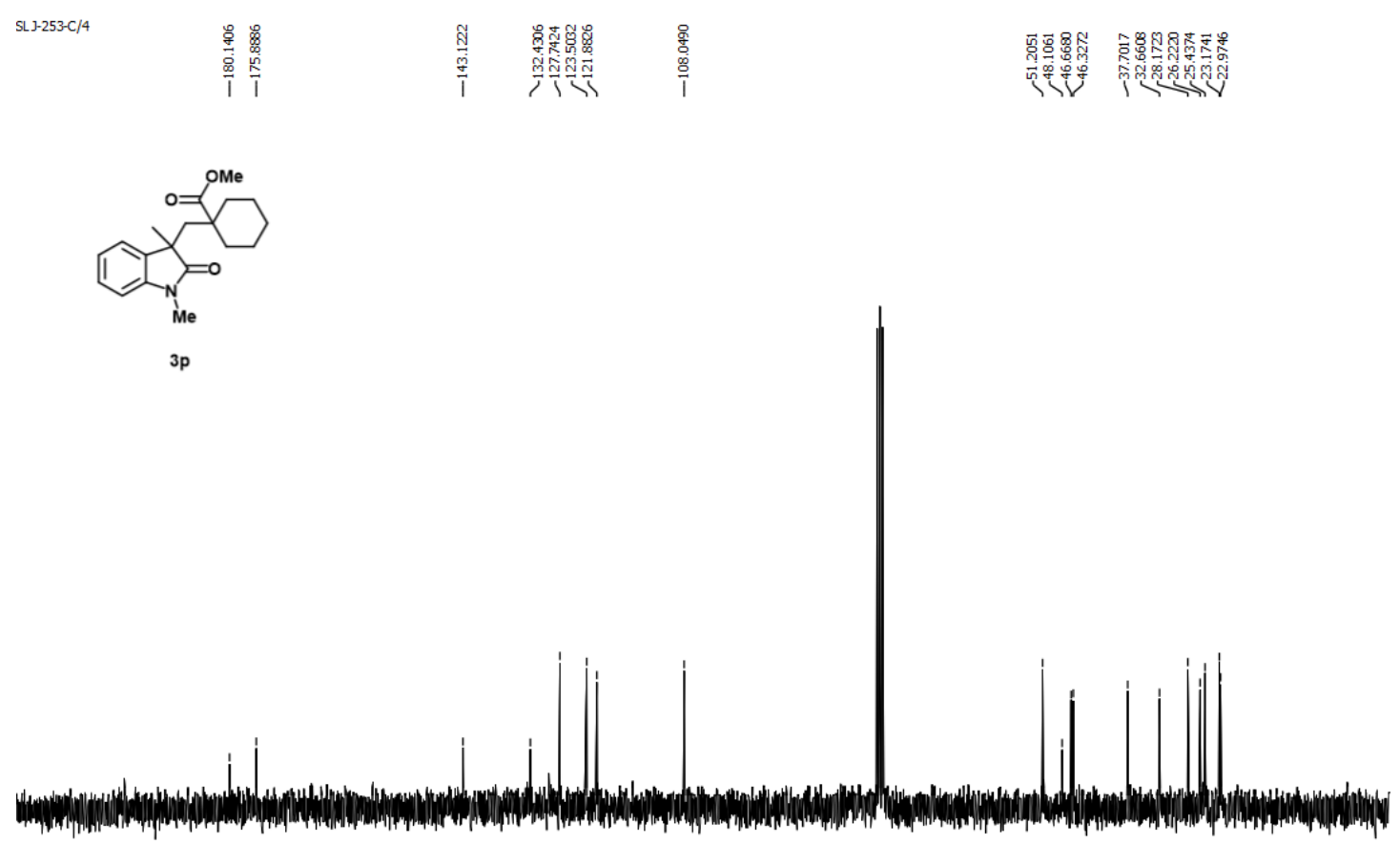

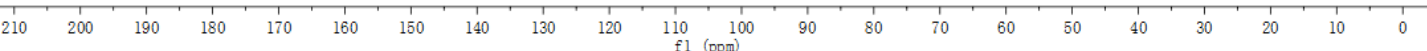


${ }^{1} \mathbf{H}$ NMR of compound $\mathbf{3 q}\left(300 \mathrm{MHz}\right.$ in $\left.\mathrm{CDCl}_{3}\right)$

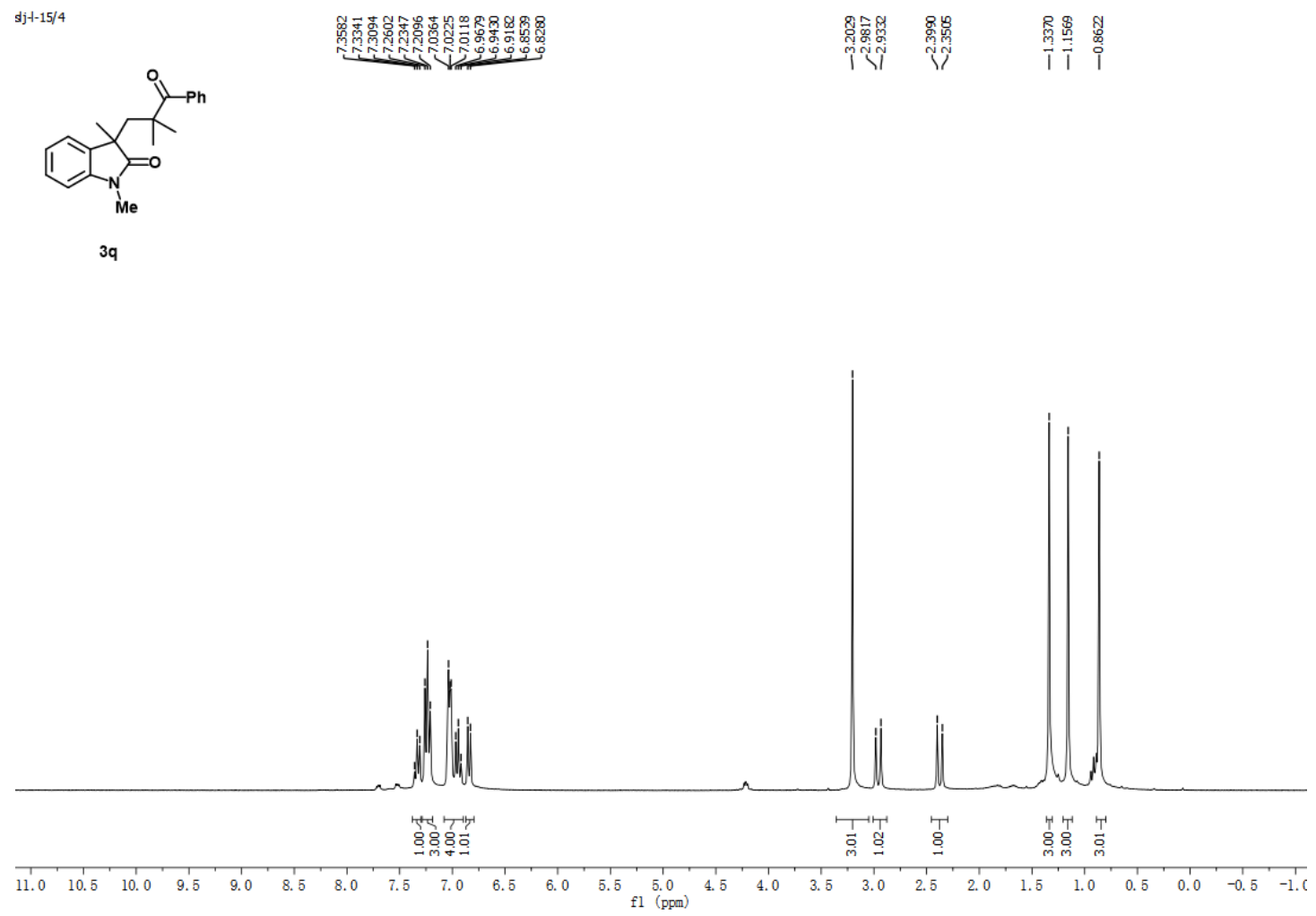

${ }^{13} \mathrm{C} \mathrm{NMR}$ of compound $\mathbf{3 q}\left(75 \mathrm{MHz}\right.$ in $\left.\mathrm{CDCl}_{3}\right)$

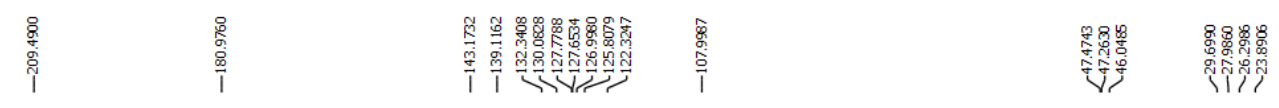

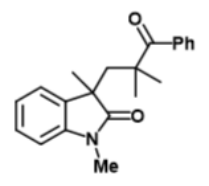

$3 q$

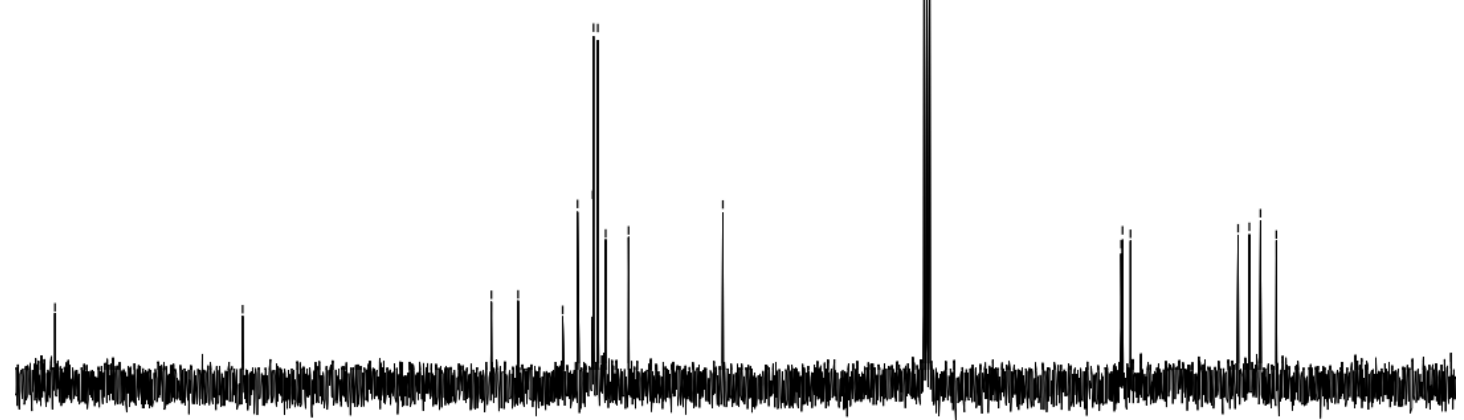

$\begin{array}{llllllllllllllllllllll}1 & 1 \\ 210 & 200 & 190 & 180 & 170 & 160 & 150 & 140 & 130 & 120 & \underset{f 1}{110}(\mathrm{ppm}) & 100 & 90 & 80 & 70 & 60 & 50 & 40 & 30 & 20 & 10 & 0\end{array}$ 
${ }^{1} \mathrm{H}$ NMR of compound $3 r\left(300 \mathrm{MHz}\right.$ in $\left.\mathrm{CDCl}_{3}\right)$

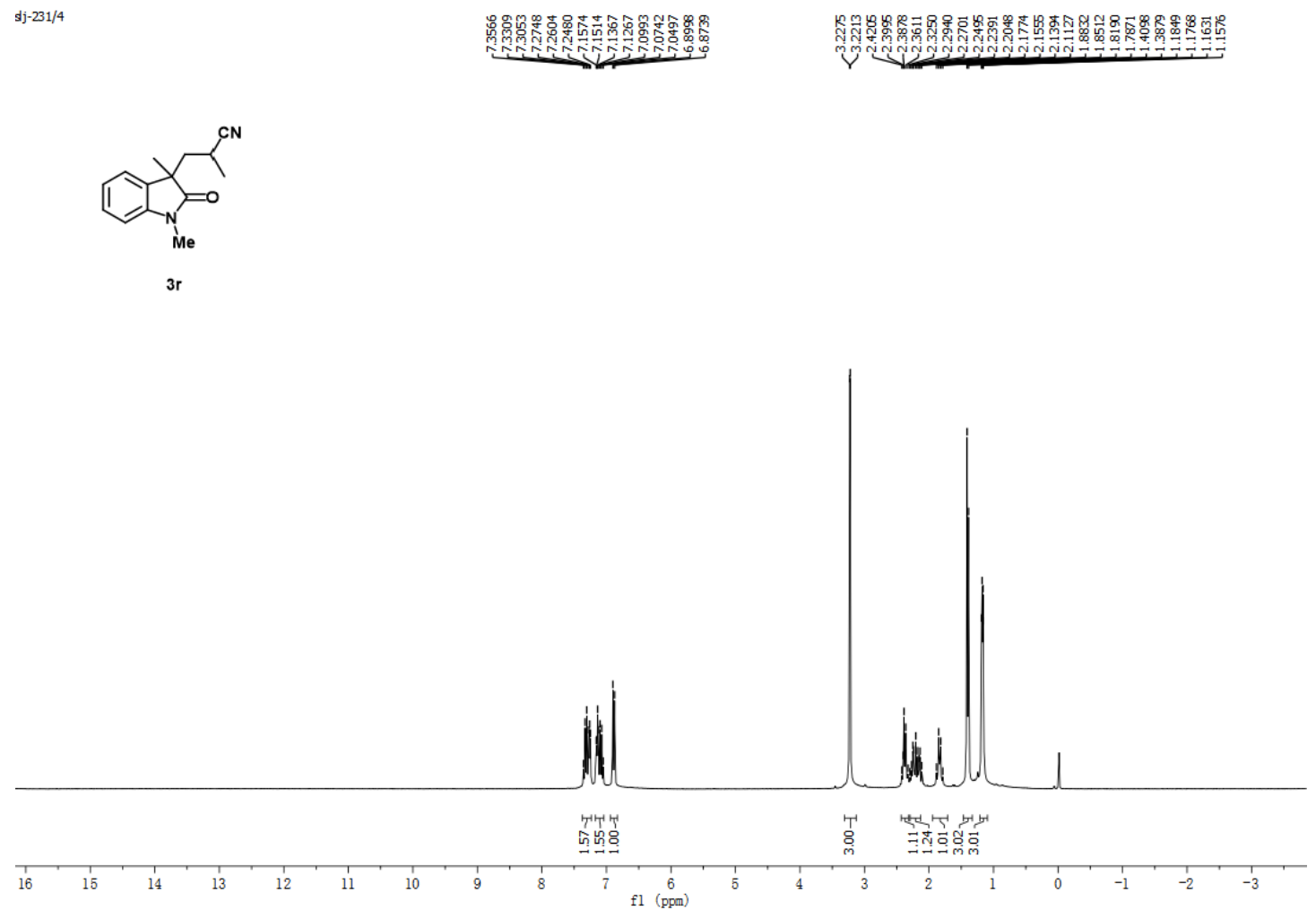

${ }^{13} \mathrm{C}$ NMR of compound $3 r\left(75 \mathrm{MHz}\right.$ in $\left.\mathrm{CDCl}_{3}\right)$

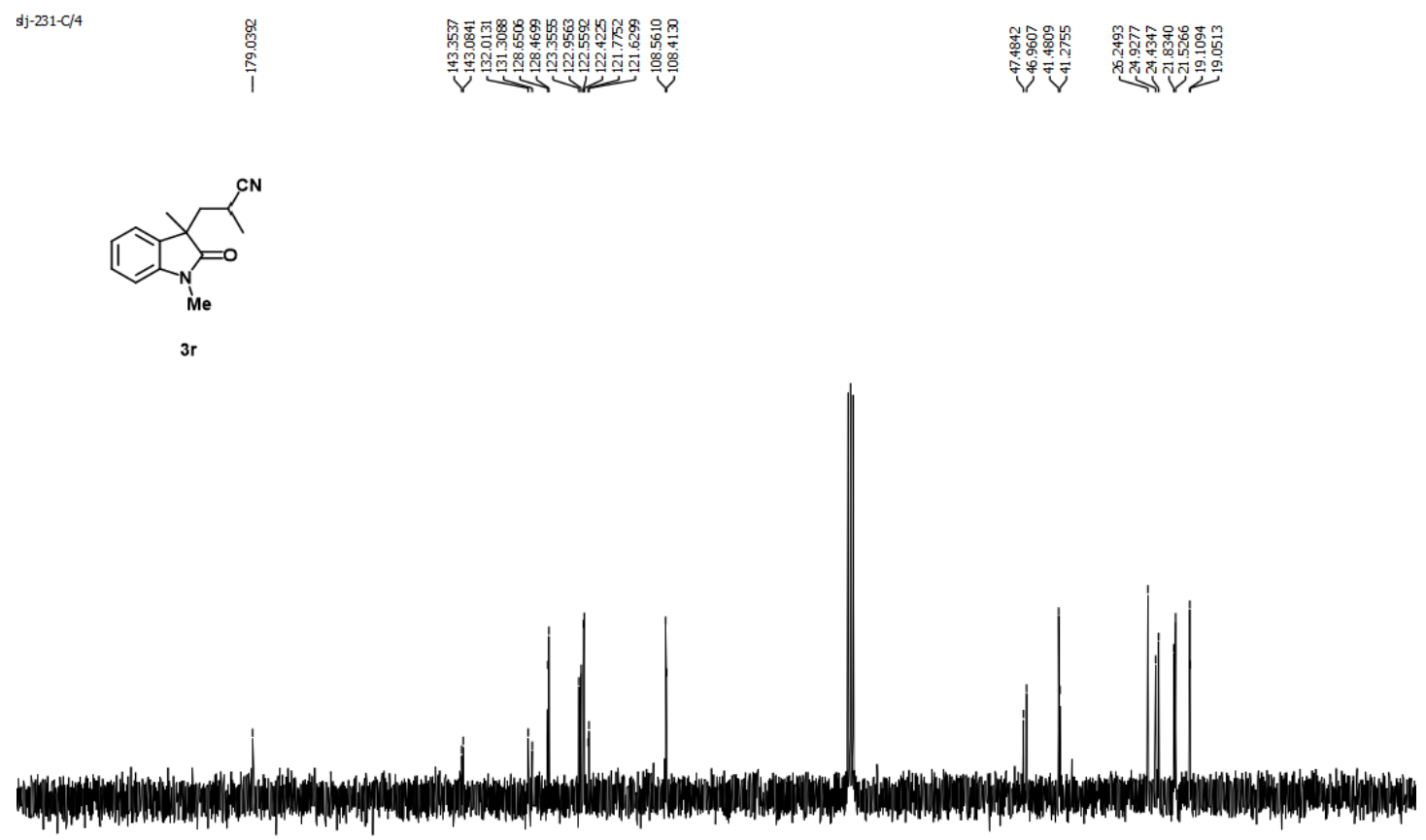

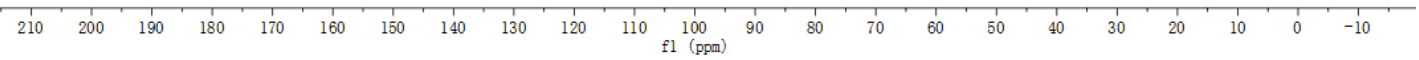


${ }^{1} \mathbf{H}$ NMR of compound $3 s\left(300 \mathrm{MHz}\right.$ in $\left.\mathrm{CDCl}_{3}\right)$

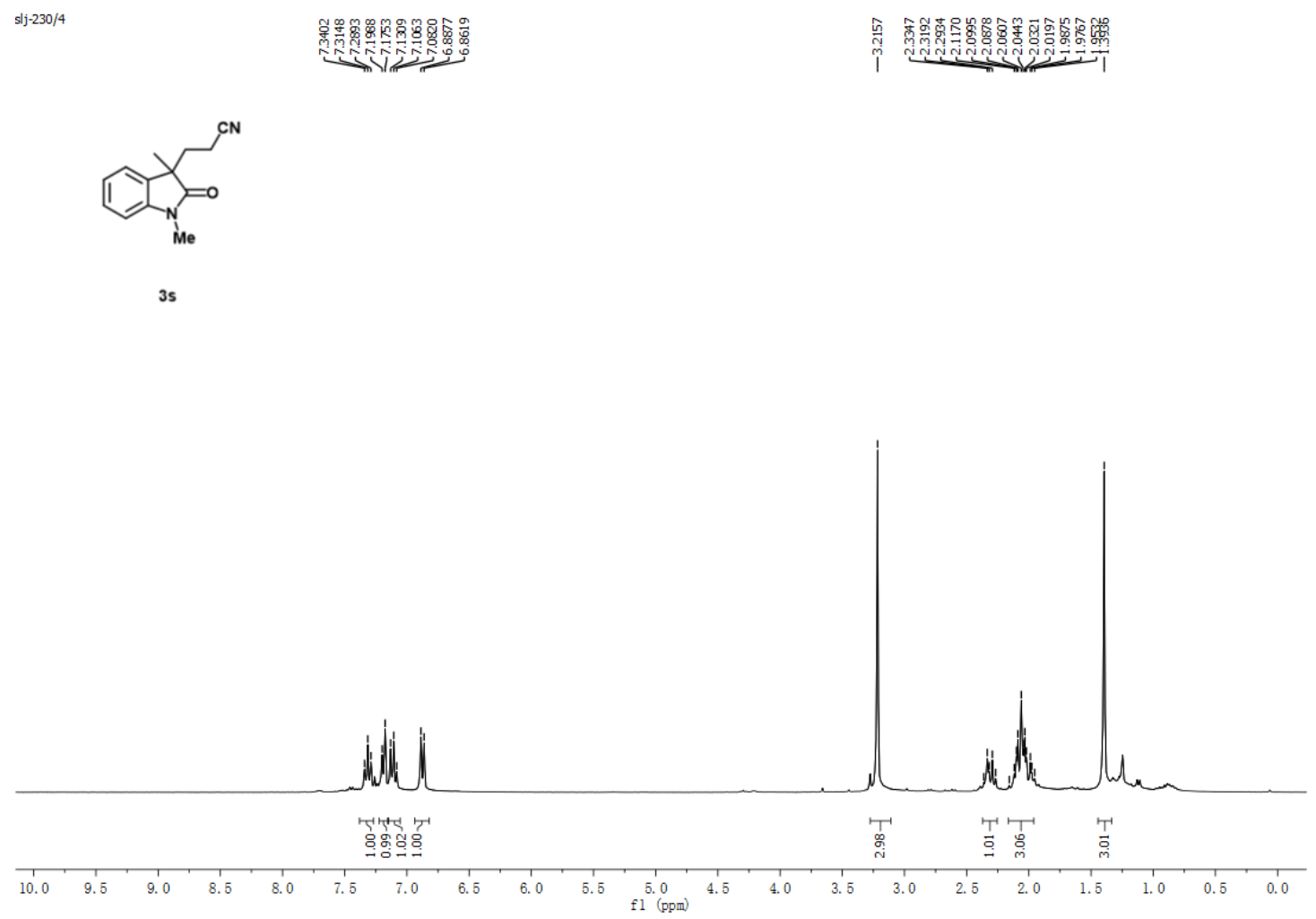

${ }^{13} \mathrm{C}$ NMR of compound 3s (75 $\mathrm{MHz}$ in $\left.\mathrm{CDCl}_{3}\right)$
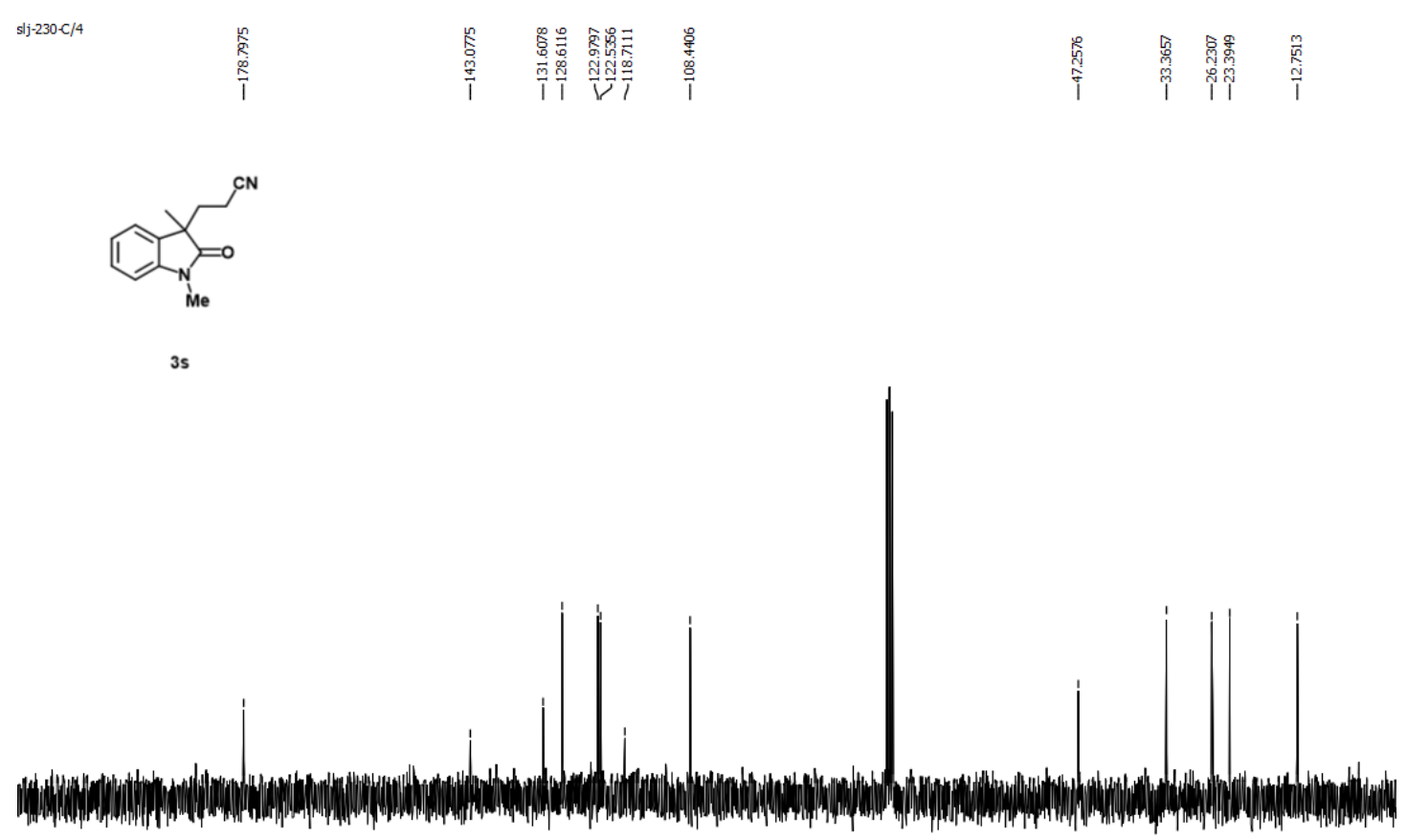

$\begin{array}{lllllllllll}210 & 200 & 190 & 180 & 170 & 160 & 150 & 140 & 130 & 120 & \begin{array}{l}110 \\ f 1\end{array}(\mathrm{ppm}) \\ 100 & \end{array}$ 
${ }^{1} \mathbf{H}$ NMR of compound $3 t\left(300 \mathrm{MHz}\right.$ in $\left.\mathrm{CDCl}_{3}\right)$

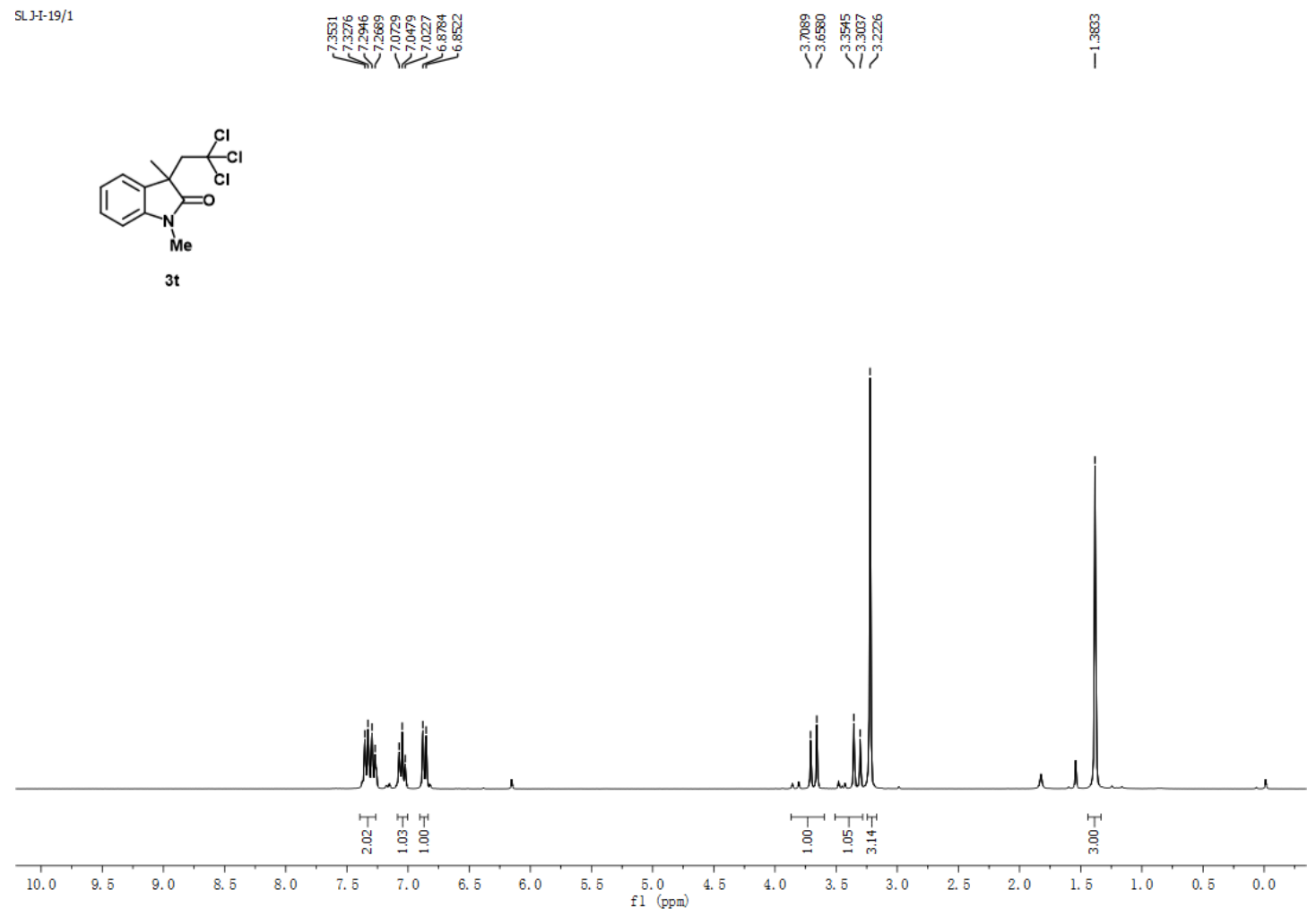

${ }^{13} \mathrm{C}$ NMR of compound $3 t\left(75 \mathrm{MHz}\right.$ in $\left.\mathrm{CDCl}_{3}\right)$
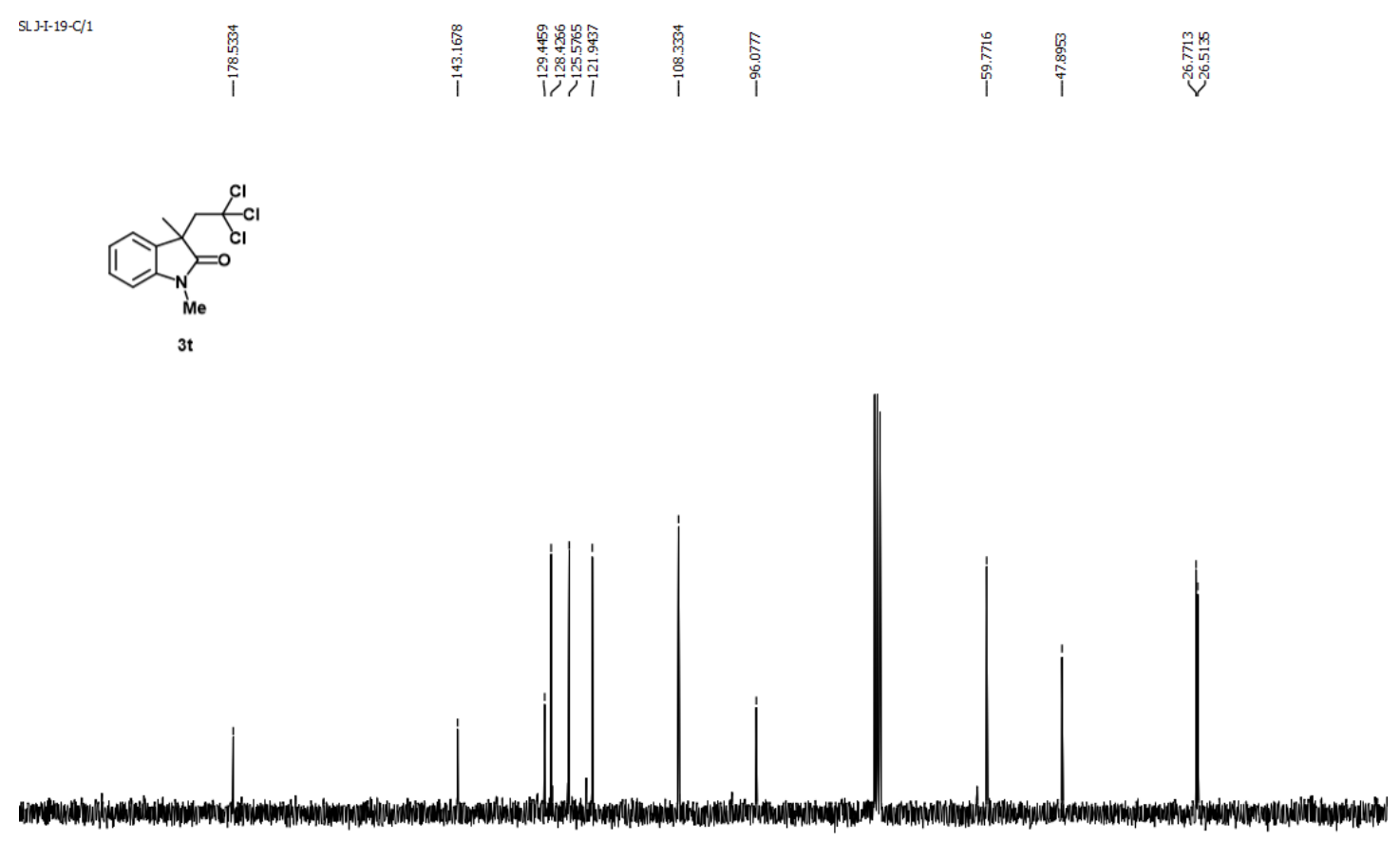

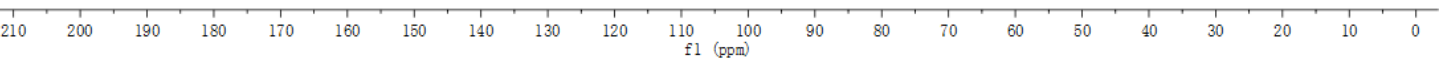


${ }^{1} \mathbf{H}$ NMR of compound $3 \mathbf{u}\left(300 \mathrm{MHz}\right.$ in $\left.\mathrm{CDCl}_{3}\right)$

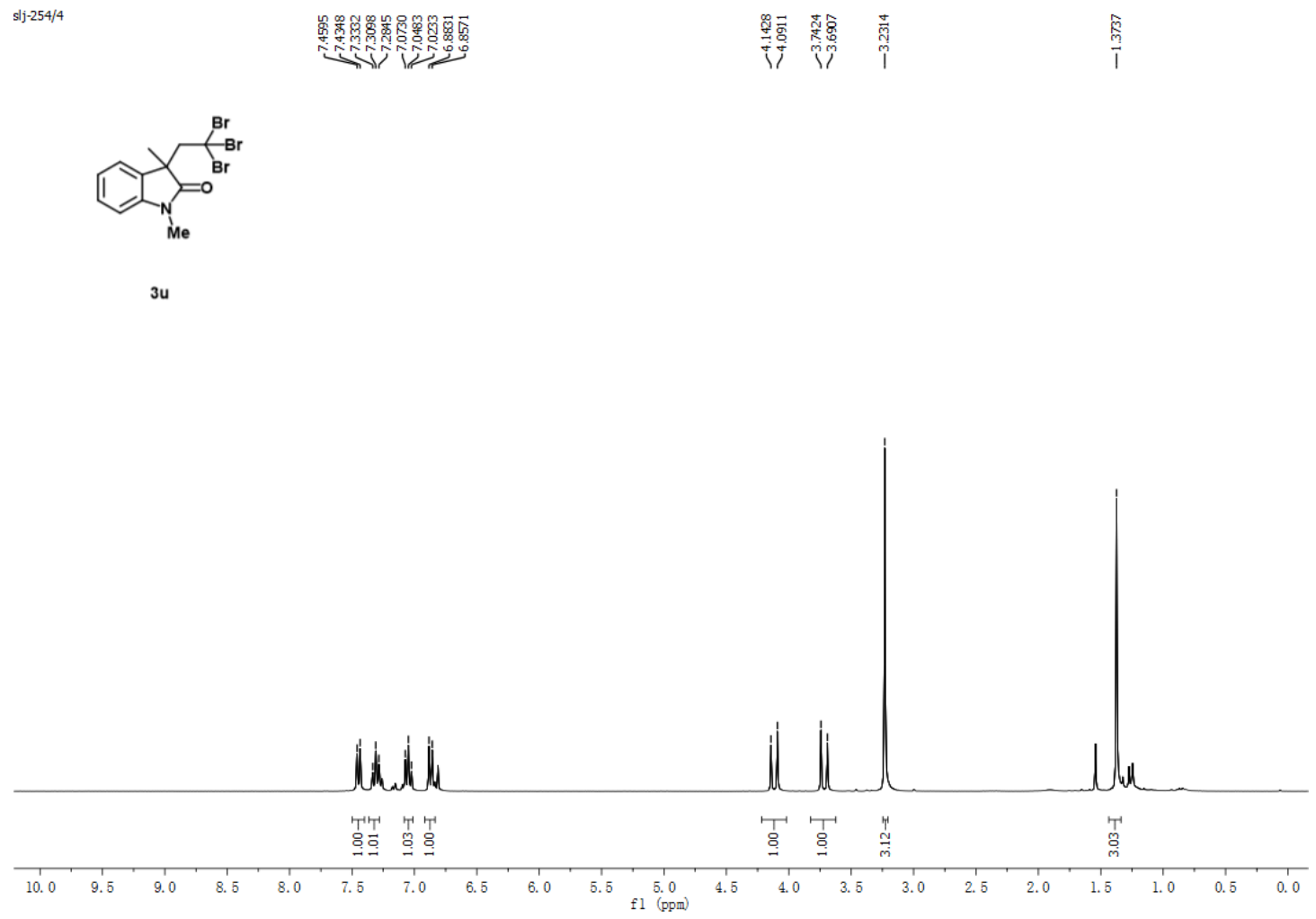

${ }^{13} \mathrm{C}$ NMR of compound $3 \mathrm{u}\left(75 \mathrm{MHz}\right.$ in $\left.\mathrm{CDCl}_{3}\right)$
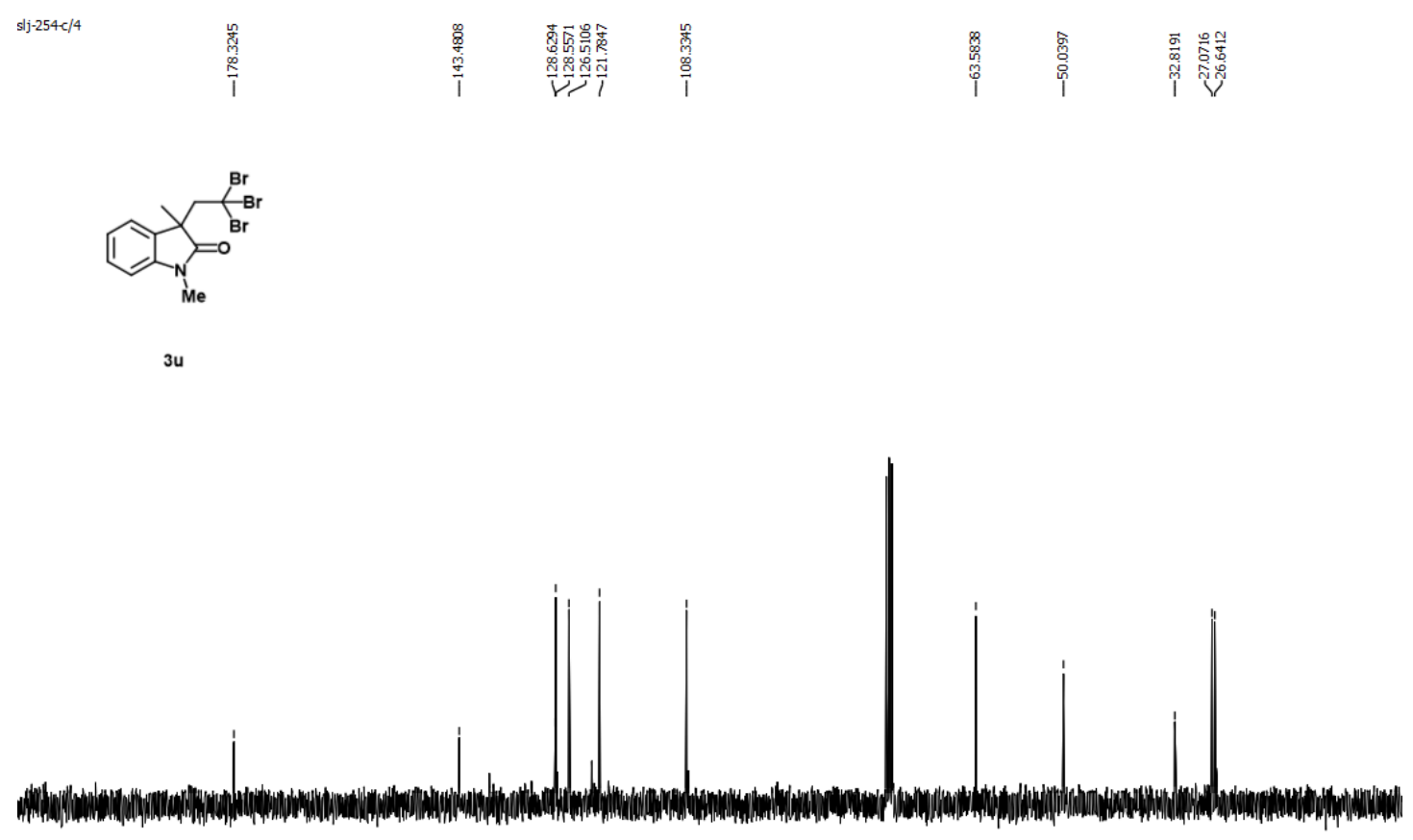

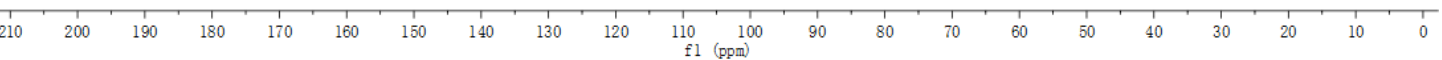


${ }^{1} \mathbf{H}$ NMR of compound $\mathbf{3} \mathbf{v}\left(300 \mathrm{MHz}\right.$ in $\left.\mathrm{CDCl}_{3}\right)$

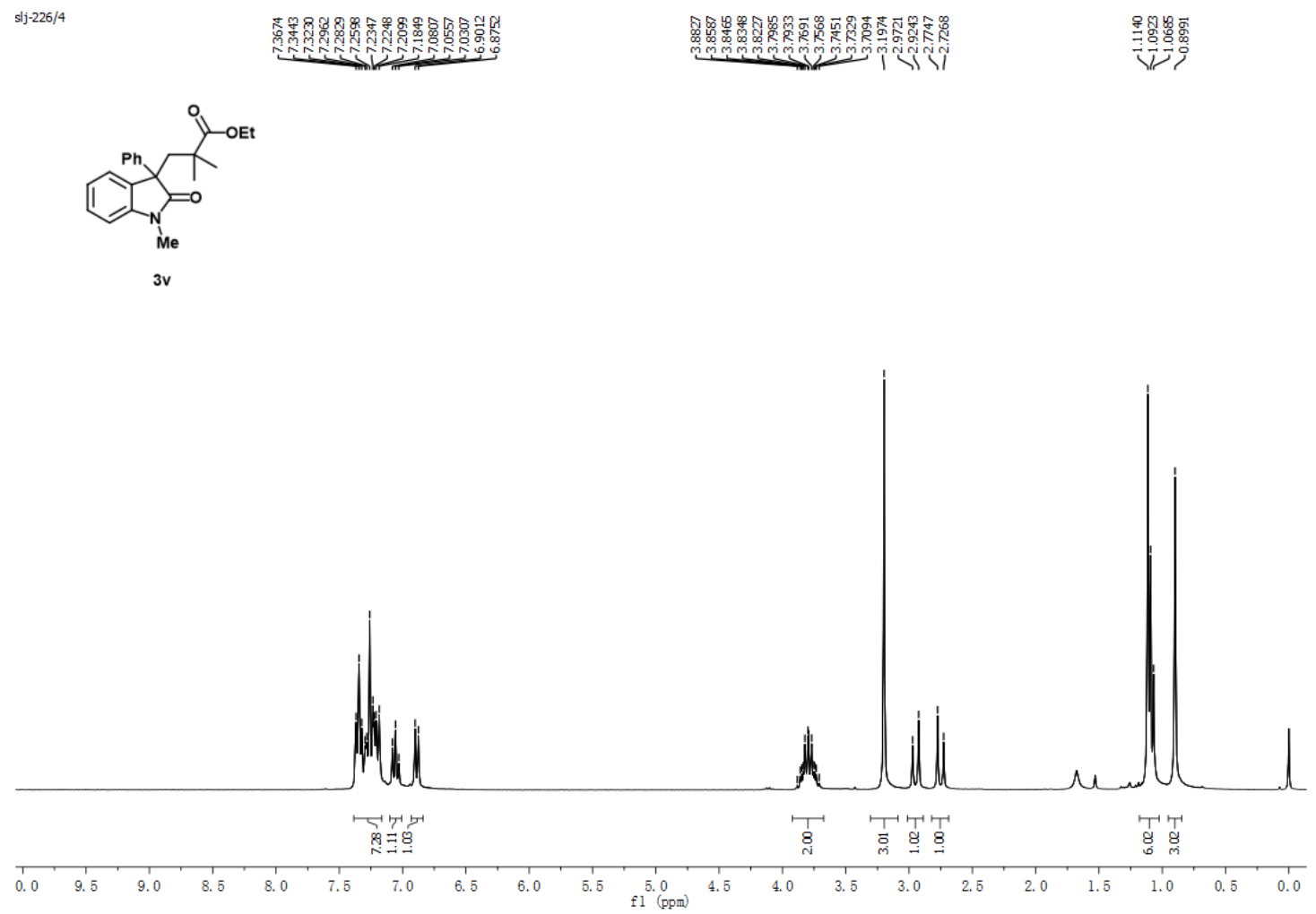

${ }^{13} \mathrm{C}$ NMR of compound $3 \mathbf{v}\left(75 \mathrm{MHz}\right.$ in $\left.\mathrm{CDCl}_{3}\right)$
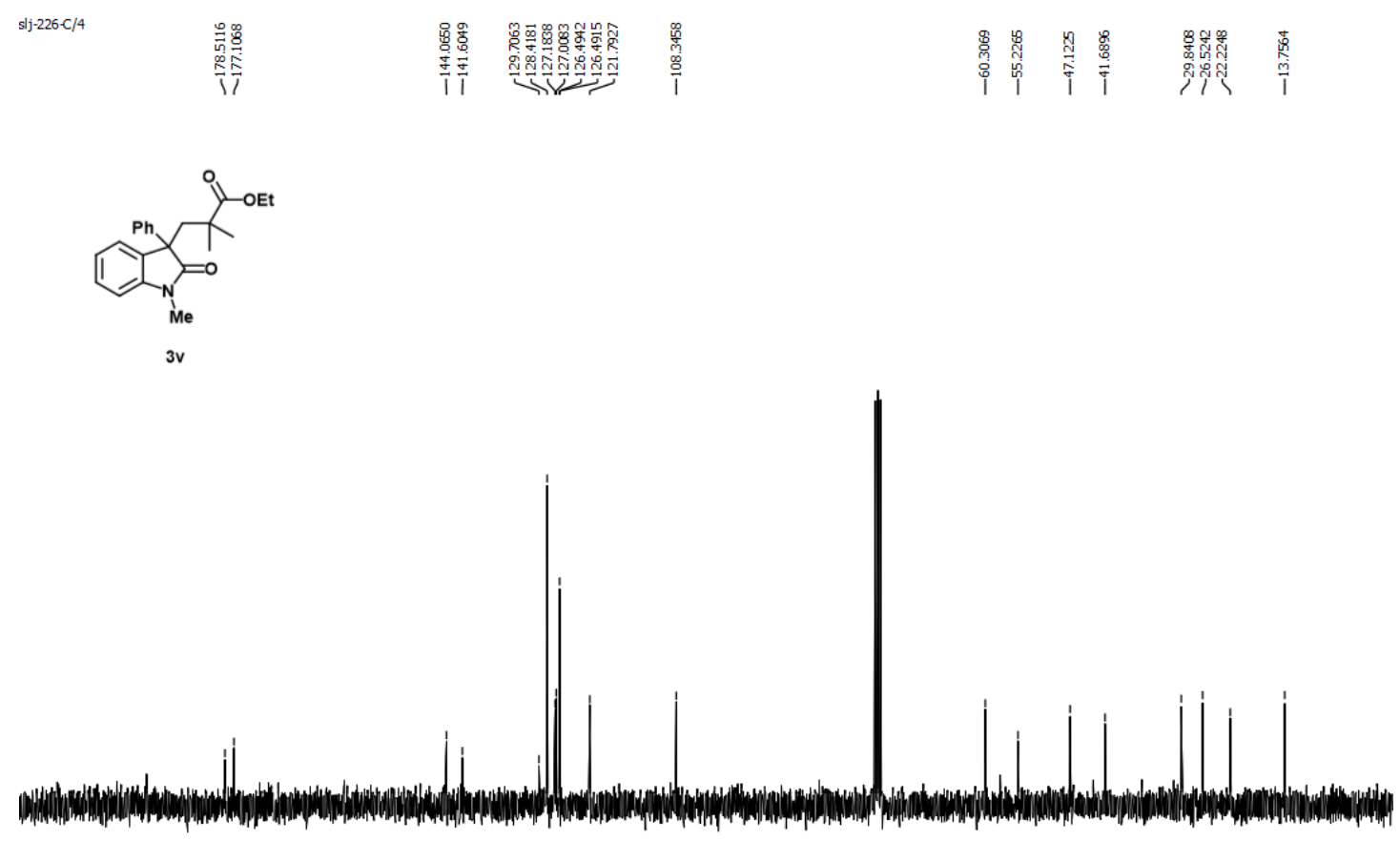

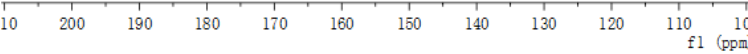


${ }^{1} \mathbf{H}$ NMR of compound $\mathbf{3 w}\left(300 \mathrm{MHz}\right.$ in $\mathrm{CDCl}_{3}$ )

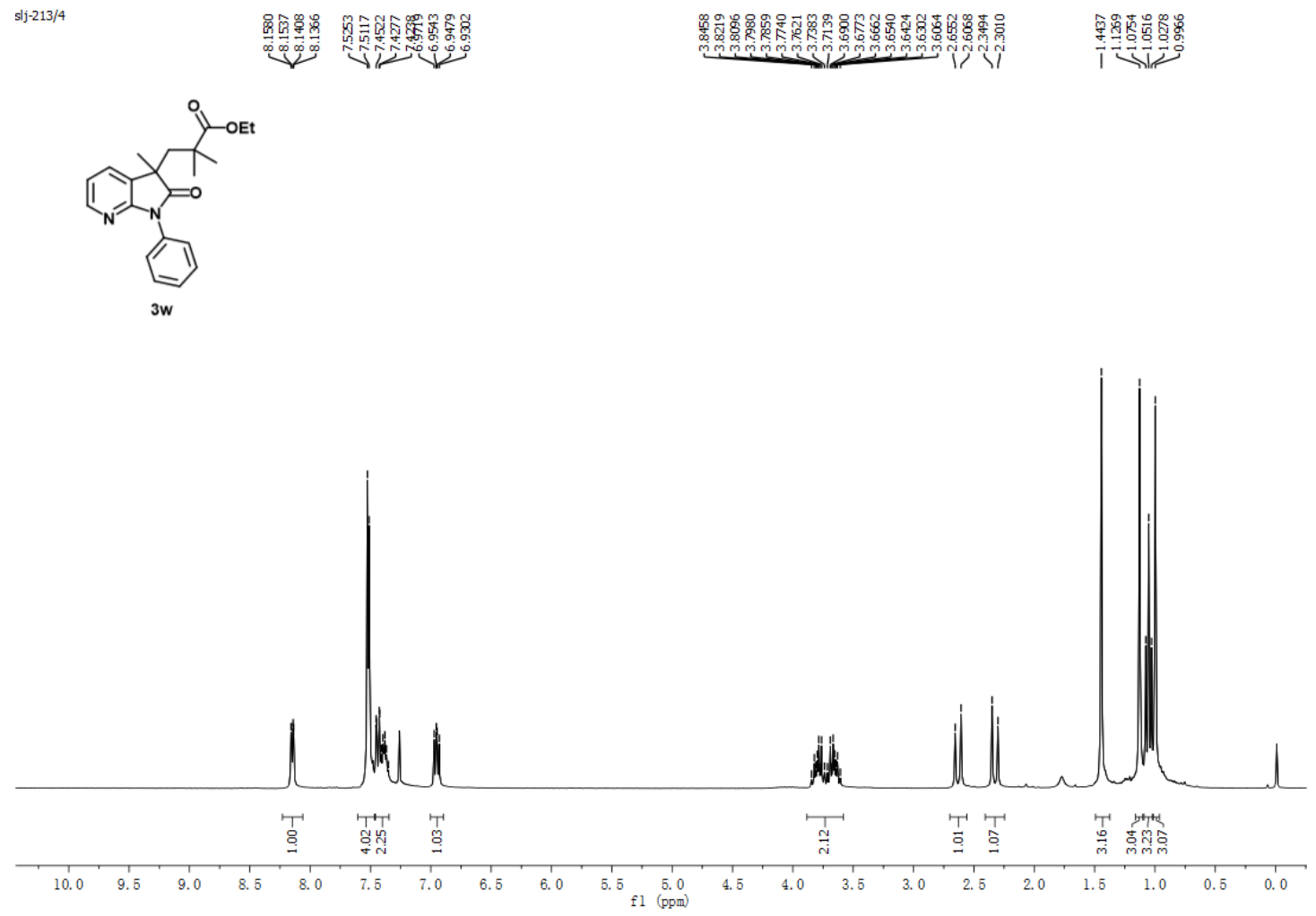

${ }^{13} \mathrm{C}$ NMR of compound $3 \mathbf{w}\left(75 \mathrm{MHz}\right.$ in $\left.\mathrm{CDCl}_{3}\right)$

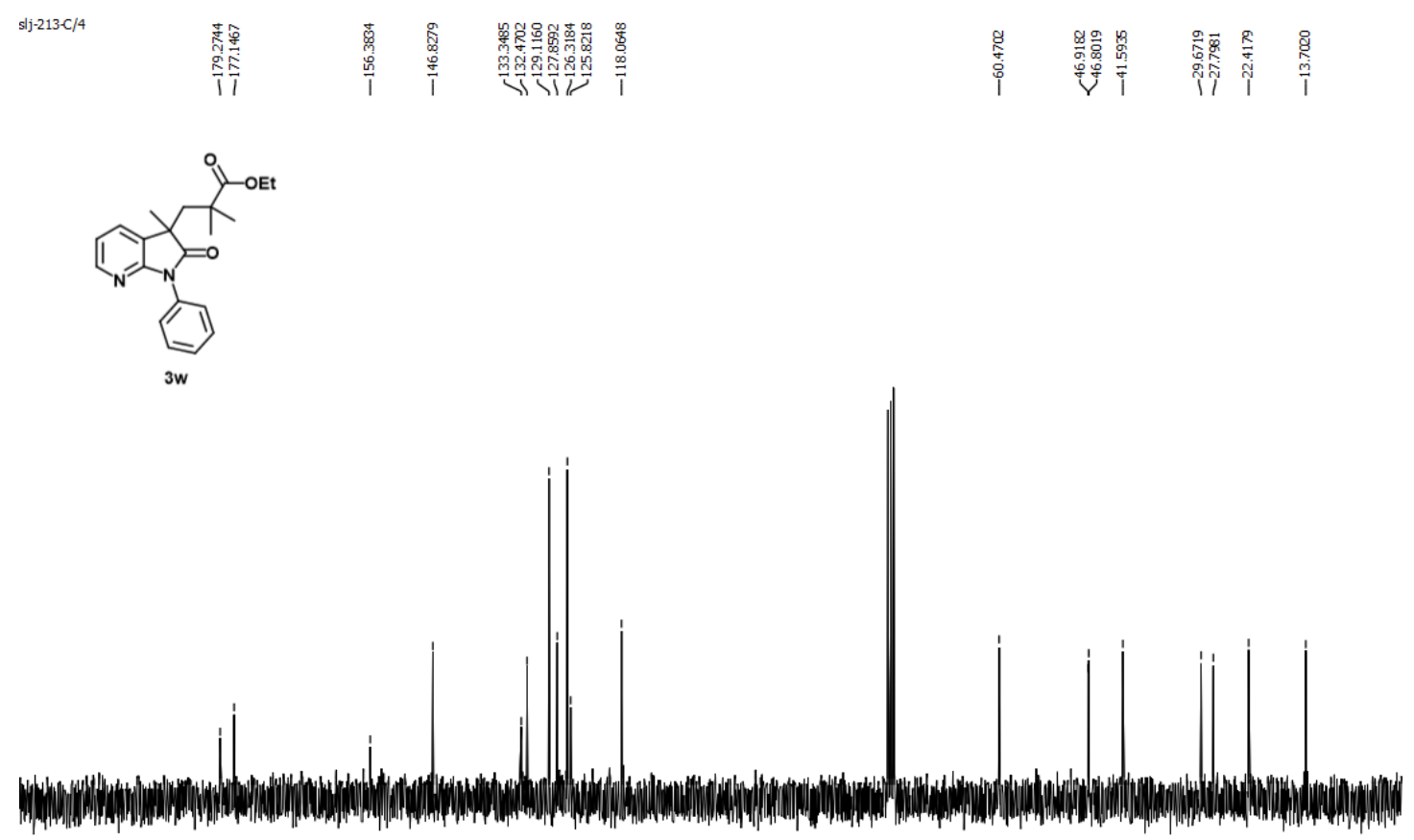

$\begin{array}{lllllllllll}200 & 190 & 180 & 170 & 160 & 150 & 140 & 130 & 120 & 110 & 100 \\ \mathrm{f} 1 & (\mathrm{ppm})\end{array}$ 
${ }^{1} \mathrm{H}$ NMR of compound $4 \mathrm{a}\left(300 \mathrm{MHz}\right.$ in $\left.\mathrm{CDCl}_{3}\right)$

s. $]-[-23-H / 1$

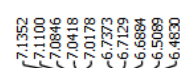

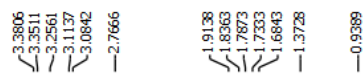

$\underbrace{\text { OH }}_{\substack{\text { ie } \\ 4 a}}$

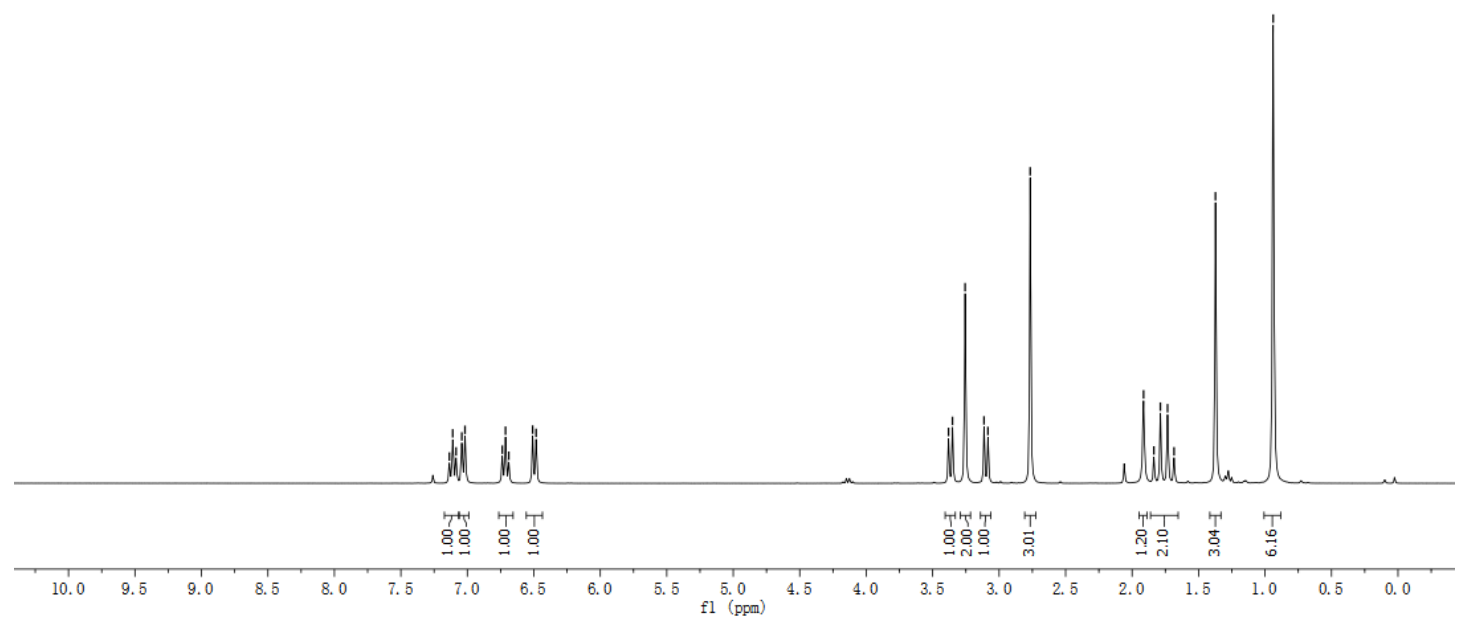

${ }^{13} \mathrm{C} \mathrm{NMR}$ of compound $4 \mathrm{a}\left(75 \mathrm{MHz}\right.$ in $\left.\mathrm{CDCl}_{3}\right)$ s.]-I-23-C/4

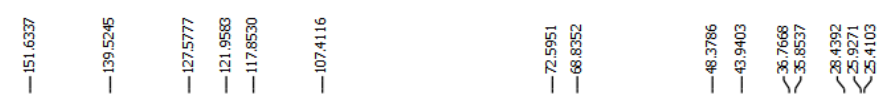<smiles>CCC1(CCC(C)(C)CO)Cc2ccccc2[C@H]1C</smiles>

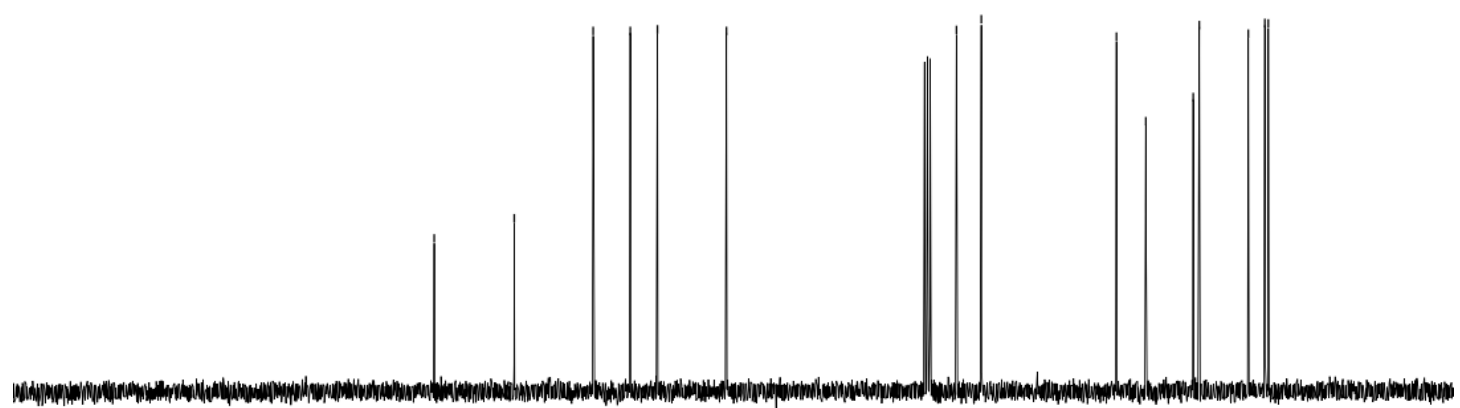

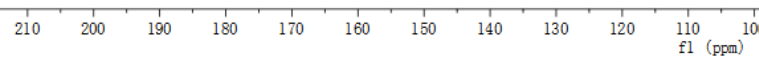


${ }^{1} \mathbf{H}$ NMR of compound $\mathbf{4 b}\left(300 \mathrm{MHz}\right.$ in $\left.\mathrm{CDCl}_{3}\right)$

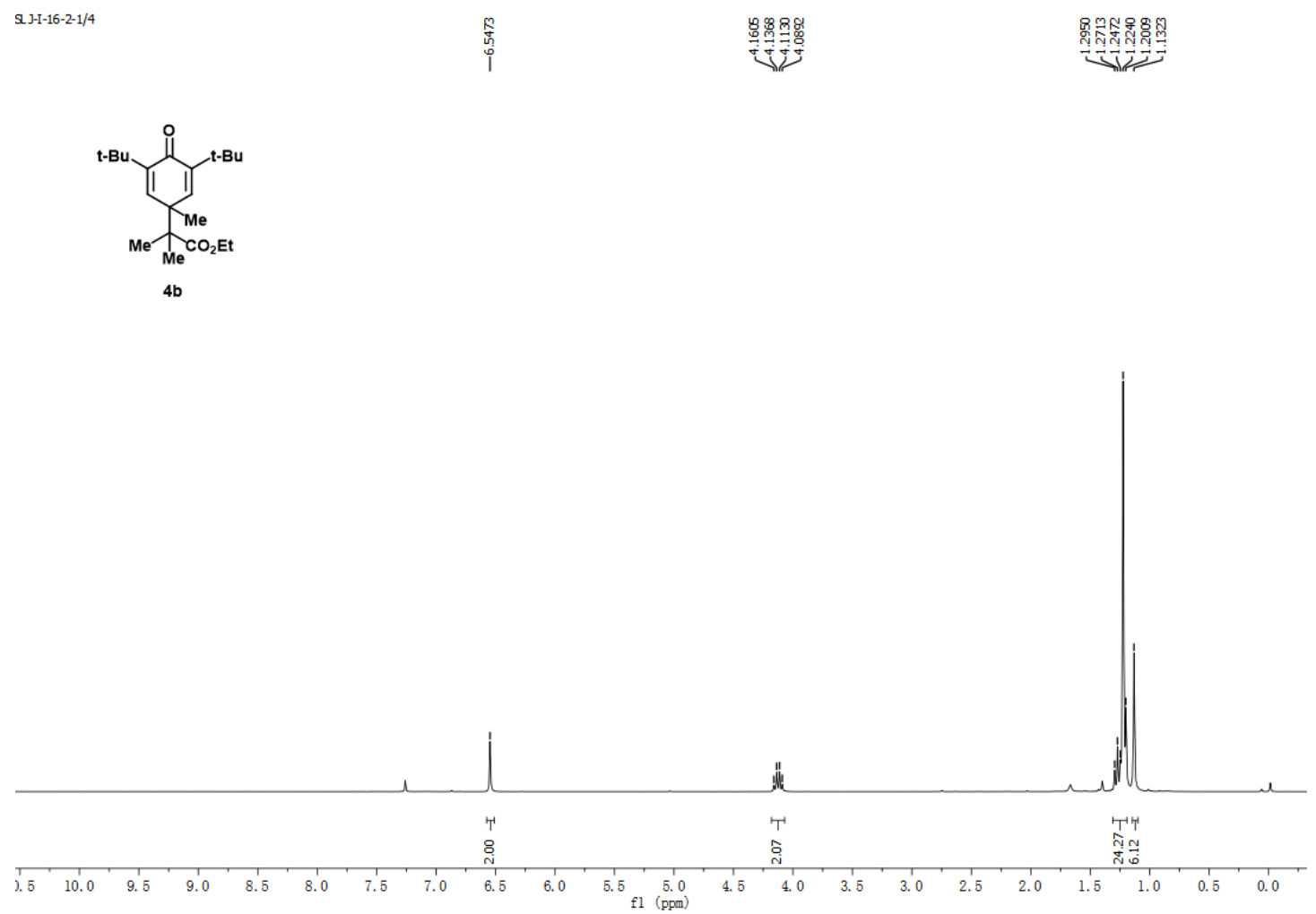

${ }^{13} \mathrm{C}$ NMR of compound $4 \mathrm{~b}\left(75 \mathrm{MHz}\right.$ in $\left.\mathrm{CDCl}_{3}\right)$

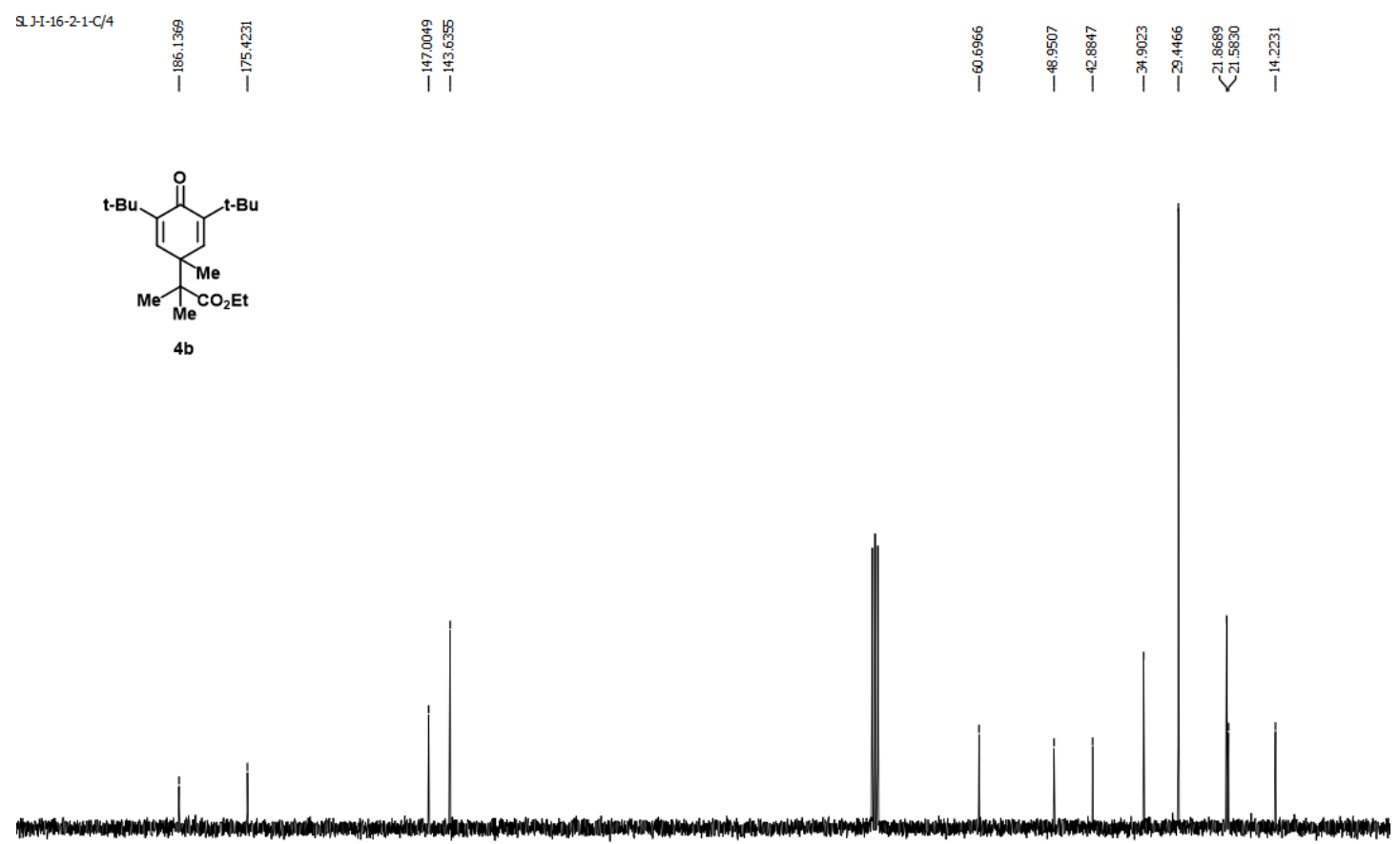

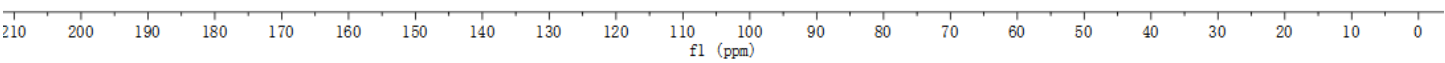

